\title{
ANTI-EPILEPTIC EFFECT OF LOW FREQUENCY STIMULATION USING THE KINDLING MODEL
}

\author{
by \\ Carys Alana Carrington, BSc (Hons)
}

\author{
A thesis submitted to \\ the Faculty of Graduate Studies and Research \\ In partial fulfillment of \\ the requirements for the degree of \\ Master of Science
}

Department of Psychology

Carleton University

Ottawa, Ontario

(c) Carys Alana Carrington, 2005 


$\begin{array}{ll}\begin{array}{l}\text { Library and } \\ \text { Archives Canada }\end{array} & \begin{array}{l}\text { Bibliothèque et } \\ \text { Archives Canada }\end{array} \\ \begin{array}{l}\text { Published Heritage } \\ \text { Branch }\end{array} & \begin{array}{l}\text { Direction du } \\ \text { Patrimoine de l'édition }\end{array} \\ \begin{array}{l}\text { 395 Wellington Street } \\ \text { Ottawa ON K1A 0N4 }\end{array} & \begin{array}{l}\text { 395, rue Wellington } \\ \text { Ottana ON K1A ON4 } \\ \text { Canada Oa }\end{array}\end{array}$

Your file Votre référence ISBN: 0-494-10132-6

Our file Notre référence

ISBN: 0-494-10132-6

NOTICE:

The author has granted a nonexclusive license allowing Library and Archives Canada to reproduce, publish, archive, preserve, conserve, communicate to the public by telecommunication or on the Internet, loan, distribute and sell theses worldwide, for commercial or noncommercial purposes, in microform, paper, electronic and/or any other formats.

The author retains copyright ownership and moral rights in this thesis. Neither the thesis nor substantial extracts from it may be printed or otherwise reproduced without the author's permission.
AVIS:

L'auteur a accordé une licence non exclusive permettant à la Bibliothèque et Archives Canada de reproduire, publier, archiver, sauvegarder, conserver, transmettre au public par télécommunication ou par l'Internet, prêter, distribuer et vendre des thèses partout dans le monde, à des fins commerciales ou autres, sur support microforme, papier, électronique et/ou autres formats.

L'auteur conserve la propriété du droit d'auteur et des droits moraux qui protège cette thèse. $\mathrm{Ni}$ la thèse ni des extraits substantiels de celle-ci ne doivent être imprimés ou autrement reproduits sans son autorisation.
In compliance with the Canadian

Privacy Act some supporting forms may have been removed from this thesis.

While these forms may be included in the document page count, their removal does not represent any loss of content from the thesis.
Conformément à la loi canadienne sur la protection de la vie privée, quelques formulaires secondaires ont été enlevés de cette thèse.

Bien que ces formulaires aient inclus dans la pagination, il n'y aura aucun contenu manquant. 


\begin{abstract}
It has been established that application of low frequency stimulation (LFS) to a kindled amygdala leads to the elevation of afterdischarge thresholds (ADTs) and thereby produces seizure and convulsion suppression effects. However, previous investigations have not been performed to determine if this is a focal effect, if it also occurs in the periphery and/or if there exists a genetic basis to the effects of LFS. The purpose of the study was twofold: 1) to examine the effects of LFS on ADTs in terms of its spatial characteristics; and 2) to address the possible genetic underpinnings of the LFS using genetically predisposed (FAST) and resistant (SLOW) strains of rats.

First, the animals were kindled in the amygdala and the low frequency manipulations were carried out to determine their effects on the amygdala ADTs and ensuing convulsive seizures through a twisted, discrete kindling electrode in the amygdala versus a spanning electrode, covering a broader region in the temporal lobe. LFSs of the normal electrode produced an elevation of the ADTs in both rat strains, which abated over a 4-day period. A much smaller effect on the ADTs was observed after the application of LFS to the spanning electrode. It was also found that although an initially longer duration stimulus augments the effect of the LFS, with a longer duration stimulus (300s) a "mild-status" state, with spontaneously occurring seizures was generated. The significance of this finding was that this form of status epilepticus lacked the pathology commonly observed in the other animal models of this condition and whose pathophysiology did not depend upon a kindled brain to be expressed.
\end{abstract}




\section{ACKNOWLEDGEMENTS}

My deepest appreciation and gratitude I offer to my supervisor, Dr. Dan McIntyre who has, over the years, been a great source of support and encouragement. I am awed by his passionate commitment to epilepsy research and am eternally grateful to have had the honor of working with such an esteemed scholar. To Dr. Krista Gilby: thank you for being there when I needed help the most and for your honesty in telling me what I needed to hear.

I give my thanks to the many amazing people who have supported me along the way, my LSRB buddies: Rick, Priya, Natalie, Sabrina, Cezar, Jennifer, Pete, Tina and especially Kathryn. To Laura Cohen who has been like a big sister to me in more ways than one. To Alvin Mallon, who kept a starving student well fed when stuck at the library or lab at godforsaken hours, for maintaining a necessary supply of chocolate and for caring, I thank you. Also, to all the staff at the MacOdrum Library who have encouraged me along the way, especially Gilles, Michelle and Marsha. Etelle and Nathalie, thank you for answering my many questions, allaying my fears and for dealing patiently with my many letter requests.

Special thanks to two very special people: Pavel, from the bottom of my heart I thank you for encouraging me on when I thought that I would fail; for being honest even when I didn't want to hear the truth and for seeing in me what I could not see for myself. Emdera, thank you for opening your home and heart to me, for making sure I didn't leave the house without breakfast and for always being supportive.

My warmest thanks to my family, my aunt and cousin who have opened up their home and lives to me over the many years it took me to get this far. To my sisters, brother and father, I thank you for your timeless support and sacrifices that you made for me over the years. Finally, I dedicate this thesis to my mother, your boundless, unconditional love and support has been my mainstay, guiding me along this path that I have chosen. If I could be a mere fraction of the person you are I would be blessed. I love you.

To those who I have not mentioned by name, and there are many, I thank you all. 


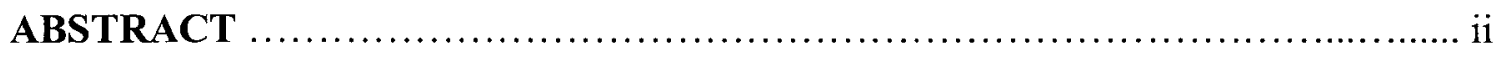

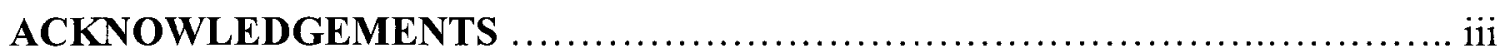

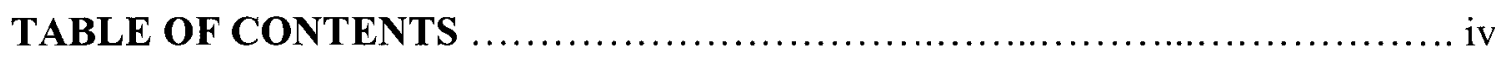

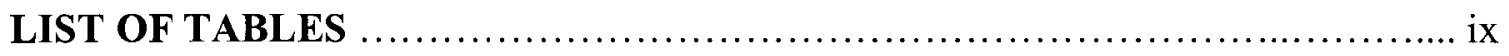

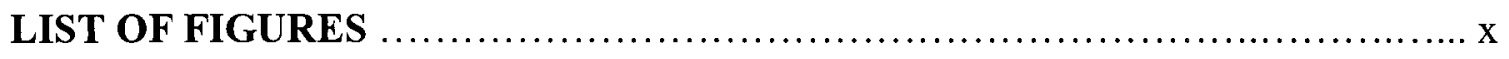

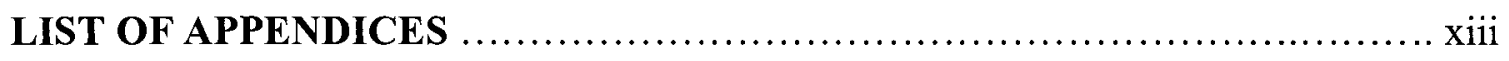

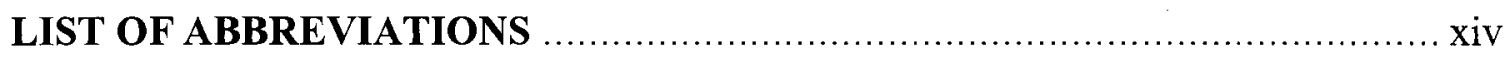

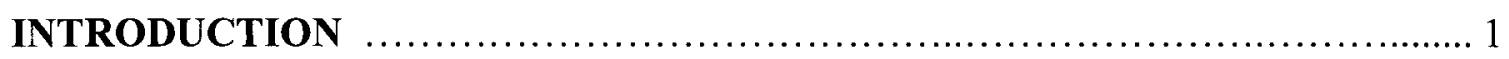

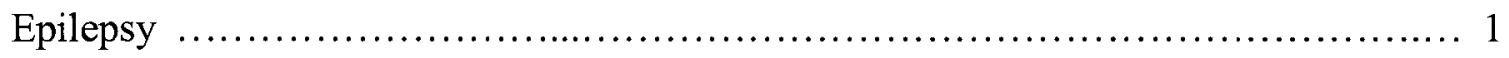

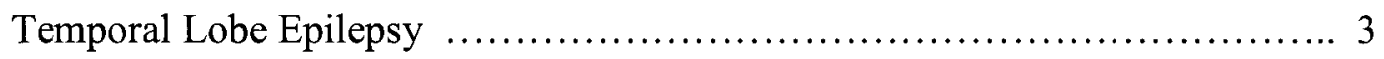

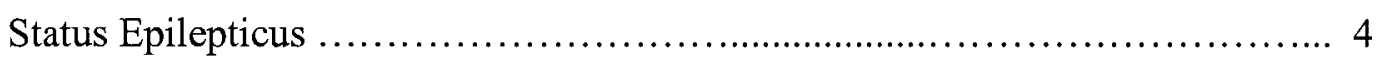

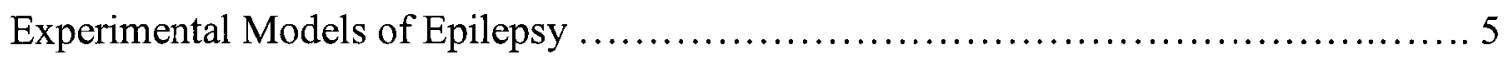

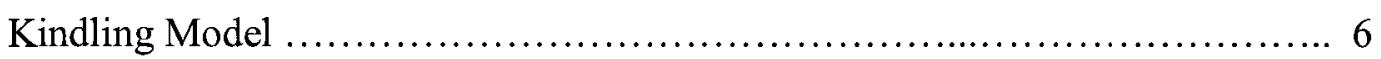

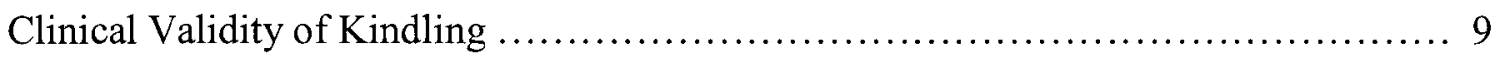

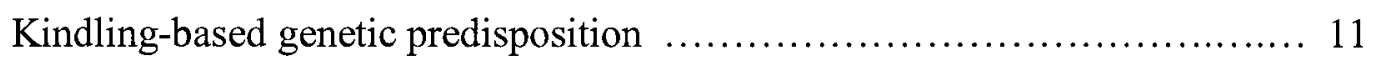

Electrical Stimulation in the Epilepsies ....................................... 14

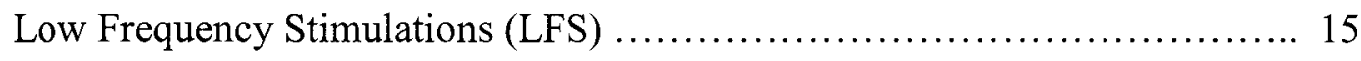

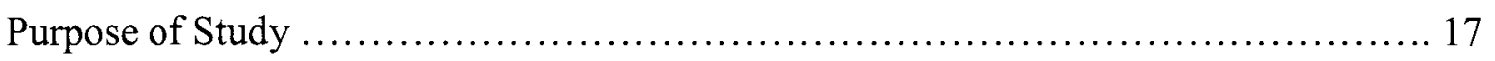

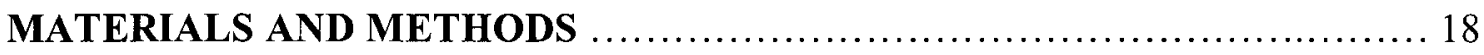

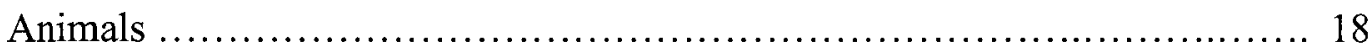

EXPERIMENT 1: Kindling and Stability of After Discharge

Thresholds (ADTs) $\ldots \ldots \ldots \ldots \ldots \ldots \ldots \ldots \ldots \ldots \ldots \ldots \ldots$ 
Animals 18

Surgery 18

Kindling and Afterdischarge thresholds (ADTs). 24

Low Frequency Stimulation (LFS) Manipulations 25

1) LFS administered to kindled left amygdala with ADT tested on kindled left amygdala

2) LFS administered to non-kindled left spanning area with ADT tested on kindled left amygdala. 26

3) LFS administered to kindled left spanning area with ADT tested on kindled left spanning area

4) LFS administered to kindled left spanning area with $A D T$ tested on previously kindled left amygdala 26

Histology 27

Statistical Analysis 27

RESULTS 28

Kindling Rates and Afterdischarge Thresholds (ADTs) 28

Low Frequency Stimulation (LFS) Manipulations 37

1) LFS administered to kindled left amygdala with ADT tested on kindled left amygdala 37

2) LFS administered to non-kindled left spanning area with ADT tested on kindled left amygdala.... 40

3) LFS administered to kindled left spanning area with ADT tested on kindled left spanning area 43 
4) LFS administered to kindled left spanning area with ADT tested on previously kindled left amygdala ..................... 43

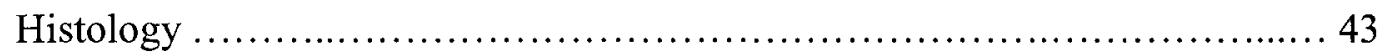

EXPERIMENT 2: Low Frequency Stimulation Applied Contralaterally ........ 46

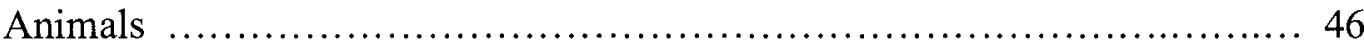

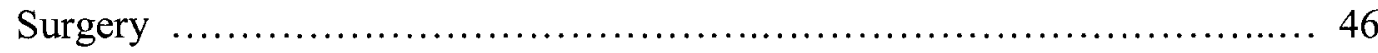

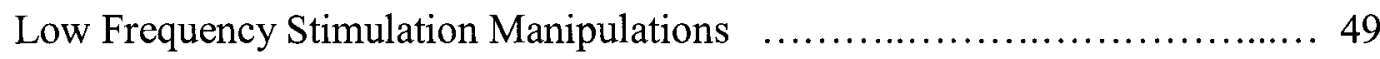

Kindled right discrete amygdala electrode (Group 1) ................ 49

Kindled right discrete amygdala electrode (Group 1) .............. 50

Histology and Data Analysis .................................... 50

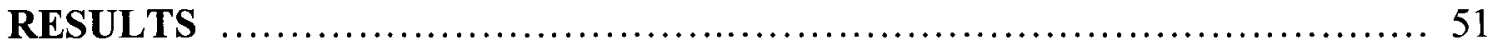

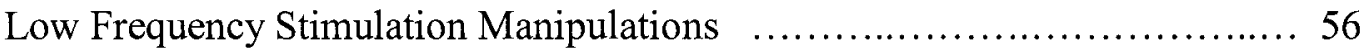

Kindled right discrete amygdala electrode (Group 1) ................ 56

Kindled right discrete amygdala electrode (Group 1) ...............6 60

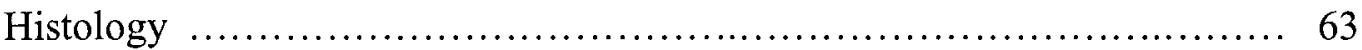

EXPERIMENT 3: Effect of Altering Low Frequency Stimulation Parameters ... 64

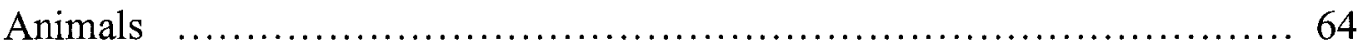

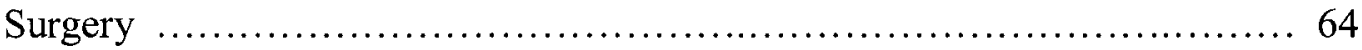

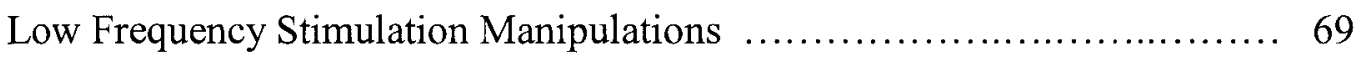

Low Frequency Stimulation - intensity and duration manipulations ... 69

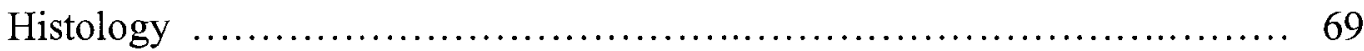

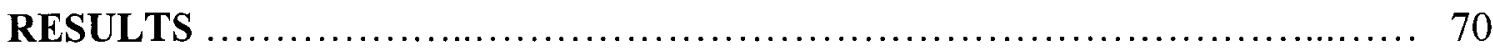

Low frequency stimulation at $100 \mu \mathrm{A}$ administered to discrete and 
spanning electrodes

Low frequency stimulation at varying intensities administered to the spanning

electrode 74

Low frequency stimulation at long duration and moderate intensity 74

Low frequency stimulation and mild-status effect.. 75

Low frequency stimulation and seizure suppression. 75

Histology 80

EXPERIMENT 4: Triggering Spontaneous Seizures with Low Frequency

Stimulation 83

Animals 83

Surgery 83

Low Frequency Stimulation (LFS) Manipulations 84

Histology 84

RESULTS 84

Low Frequency Stimulation Manipulations 84

1) LFS administered to kindled right amygdala with ADT tested on kindled right amygdala 84

2) LFS administered to non-kindled left spanning electrode with ADT tested on kindled right amygdala... 88

Histology 93

EXPERIMENT 5: Kindled Brain and the Pseudo-Status Effect 94

Animals 94

Surgery and Kindling 94 
Histology 95

Low Frequency Stimulation (LFS) Manipulations 95

RESULTS 95

DISCUSSION 98

CONCLUSION 108

APPENDIX 109

REFERENCES .110 


\section{LIST OF TABLES}

TABLE DESCRIPTION

PAGE

1

Average ( \pm S.E.M.) percent of Fast and Slow .91 rats that experienced spontaneous seizures, seizure failures and immeasurable ADTs after the application of LFS on the kindled amygdala and spanning electrode with ADTs tested on the kindled amygdala.

2

Average ( \pm S.E.M.) percent of Kindled and...............96 Non-kindled rats that experienced spontaneous seizures and seizure failures. 


\section{LIST OF FIGURES}

Figure 1. Configuration of the "traditional" twisted, discrete electrode and spanning electrode.

Figure 2. Schematic representation, (Paxinos and Watson, 2005) of electrode placement showing targeted regions.

Figure 3. Average ( \pm S.E.M.) number of stimulations required to elicit the first stage 5 seizure (i.e. kindling rate) for the FAST and SLOW strains as a function of the kindling site.

Figure 4. Average ( \pm S.E.M.) kindling rate for the discrete (amygdala) electrode and the spanning electrode

Figure 5. Average ( \pm S.E.M.) percent change in the ADTs in the days following the establishment of the kindled state.

Figure 6. a) Average ( \pm S.E.M.) percent change in the ADTs in the time following application of LFS $(1 \mathrm{~Hz} ; 100 \mu \mathrm{A} ; 30 \mathrm{~s})$ to the kindled discrete amygdala with $\mathrm{ADT}$ measured from the kindled amygdala electrode

b) Average ( \pm S.E.M.) percent change in the ADTs in the time following application of LFS $(1 \mathrm{~Hz} ; 100 \mu \mathrm{A} ; 90 \mathrm{~s})$ to the kindled discrete amygdala with ADTs measured from the kindled amygdala electrode.

Figure 7. Average ( \pm S.E.M.) percent change in afterdischarge thresholds (ADTs) Following the application of the LFS (1 Hz; 100 $\mu \mathrm{A} ; 30 \mathrm{~s})$ to the spanning electrode in the left amygdala with ADTs measured from the kindled amygdala electrode.

Figure 8. A photomicrograph of the left basolateral amygdala of a FAST rat (Carys \#4) stained with Cresyl Violet showing necrosis around the point of insertion of a discrete electrode (Leica Fluorescent Microscope, magnification 10X).

Figure 9. Schematic representation, (Paxinos and Watson, 2005) of the electrode placements showing the targeted regions.

Figure 10. Average ( \pm S.E.M.) number of stimulations required to elicit the initial stage 5 seizure in the FAST and SLOW strains as a function of the kindling site (the right discrete amygdala vs. the left spanning region). 
Figure 11. Average ( \pm S.E.M.) percent change of ADTs of the FAST vs. the SLOW rats following the establishment of the kindled state through the right amygdala discrete electrode and the left spanning electrode.........................54

Figure 12. a) Average ( \pm S.E.M.) percent change in ADT in the time following application of a LFS $(1 \mathrm{~Hz} ; 100 \mu \mathrm{A} ; 30 \mathrm{~s})$ to the kindled amygdala with ADTs measured from the kindled discrete amygdala electrode.

b) Average ( \pm S.E.M.) percent change in ADTs in the time following application of a LFS $(1 \mathrm{~Hz} ; 100 \mu \mathrm{A} ; 90 \mathrm{~s})$ to the kindled amygdala with ADTs measured from the kindled discrete amygdala electrode.

Figure 13. Average ( \pm S.E.M.) percent change in the ADTs in the time following the application of LFS $(1 \mathrm{~Hz} ; 100 \mu \mathrm{A})$ at $30 \mathrm{sec}$ and $90 \mathrm{sec}$ durations to the kindled spanning electrode with the ADTs measured from the same electrode.

Figure 14. Schematic representation, (Paxinos and Watson, 2005) of the electrode placements showing targeted regions.

Figure 15. Schematic representation of the modified spanning electrode.

Figure 16. Average ( \pm S.E.M.) percent change ADTs in the time following

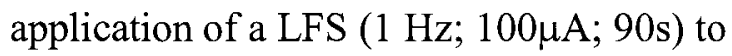

Treatment 1: the kindled amygdala with ADTs measured from the kindled discrete amygdala electrode.

Treatment 2: the spanning electrode with ADTs measured from the kindled discrete amygdala electrode.

Figure 17. Average ( \pm S.E.M.) percent change ADTs in the time following application of LFS $(1 \mathrm{~Hz} ; 300 \mu \mathrm{A} ; 300$ s) to the spanning electrode with ADTs measured from the kindled discrete

Figure 18. Average ( \pm S.E.M.) percent of seizure failure after the application of LFS in the following treatment conditions:

Treatment 1: LFS $(1 \mathrm{~Hz} ; 100 \mu \mathrm{A} ; 90 \mathrm{~s})$ applied to the kindled amygdala with ADTs measured from the kindled discrete amygdala electrode. Treatment 2: LFS $(1 \mathrm{~Hz} ; 100 \mu \mathrm{A} ; 90 \mathrm{~s})$ applied to the spanning electrode with ADTs measured from the kindled discrete amygdala electrode. Treatment 3: LFS $(1 \mathrm{~Hz} ; 150 \mu \mathrm{A} ; 90 \mathrm{~s})$ applied to the spanning electrode with ADTs measured from the kindled discrete amygdala electrode. Treatment 4: LFS $(1 \mathrm{~Hz} ; 300 \mu \mathrm{A} ; 90 \mathrm{~s})$ applied to the spanning electrode with ADTs measured from the kindled discrete amygdala electrode. 


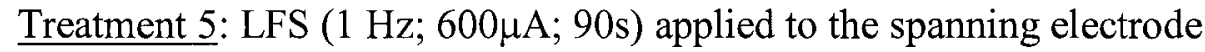
with ADTs measured from the kindled discrete amygdala electrode.

Treatment 6: LFS (1 Hz; 300 $\mu \mathrm{A} ; 300 \mathrm{~s})$ applied to the spanning electrode with ADTs measured from the kindled discrete amygdala electrode....78

Figure 19. Photomicrograph montage of histological staining of coronal sections after completion of LFS protocol showing:
A. Lack of pathology in the right pyriform cortex as shown with cresyl violet
B. Lack of pathology in the CA1 region of the hippocampus as shown with cresyl violet
C. Lack of pathology in the right pyriform cortex with as shown with fluorojade B stain
D. Small population of fluorojade B positive cells located around the electrode tip in the right basolateral amygdala nucleus.
E. Lack of pathology in the CA1 region of the hippocampus with Fluorojade B
F. Lack of pathology in the CA3 region of the hippocampus with Fluorojade B

Figure 20. Average ( \pm S.E.M.) percent changes in the ADTs with LFS $(1 \mathrm{~Hz} ; 300 \mu \mathrm{A}$; 300 s) applied to right amygdala electrode with ADT tested on the kindled

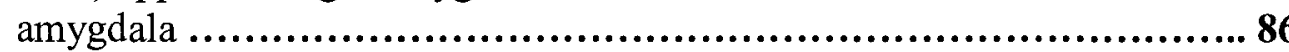

Figure 21. Average ( \pm S.E.M.) percent changes in ADT with LFS $(1 \mathrm{~Hz} ; 300 \mu \mathrm{A} ; 300 \mathrm{~s})$ applied to non-kindled spanning electrode with ADT tested on the kindled

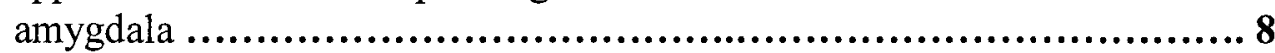




\section{LIST OF APPENDICES}

Appendix 1: Type and Location of Implanted Electrodes

109 


\section{LIST OF ABBREVIATIONS}

$\begin{array}{ll}\text { AD } & \text { After discharge } \\ \text { ADT } & \text { After discharge Threshold } \\ \text { AED } & \text { Antiepileptic drug } \\ \text { EBS } & \text { Electrical brain stimulation } \\ \text { EEG } & \text { Electroencephalogram } \\ \text { GABA } & \gamma \text { - Amino-butyric acid } \\ \text { ILAE } & \text { International League Against Epilepsy } \\ \text { LFS } & \text { Low Frequency Stimulation } \\ \text { LTD } & \text { Long Term Depression } \\ \text { NIH } & \text { National Institute of Health } \\ \text { SE } & \text { Status Epilepticus } \\ \text { TLE } & \text { Temporal Lobe Epilepsy }\end{array}$




\section{INTRODUCTION}

\section{EPILEPSY}

Epilepsy, with a worldwide prevalence of approximately $0.5-1 \%$, is one of the most common, acquired, chronic, neurological disorders (Pitkanen \& Sutula, 2002). The highest incidence of epilepsy is amongst juveniles and the elderly, although the disorder can manifest itself at any age. The term "epilepsy" envelops a plethora of seizure classes that vary widely in both their etiology and neuropathophysiology. Therefore, to try to ascribe a single defining label to this condition is neither practical nor theoretically sound. Instead, the "International League Against Epilepsy (ILAE)" has decided to use three elements, common to most seizure disorders, to describe epilepsy. These are: 1) a known history of at least one seizure; 2) permanent alteration in the brain and; 3) any associated neurobiological, cognitive, psychological, and social difficulties (Fisher el al., 2005).

The description of epilepsy by ILAE derives from the well-established knowledge that despite the many differences in the expression and the causal nature of epilepsy the fundamental aspects of the disease are common amongst its many different types. That is to say that the clinical manifestations of the various types of epilepsies, regardless of the cause, are due to the abnormal, recurrent bouts of electrical discharges from a group or groups of hyperactive neuronal populations (Treiman, 2001; Fritschy et al., 1999; Schwartzkrion \& Wyler, 1980). Furthermore, due to the recurrent nature of such events, epilepsy is considered a chronic disorder, with a transient and acute component to individual seizures, which clearly demarcate the beginning and end to the seizure event (Lothman et al., 1991).

Clearly then, any classification of epileptic seizure types presents a daunting task. To effectively differentiate the various forms of seizure disorders a classification system must 
include the differences in etiology, anatomy, physiology, progression and their unique clinical manifestations. Etiologically, the numerous seizure types have generally been differentiated into idiopathic and symptomatic. The majority of the former types of seizure disorders are benign, age-dependent and are believed to be due primarily to a genetic predisposition. Conversely, symptomatic epilepsy disorders stem from a wide array of causes that include both genetic and non-genetic neuropathological triggers. Common examples of the latter are brain abnormalities that may result from strokes, tumors, head trauma, congenital brain abnormality, scarring or "sclerosis" of brain tissue, cysts and infections (Morimoto et al., 2004).

Physiologically, the most commonly used categorizations of the seizure types are simple partial, complex partial and generalized. Simple and complex partial seizures are similar in terms of their aberrant electrical discharge region being localized in one hemisphere. They only differ in that the latter is usually characterized by the suspension of consciousness, which may or may not be accompanied by automatisms.

When hypersynchronous electrical brain activity recruits other brain regions in the generation of a seizure, it is called generalized. If such recruitment encompasses the entire brain, such a seizure is primary, as compared with a secondary generalized seizure, which usually develops over time as progressively greater areas of the brain become involved in the pathology. Loss of consciousness generally accompanies generalized seizures, which frequently conclude with a convulsive tonus (i.e. rigidity of the muscles due to their continuous contraction) and clonus (i.e. rhythmic jerking of body extremities). 


\section{Temporal Lobe Epilepsy}

Of all the reported cases of epilepsy, complex partial seizure disorders constitute approximately $25 \%$ of pediatric and $50 \%$ of adult cases, thus making it one of the most common epileptic disorders. In view of the fact that the majority of this seizure type originates within the temporal lobe, it is also referred to as temporal lobe epilepsy (TLE) (Loscher \& Schmidt, 1988).

The temporal lobes contain the limbic system and like most brain regions, it is functionally heterogeneous, and is responsible for the performance of many functions, including homeostatic control, emotions, memory and learning, as well as motor and sensory processing. Therefore, it is not surprising to learn that pathology resulting from temporal lobe epilepsy may give rise to behavioural deficits in consciousness, psychomotor and psychosensory functioning, emotional disturbances and impairments in learning and memory (Fendel, 1974).

Any event that jeopardizes the integrity of the limbic system may result in the development of the TLE. A number of pathophysiological triggers have been found to contribute to its genesis. These include, but are not limited to, major head traumas, cortical scarring, tumours, infections, gliomas, vascular malformations, strokes or even febrile seizures, which have the potential to act as triggers for the epileptic disorder either at the initial time of onset or sometime later in life.

Although a number of contributing factors to the development of TLE have been identified, the underlying causal mechanisms of complex partial seizure disorders are not known. It appears that it may be related to the breakdown of the balance between inhibitory and excitatory mechanisms within the epileptic focus. The matter becomes more complicated 
when multiple epileptogenic foci are involved. This poses a formidable challenge for physicians who rely upon pharmacological means of treating patients with TLE, as many cases (approximately 50\%) with this condition are medically intractable with traditional anticonvulsant and antiepileptic drugs, such as phenobarbital, carbamazepine and diphenylhantoin (Maynert \& Kusek, 1980; Hauser \& Kurland, 1975).

\section{Status Epilepticus}

A subgroup of the epilepsies that is of great concern, and is a medical and neurological emergency is status epilepticus (SE), which is associated with high rates of morbidity and mortality. Estimated incidence of SE in North America ranges from $10-41$ cases per 100,000 people per annum with mortality occurring in $20 \%$ of those cases (Manno, 2003). SE is defined as a series of seizures lasting for more than 30 minutes and represents a failure or malfunctioning of the intrinsic mechanisms involved in seizure termination (Manno, 2003; Coulter and DeLorenzo, 1999).

The occurrence of a protracted seizure, with or without a loss of consciousness, initiates an extensive spectrum of clinical symptoms that vary depending upon the etiology, anatomy and neuropathophysiology of the underlying seizure disorder (Scott et al., 1998). Hyperthermia, pulmonary edema, cardiac arrhythmias, and cardiovascular collapse can occur after generalized convulsive SE, thereby increasing the rate of mortality in these patients. Survivors of status events are not left unscathed, as long-term effects, such as epileptogenesis, encephalopathy and cognitive deficits, can be a consequence of the initial SE episode (Fountain, 2000). 
Presently, the predominant treatment of choice for SE is pharmacological. Some commonly used anti-epileptic agents are the benzodiazepines, phenytoin and phenobarbital (Manno, 2003). Even though these medications vary in terms of their unique physiological and pharmacokinetic properties, their anti-epileptic effects are induced by way of the cessation of a seizure activity and the prevention of recurrent seizures. Despite their relative success, none of the current therapeutic methods are able to provide the sufferer with complete treatment, let alone fully cure the disorder.

A more effective treatment would be one, which would directly affect the cause of the seizure initiation mechanism(s). Therefore, a better understanding of such a mechanism(s) would help enormously in the development of new and improved antiepileptic therapies. Although the crucial question as to what causes an epileptic disorder has yet to be answered, successful advances in this area of epilepsy research have been made. Much of the current knowledge about the various forms of epilepsy disorders, including SE, spring from successful development and subsequent applications of experimental animal models.

\section{EXPERIMENTAL MODELS OF EPILEPSY}

Animal models continue to be, for reasons both practical and ethical, the most valuable and widely used approach for the scientific investigation of many neurological diseases, including epilepsy. An ideal animal model of an epileptic disorder should show the following: spontaneous, recurrent seizures, seizures that mimic the human condition, age dependant onset, associated epileptiform activity on the electroencephalogram (EEG), pharmacokinetics of antiepileptic drugs (AEDs) similar to those used in the treatment of 
human epilepsies and effective plasma AED concentrations of a similar dosage found in the human condition (Sarkisian, 2001; Löscher \& Schmidt, 1988).

Some of the well-known and widely used animal models of epilepsy, which have at least partially satisfied the aforementioned criteria are: (I) genetic models such as the AE and the El mice (Löscher et. al., 1986; Seyfried \& Glaser 1985); (II) a number of chemically induced seizure models, which utilize a variety of chemical compounds, such as GABA antagonists (such as pentylenetetrazol, bicuculline, picrotoxin, penicillin)(Krogsgaard-Larsen et al., 1997; ), cholinomimetic drugs (e.g. pilocarpine) (Tu et al., 2005; Freitas et al., 2004) and excitatory amino acid receptor agonists (kainic acid) ( Ben-Ari \& Cossart, 2000; Sperk et al.,1985); (III) epileptogenic models using the topical application to cortical areas of alumina hydroxide; and (IV) electrically induced seizure models, such as kindling (Racine, 1972a, Racine, 1972b; Racine et al., 1972; Goddard et al., 1969).

These and many other animal models of epilepsy have been useful in the study of possible mechanism of epileptogenesis by the examination of how an epileptomimetic stimulus triggers an epileptic episode and through experimental manipulations carried out under controlled conditions of the progression of the seizure activity in the brain of an experimental animal (Coulter et al., 2002).

\section{The Kindling Model of Epilepsy}

For more than 100 years, physiologists have used electrical brain stimulation (EBS) to study brain functions. Often in studies utilizing this experimental procedure, scientists found that, while initial stimulations were without effect, repeated application of electrical current would sometimes produce convulsions in animals. This was largely dismissed as by- 
product and nuisance of such experiments and was purposely avoided. Graham Goddard first recognized the value of the phenomenon as an epilepsy model. Since Goddard's initial study (Goddard et al., 1996) in which he and his associates generated experimentally controlled epilepsy-like brain activity, the kindling model has been used extensively as a chronic animal model of TLE.

The kindling effect refers to the process whereby spaced and repeated (for approximately $2 \mathrm{sec}$ day) low intensity electrical stimulation of certain forebrain structures results in the progressive development of electroencephalographic and behavioural (i.e. convulsive) seizure activity (Goddard et al., 1969). There are a number of important experimental characteristics regarding the induction of the kindling effect, which sets it apart from all other models of epilepsy.

First, this model avoids the experimental confounds that are commonly seen in the chemically induced models of epilepsy, namely brain trauma and apoptosis. This is a crucial point, as it shows that the kindling effect is caused by a mechanism(s) inherent in the normal functioning of the nervous tissue and is not due to some secondary, pathophysiological process, such as edema, gliosis, alteration of the blood-brain barrier, acidosis and/or electrolytic effects of stimulation (Goddard et al., 1969).

Second, to effectively induce the kindling effect the frequency of stimulation must be set within a certain range, usually between $60-100 \mathrm{~Hz}$ (Goddard et al., 1969). This implies that the triggering frequency is an inherent property of the neurons being involved, which cannot be altered by repeated stimulation at other frequencies (Goddard et al., 1969). Third, the induction of the kindling effect only occurs with the generation of an evoked electrographic response called the afterdischarge (AD), so named because it continues 
independently after the stimulation offset. This means that the optimal electrical stimulus necessary to result in kindling must do more than merely activate the targeted neuronal network. It must tetanize the neural tissue to a hyperexcitable state sufficient to evoke $\mathrm{AD}$ (Goddard et al., 1969). This minimum amount of stimulation intensity is known as the AD threshold (ADT) (McNamara and Wada, 1997; Racine, 1972a; Goddard et al., 1969).

Fourth, with reapeated stimulation the characteristics of $\mathrm{AD}$ progressively change. Its threshold decreases and hence, currents of smaller intensities can be used to evoke AD (Racine, 1972b). Also, the waveform of the $\mathrm{AD}$ becomes more complex (i.e. of higher frequency) and larger in amplitude. The duration of $\mathrm{AD}$ becomes longer and more widely propagated. Most importantly, as kindling continues, $\mathrm{AD}$ propagates to other brain regions, resulting in secondary epileptogenesis (McNamara \& Wada, 1997). This is the rapid development of a second epileptic site in the brain, whose epileptogenic activity can be evoked by either electrical or pharmacological means.

Over time there occurs the progression of seizure activity from strictly electrographic in nature to secondarily generalized behavioural convulsions. Racine (1972b) devised a 5point kindling scale on the basis of the particular behavioural manifestations, which a kindled animal exhibited as it proceeded from the beginning to the end of the process. Stage 0: no behavioural change; Stage 1: behavioural arrest and facial clonus; Stage 2: Head nodding; Stage 3: controlateral forelimb clonus; Stage 4: rearing; Stage 5: rearing and falling.

The progression through the five aforementioned stages of kindling occurs in stepwise transitions from one state of neural organization to another (Burchfiel \& Applegate, 1989). Burchfiel and Applegate (1989) hypothesized that these transitions form the major steps in kindled seizure propagation and function as "neural gates," which underlie the 
functional reorganization of the kindled neural substrate. These gates divide the kindling process into three phases, with the initial one consisting of Racine's stages 1 and 2, the middle phase corresponding to stage 3 and the late phase, encompassing stages 4 and 5 .

This hypothesis implies that the propagation of an epileptic seizure may involve overcoming transynaptic thresholds. Indeed, the $\mathrm{AD}$ changes seen with repeated brain stimulation may be the electrographic correlatives of the neural changes that underlie this process. This is consistent with Goddard et al., (1969) concluded that the neural changes associated with the kindling process had an all-or-nothing character, such that they did not accelerate or were made more complete by higher stimulation intensities. It appears that the transynaptic spread of seizure depends on the cumulative effect of $\mathrm{AD}$, and only when the neural effects of $\mathrm{AD}$ reach a certain level, do the gates open to allow seizure to generalize to other brain sites, culminating in full-blown tonic-clonic convulsions.

Finally, the neural changes associated with the kindling effect are permanent (McIntyre et al., 1982; Goddard et al., 1969). That is, if left unstimulated a fully kindled brain remains highly prone to seizure activity and it takes only a few stimulations to generate convulsive behaviour in experimental animals (Goddard et al., 1969). For example, in a more recent study using primate subjects the persistence of the kindling effect has been observed to span at least two and a quarter years (Kulkarni \& George, 1994). It is this attribute of kindling that has made it a time-honoured model of human TLE.

\section{Clinical Validity of Kindling}

The kindling model has been the most widely used animal model of TLE since its inception some three decades ago. It has been shown to be robust for every animal species 
studied to date (frogs, reptiles, mice, rats, rabbits, dogs, cats, rhesus monkeys and baboons) (McNamara \& Wada, 1997). However, given the fact that any animal model is inherently limited by virtue of it being a biological simulation of the real phenomenon, a crucially important question is how relevant is the kindling model to the true human condition of secondarily generalized partial seizures?

The validity of the kindling model has been substantiated by the well-established physiological and behavioral similarities between kindling-induced epileptogenic effects and the human TLE. First, the behavioural patterns of stages 1 and 2 of kindled seizures as seen in experimental animals are comparable to those in humans with complex partial seizures of a temporal lobe origin. Such behavioral manifestations are arrest of ongoing activity, oral automatisms and reduced responsiveness to external stimuli (Kulkarni \& George, 1994; Sato et al., 1990).

Second, EEGs recorded from kindled amygdalae and hippocampi during a stage 5 experimental seizure are characterized by spike-and-wave and complex polyspike patterns, which are commonly seen in human complex seizures (Sato et al., 1990). Third, both kindled and human complex partial seizures respond similarly to anticonvulsant pharmacological agents (Sato et al., 1990; Albright \& Burnham, 1980). Fourth, the EEG interictal spiking, which is usually observed in the human condition and whose frequency is generally a good predictor of the severity of the TLE, also occurs in experimental animals kindled to human equivalent behavioral stages (Sato et al., 1990). Fifth, as in the human condition, the disposition to seizure activity is permanent (Pinel, 1981; McIntyre \& Goddard, 1973; Racine, 1972b; Goddard et. al, 1969). 
Finally, at least two pieces of evidence have shown that kindling is possible in humans. The first was a schizophrenic patient, who had received repeated electroconvulsive therapy and the second was based on the observations of a patient, suffering from severe chronic pain, whose condition was controlled by the electrical stimulation of his left thalamus. Both individuals developed motor seizures, which subsequently generalized (Sato et al., 1990).

\section{Kindling-based genetic predisposition}

Advances within the area of molecular neuroscience in recent years have led to an increase in the understanding of the role of genes in the epilepsies. Genes have been found to be causally implicated in rare idiopathic epilepsies, and genetic mutations have been identified in the epileptogenesis of some of the inherited symptomatic epilepsies (Kullman, 2005). Using the kindling model of epilepsy, coupled with selective breeding, Racine and associates developed seizure-prone (FAST) and seizure-resistant (SLOW) strains of rats to investigate possible genetic underpinnings of TLE (Racine et al., 1999). There are a number of electrophysiological, molecular and behavioral differences between these two strains of rats (Racine et al., 1999; McIntyre et al., 1999; Poulter et al., 1999; Mohapel and McIntyre, 1998).

For example, during the selective breeding process, kindling experiments showed that FAST rats achieved seizures with daily amygdala kindling in approximately a week, while SLOW rats required three weeks or more to reach tonic-clonic convulsions (stage-5 seizures) (Racine et al., 1999). The separation between the two strains in their susceptibility to amygdala kindling became significant as early as the $2^{\text {nd }}$ generation of selective breeding and 
there was no overlap between the kindling rate distributions between the animals as early as the $6^{\text {th }}$ generation. Selection for amygdala kindling was stopped at the $11^{\text {th }}$ generation, and random breeding within each strain has occurred since that time.

McIntyre et al., (1999) also found that when amygdala kindling rates between the FAST and SLOW animals were compared, the former required considerably fewer stimulation trials to reach stage 5 seizure. This differential responsivity of the two strains to kindling did not stem from the mechanisms that controlled amygdaloid ADTs of FAST versus SLOW rats, as this measure was similar between the two strains. Rather it was the mechanisms that regulated the propagation of $\mathrm{AD}$ from the focus to other brain structures that appeared to have been responsible for the strain differences (Racine et al., 1999; McIntyre et al., (1999). Interestingly, however, post-stimulated FAST rats had considerably lower ADTs compared with their SLOW counterparts, suggesting that after kindling the seizure-prone FAST rat might be in a chronically sensitized state.

In relation to seizure latencies (i.e. the time between stimulation and appearance of first convulsions on any given trial), FAST rats exhibited significantly higher values than their SLOW counterparts. At first glance, this may seem counterintuitive, but if longer AD durations (i.e., the duration of the hypersynchronized neural activity from the time of stimulation to the end of stage-5 seizure) of FAST rats, relative to SLOW rats, are taken into account, then longer latencies for this strain may be explained in terms of a protracted period of time, which is necessary for more extensive recruitment of neural networks to trigger tonic-clonic convulsions (McIntyre et al., 1999). There is apparently a trade-off between the number of stimulations required to trigger convulsions and their behavioural manifestations in FAST rats, such that fewer trials are required to produce Stage-5 seizures, but because 
each stimulation causes a greater recruitment of networks, the onset of such seizures is later rather than earlier in FAST, compared with SLOW rats.

Attempts have been made to explain the aforementioned results in terms of the intrinsic inhibitory receptor mechanisms of SLOW rats being overactive while those of FAST rats being hypoactive. Molecular evidence appears to support this hypothesis. $\gamma-$ Amino-butyric acid (GABA) is the major inhibitory neurotransmitter whose functions are mediated via GABAergic protein receptors, made of several sub-units, namely $\alpha, \beta, \gamma, \delta, \rho$, whose specific combinations determine their functions (Longstaff, 2000). Poulter et al., (1999) found that particular subsets of the $\alpha$ subunits were differentially expressed in the FAST versus the SLOW rats.

In particular, the $\alpha_{2}, \alpha_{3}, \alpha_{5}$, which are normally expressed during embryonic development, are in greater abundance in several limbic brain regions (e.g., amygdala but not the hippocampus) of FAST rats compared with SLOW rats and controls. SLOW rats on the other hand, had over-expression of the adult subunit, $\alpha_{1}$, compared to both FAST and control rats. The authors concluded that the major predisposition of the FAST rats to kindling might be due to its failure to complete the normal development from the embryonic to adult phenotype of its $\mathrm{GABA}_{\mathrm{A}}$ receptors (Poulter et al., (1999).

Behaviourally, there are also differences between non-kindled SLOW and FAST rats, which likely stem from the differences in their brain, particularly limbic, physiology. For example, Mohapel and McIntyre (1998) in a number of experiments showed that FAST and SLOW rats differed significantly in a number of behavioural paradigms, including exploration of novel and familiar environments, as well as in reactivity to footshock and fearbased learning. 
The SLOW rats exhibited lower ratio of open (anxiogenic) - to closed arm entries in the elevated plus-maze paradigm, than the FAST animals. Also, they showed reduced activity over days in the open field paradigm and even greater behavioural suppression (freezing) if previously shocked than did their FAST counterparts. The results of these experiments were interpreted that the SLOW strain was more fearful than the FAST.

\section{ELECTRICAL STIMULATION IN THE EPILEPSIES}

Pharmacological epilepsy treatment approaches have only been partially successful. In some cases severe side effects of anticonvulsive and antiepileptic medications makes them the least likely choice of therapy. There are also certain forms of seizure disorders that are resistant to the effects of such medications. Thus, there is a great need to develop nonpharmacological treatment alternatives for these types of epilepsies. A consensus completed by the National Institute of Health $(\mathrm{NIH})$ estimated that intractable seizures occur in roughly $20 \%$ of epileptic patients (Bendabis and Tatum, 2001).

Presently, the current treatment for most intractable seizures is resection of the region containing the epileptogenic focus, as this generally serves to reduce the frequency of seizure occurrence. However, in some cases, resection may not be possible as the epileptogenic focus may be in close proximity to functionally important brain regions and therefore would leave patients with severe cognitive, sensory and/or motor deficits (Durand and Bikson, 2001; Sun et al., 1998). Hence, other less invasive approaches have been researched and experimented with, one of which has been electrical brain stimulation (EBS).

EBS in humans has been in use for over 50 years. Traditionally it has been applied to the nervous system for the purpose of studying its basic functioning both centrally as well as 
in the periphery. (Durand and Bikson, 2001). More recently, minute electrical currents directed to central regions deep within the brain have been found to be of therapeutic value in treating a wide range of neurological and mental illnesses (Nilsson et al., 2005; Kerrigan et al., 2004; ) Over the last few decades the use of electrical stimulation to inhibit seizure expression has generated a lot of interest, especially as an alternative treatment for epilepsy.

Some experimental protocols have demonstrated suppression or interference with the abnormal electrographic discharges that characterize epilepsy. Reports have been made that when repeated electrical stimulations were administered to specific brain regions, this interference can be seen as a permanent change in seizure ADTs without any associated memory deficits (Vonk et al., 2002; Velasco et al., 2001; Velasco et al., 2000).

\section{Low Frequency Stimulation (LFS)}

Through the use of the kindling model of epilepsy it is has been well established that repeated application of brief high-frequency stimulation, $60 \mathrm{~Hz}$, to certain brain regions (e.g. the amygdala), results in the generation of fully expressed electrographic and behavioral seizures. In contrast, however, recent studies have shown that the application of lowfrequency stimulation (LFS), following kindling of the amygdala, disrupts and/or suppresses the generation of stage- 5 seizures and also increases the ADT (Goodman et al., 2005; Velíšek et al., 2002; Weiss, et al., 1998, Gaito \& Gaito, 1981). Gaito, whose findings were initially overlooked due to shortcomings in the experimental design and the poor presentation of the data, undertook initial studies in this area. Despite, the many experimental drawbacks, Gaito's studies demonstrated that LFS $(1 \mathrm{~Hz}$ or $3 \mathrm{~Hz})$, of varying durations, interfered with the kindling effect, mainly by means of an as yet unknown mechanism that causes an increase in the $\mathrm{ADT}$ necessary to trigger seizure activity in the targeted brain regions (Gaito, 1984; 
Gaito, 1981; Gaito \& Gaito, 1981; Gaito, 1980a; Gaito, 1980b; Gaito et al., 1980). In addition, it was also found that LFS effects lasted for several weeks, thus demonstrating a certain measure of permanence. Nevertheless, due to flaws in the experimental design of the study, it was not clear if these findings were confounded by possible brain damage, as no histological analyses were performed (Gaito, 1984).

Weiss et al, 1998 termed the suppression of the excitability in the kindled focus "the quenching effect." These researchers believed it to result from a complete blockage of epileptogenesis and progression of the associated $\mathrm{AD}$. If the quenching stimulus was applied on a daily basis, without concurrent kindling, resumption of the kindling stimulation did not result in seizures. The authors interpreted the findings as being due to the increase in the associated ADT, which resulted in a long-term blockage of the epileptogenic activity of the neural circuitry (Weiss et al., 1995).

However, once again the results were confounded by errors in the experimental design, as Weiss and associates used high intensity, direct current, square waves to induce LFS effects, which are known to produce lesions. Subsequent histological examination of the stimulated brain sites did, indeed, show extensive pathology as a result of the square wave direct current (Weiss et al, 1998). Thus the research community lost all interest in their LFS protocol.

Due to the contradictory results of the previous studies, a number of questions naturally arise. What LFS parameters are necessary for potential suppressive effects of seizure activity? How specific is the suppression effect of epileptogenesis? What is the time frame of the suppressing effect of LFS and does it generalize to other brain regions, both proximal and distal? These are questions that must be settled before LFS can be adapted for 
use on humans. Nonetheless, low frequency biphasic electrical stimulation presents a potentially promising novel therapeutic tool in the treatment of intractable forms of epilepsy.

\section{Purpose of this study:}

In general the study was revisited the question of whether or not LFS would produce true antiepileptic effects. More specifically the aims were twofold: 1) to examine the effects of the LFS on ADTs in terms of its spatial characteristics; and 2) to address the possible genetic underpinnings of the LFS using genetically predisposed (FAST) and resistant (SLOW) strains of rats. 


\section{MATERIALS AND METHODS}

\section{GENERAL}

Treatment of animals was done in accordance with the Animals for Research Act and the Guidelines of the Canadian Council on Animal Care. All experimental research protocols were approved by the Carleton University Animal Care Committee.

\section{Animals}

FAST and SLOW male rats were born and raised in the Carleton University Life Sciences and Research Building. Test subjects weighed between $250-450 \mathrm{~g}$ pre-surgery and were individually housed in cages with water and food provided ad libitum. The temperature $\left(21^{\circ} \mathrm{C}\right)$ and humidity $(45-50 \%)$ were maintained at a constant level with a 12 hour lightdark cycle.

\section{EXPERIMENT 1 - KINDLING AND STABILITY OF ADTs}

\section{Animals}

Originally, 6 FAST and 7 SLOW male rats were assigned to this study and underwent stereotaxic surgery for electrode implantation. Animals that lost their headplug assembly $(\mathrm{N}=1, \mathrm{FAST})$ post-surgery were removed from the study.

\section{Surgery}

Rats were initially sedated with Halothane and then anesthetized with sodium pentobarbital (Somnotol; 60mg/kg) injected interperitoneally (i.p.) and supplemented with Halothane if needed. Ground electrodes, consisting of male Amphenol contacts attached via 
wires to steel jeweler's screws, were inserted into the skull at $10.0 \mathrm{~mm}$ anterior to bregma (see Appendix 1 for full description of coordinates) and 6 jewelers screws were secured to the skull prior to electrode implantation to lend anchorage to the headplug assembly (Molino and McIntyre, 1972).

Twisted, bipolar stimulating/recording electrodes made of Nickel-Chromium were stereotaxically implanted in both right and left amygdalae using a Kreig stereotaxic apparatus. These twisted electrodes, called the discrete electrodes, were of a traditional configuration in which the paired twisted wires were separated by the thickness of the Diamel insulation. Another electrode, which was a combined unit consisting of two twisted electrodes arranged to create one spanning electrode (Figure 1), was implanted in the amygdala-piriform complex such that it spanned the discrete electrodes in each hemisphere (Figure 2).

Bilateral implantations were done as a preventative measure on the off-chance that the electrodes in one hemisphere were unusable due to inaccurate electrode implantation or pain responses being elicited on stimulation. The stereotaxic co-ordinates (Pellegrino et al., 1979) used for the electrode implantations were listed in Appendix 1. Dental acrylic was used to cover the surgical area, and electrodes were then carefully inserted into a 9 pin "McIntyre headplug" assembly. Prior to the initiation of kindling, animals were allowed a post-operative recovery time of $7-14$ days, but were handled on a daily basis beginning two days post-surgery. 
Figure 1 - Configuration of the "traditional" twisted, discrete electrode and spanning electrode. 


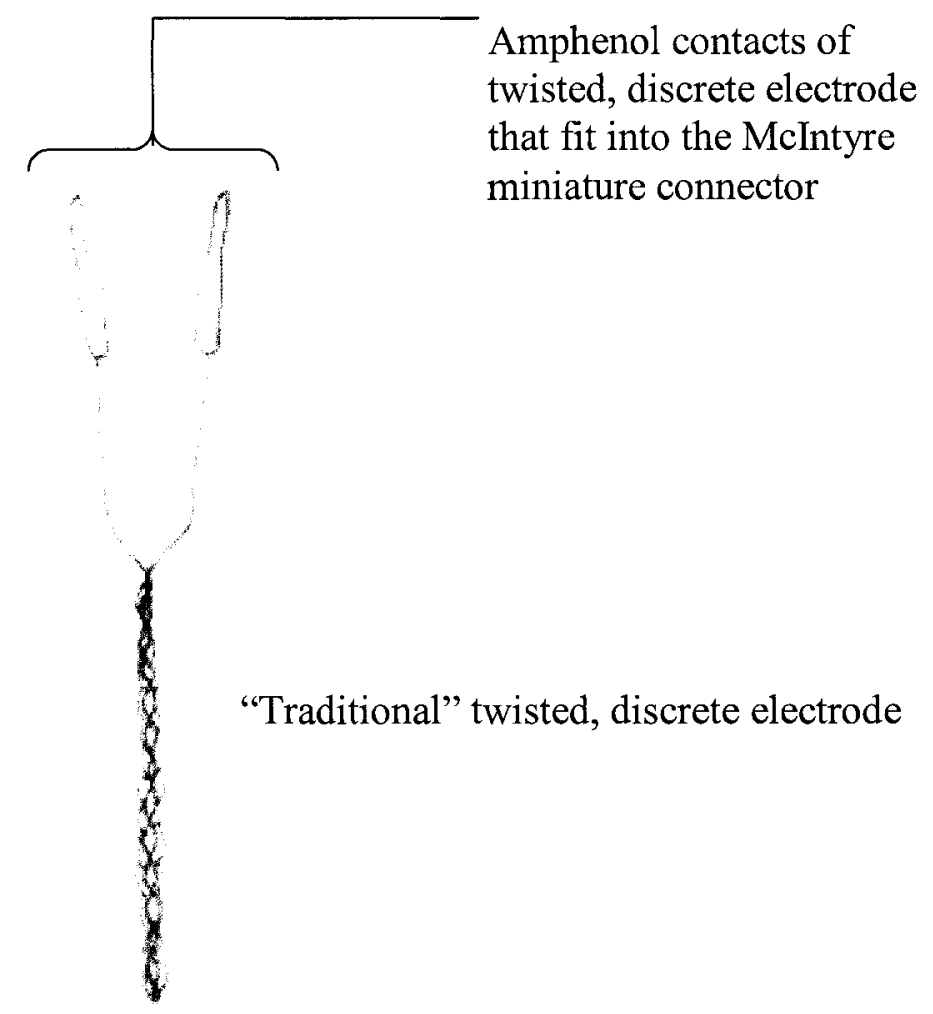

These two Amphenol pins of the corresponding electrodes were inserted into the McIntyre miniature connector to correspond to one stimulating and recording electrode for the

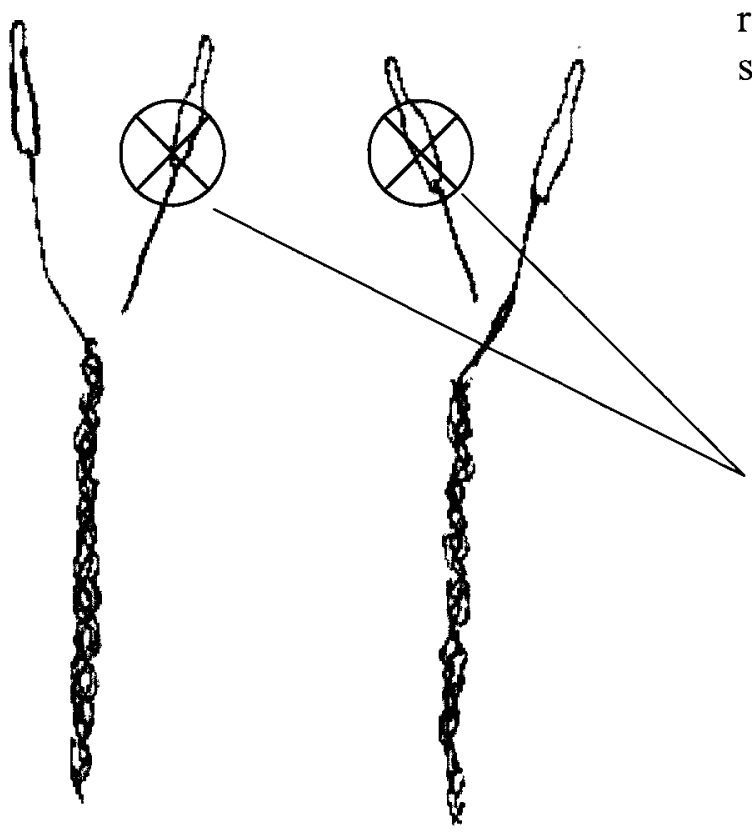

Both parts

removed to

create one

spanning

electrode 
Figure 2 - Schematic representation, (Paxinos and Watson, 2005) of the electrode placements showing targeted regions. The red circle corresponds to the ground electrode. The blue circles correspond to the amygdala electrode and the green circles correspond to the spanning electrodes. The upper coronal section, at $2.0 \mathrm{~mm}$ anterior to bregma, represents the region where the first tip for the spanning electrode was inserted into the piriform area. The middle coronal section, at $0.0 \mathrm{~mm}$ bregma, represents the electrode tip for the twisted, discrete electrode placed into the amygdaloid complex. The bottom coronal section, at $2.0 \mathrm{~mm}$ posterior to bregma, represents where the second electrode tip for the spanning electrode was inserted within the amygdala/piriform cortex. The figure to the left shows the insertion points of the electrodes in relation to the bregma on the rat skull. 


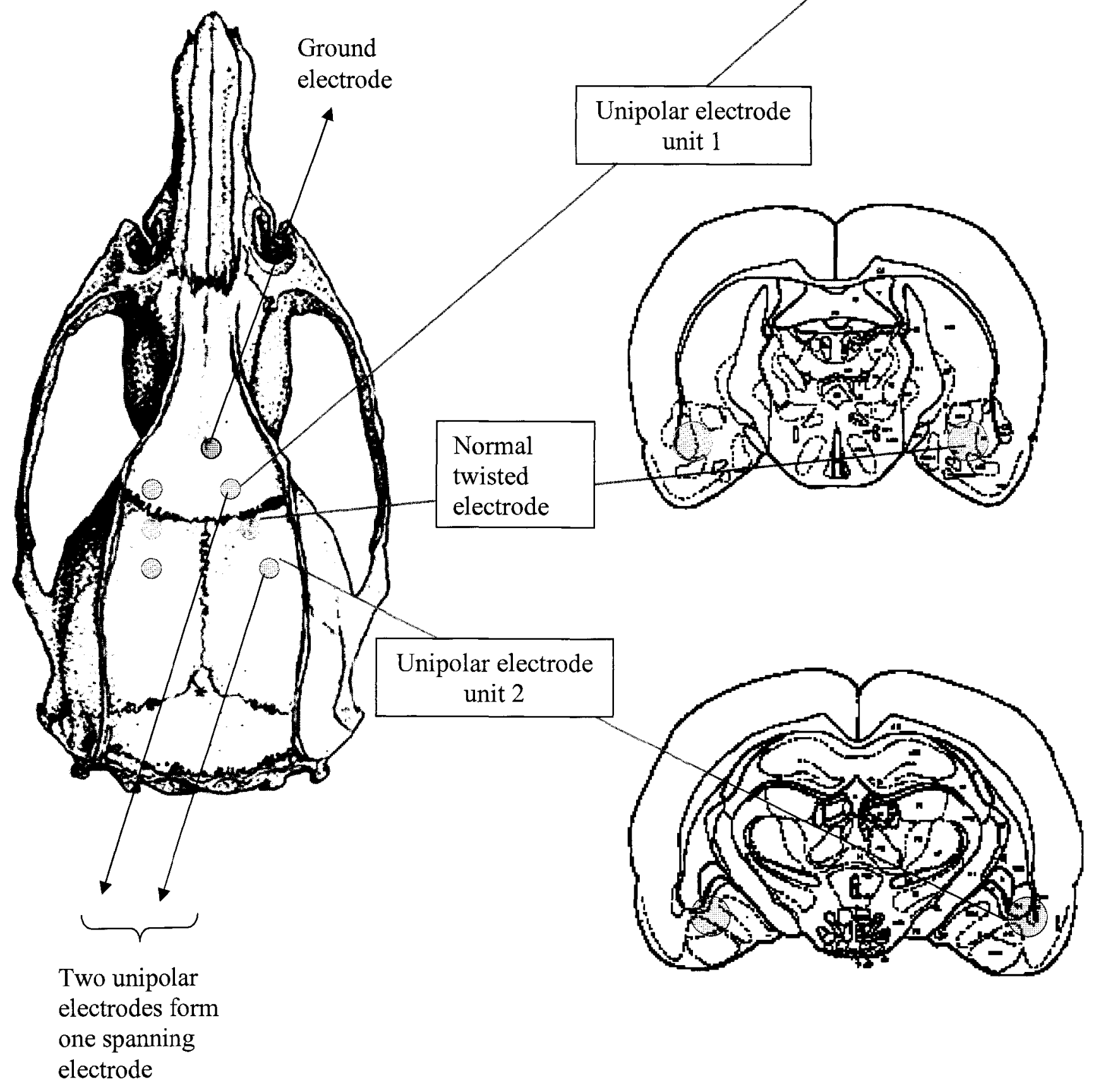




\section{Kindling and Afterdischarge Thresholds}

The level of excitability in a local neuronal circuitry can be measured by the ADTs, which are the minimum electrical stimulus intensity necessary to elicit ADs that outlast the stimulus itself by 2 or more seconds (McIntyre et al., 1999). Before kindling was initiated pre-kindling ADTs were measured. ADTs were determined by providing an ascending series of $60 \mathrm{~Hz}$ sine wave stimulations $(15,25,35,50,75,100,150,200,250,300,400$ and $500 \mu \mathrm{A}$ peak to peak) to the targeted brain regions in the rats for 2 seconds until ADs were elicited.

Each stimulation was separated by an interval of 1 minute. An interval of 5 minutes was allowed between ADT measurements from the twisted, discrete left amygdala electrodes and that of spanning left electrodes. Only the left electrodes were used unless an ADT was not found on the left side of the brain or the animal exhibited a pain response on those electrodes. In that case the right hemisphere electrodes were used.

AD latencies and durations were recorded on a Grass Model 7 Polygraph with normal kindling on the left discrete amygdala electrode commencing 24 hours after the ADT determinations. The kindling process involved stimulation of the left amygdala once daily at the ADT until 6 stage 5 generalized convulsive seizures were recorded. After the acquisition of these 6 stage 5 seizures, ADTs were again determined for the left amygdala over the course of several days to guarantee stability. Once the animals were kindled a convulsive seizure could be reliably elicited by administering a stimulus at the ADT intensity on the kindled left amygdala. 


\section{Low Frequency Stimulation Manipulations}

1) LFS administered to kindled left amygdala with ADT tested on kindled left amygdala:

Once stability of the ADTs of the kindled rats was ascertained, LFS manipulations were carried out. Each of the 12 male rats (one rat from the original $\mathrm{N}=13$ was removed from the study due to loss of the headplug assembly) was administered LFS of $1 \mathrm{~Hz}$ at $100 \mu \mathrm{A}$ intensity to the kindled left amygdala electrode for 30 seconds followed by a 1 min rest interval before re-determining the ADTs of the left amygdala. This manipulation was done to assess the near 'immediate' effect ( $60 \mathrm{sec}$ later) of LFS on the kindled amygdala. Of course, reaching the ADT would not only trigger a focal discharge, but also a convulsive seizure.

Measurements of the latency and duration of the motor seizure were noted on the EEG polygraph. The ADTs were then re-tested on the kindled left amygdala 24 hours later and then once daily until stable ADTs were re-acquired. When the ADTs of the kindled amygdala on re-testing no longer varied on a daily basis for at least three consecutive days, another LFS manipulation was administered. Using the same intensity $(100 \mu \mathrm{A})$ and frequency $(1 \mathrm{~Hz})$ the LFS stimulation was then administered to the kindled left amygdala for 90 seconds. This was then followed by a 1 minute interval after which the ADTs were found for that day and successive days until stable ADTs were again determined.

2) LFS administered to non-kindled left spanning area with ADT tested on kindled left amygdala:

Subsequently, application of LFS $(1 \mathrm{~Hz} ; 100 \mu \mathrm{A})$ was directed to the non-kindled left spanning area, in the same animals, with the resultant ADTs tested on the kindled left 
amygdala, using the discrete electrode, for the 30 and 90 second manipulations carried out in the same manner as previously described.

3) LFS administered to kindled left spanning area with ADT tested on kindled left spanning area:

Following the completion of the above LFS treatments via the discrete electrode, the animals were then kindled via the previously non-kindled spanning electrode, using the same kindling protocol, as was used with the left amygdala discrete electrode. Once stable values for the ADTs were acquired for three consecutive days LFS manipulations were performed. The first manipulation involved the application of the LFS $(1 \mathrm{~Hz} ; 100 \mu \mathrm{A})$ of 30 seconds to the kindled left spanning electrode, followed by a 1 minute interval before determining the values of the ADTs. Following this, the ADTs of the now kindled left spanning area was found until the value had stabilized and showed no variability on at least three successive days. The LFS $(1 \mathrm{~Hz} ; 100 \mu \mathrm{A})$ was then applied to the kindled left spanning electrode for 90 seconds and again ADTs were found 1 minute after the LFS and then on subsequent days until the ADTs were no longer fluctuating.

4) LFS administered to kindled left spanning area with ADT tested on previously kindled left amygdala:

The experimental procedure was the same in all respects to (3) above except that the recording of the ADTs were taken from the discrete electrode in the kindled amygdala. 


\section{Histology}

At the completion of the experiment the animals were perfused intracardially with $0.9 \%$ saline followed by $4 \%$ paraformaldehyde. Prior to sectioning, the brains were submerged in a $30 \%$ sucrose solution for 5 days. The brains were then frozen and sectioned coronally at $40 \mu \mathrm{A}$ on a microtome. The sections were mounted, dried and then stained with cresyl violet to confirm electrode placements and to assess for any possible pathology. The cresyl violet stain procedure involved passing slides of the mounted sections through a series of steps in the following order: I. dehydration by submersion in a series of ethanol baths of increasing concentrations ( $70 \%, 95 \% 100 \%)$ for 2 minutes each; II. a 2 minute immersion in clearene to dissolve lipids; III. a 2 minute immersion in ethanol baths of descending concentrations (100\%, 95\%, 70\%); IV. a 15 second immersion in 1\% cresyl violet stain; V. rinsed twice in distilled water to remove excess stain; VI. dipped in differentiator (acetic acid solution) until appropriate level of staining was achieved; VII. re-rinsed twice in distilled water; VII. dehydrated with ethanol baths $(70 \%, 95 \%, 100 \%(2 \mathrm{x}))$; VIII. 2 changes of clearene for 2 minutes; VIV. submerged for 3 minutes in clearene and then each slide was coverslipped with Fisher Permount Mounting Medium, as they were removed from clearene.

\section{Statistical Analysis}

Data were analyzed using Windows StatView 5.0 (SAS Institute Inc, Cary, NC, USA) and graphical representations of data were done using SigmaPlot 2000 for Windows 6.0 (Systat Software, Inc. Point Richmond, CA, USA). Kindling rates were evaluated by using ANOVA analysis and changes in ADTs over time were evaluated using repeated measures ANOVA with a p-value $<0.05$ considered to be statistically significant in both cases. For 
lack of a better control the stability of the ADTs post-kindling was used in comparison studies. Graphs, measuring changes in ADT, were assigned a baseline value. This value represented the original pre-testing value (i.e. raw scores) from which all changes in ADT were calculated. Hence, analysis of the changes in ADT involved the conversion of the resulting change in the variable as a percent of the baseline using the following equations:

i)

$$
\left[\frac{\text { Raw Score ADT (Baseline) }}{\text { Mean Raw Score ADT (Baseline) }}\right] * 100=\text { Baseline }(\%)
$$

ii)

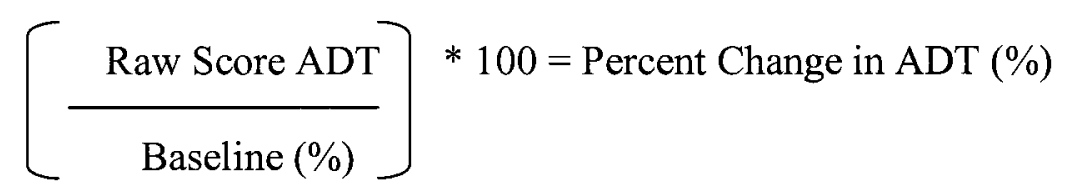

It should be noted that the stable ADT inter-experimental values obtained during consecutive administrations of the LFS were used as baseline measures for subsequent experimental conditions.

\section{RESULTS}

\section{Kindling Rates and Afterdischarge Thresholds}

Analysis of left amygdala kindling rates through the discrete electrode showed a significant main effect of strain $\left(\mathrm{F}_{1,10}=22.2, \mathrm{p}<0.0008\right)$ with SLOW rats requiring a considerably greater number of stimulations than the FAST rats. However, there was no strain difference in the kindling rates via the left spanning electrode after the kindling and LFS manipulations were carried out using the discrete electrode. Interestingly, there was a 
significant difference $\left(\mathrm{F}_{1,8}=15.1, \mathrm{p}<0.0046\right)$ in the kindling rates between the two types of electrodes, namely that the region stimulated by the left spanning electrode required fewer stimulations to trigger a stage 5 convulsive seizure than the previously kindled amygdaloid region (Figure 3). 
Figure 3 - Average ( \pm S.E.M.) number of stimulations required to elicit the first stage 5 seizure (i.e. kindling rate) for the FAST and SLOW strains as a function of the kindling site.

'*' = significantly different kindling rates of the SLOW vs. the FAST rats on the kindled left amygdala, recorded from the discrete electrode $\mathrm{p}<0.0008$.

' + ' = significantly different kindling rates of the discrete vs. the spanning electrodes of SLOW and FAST rats $\mathrm{p}<0.05$. 


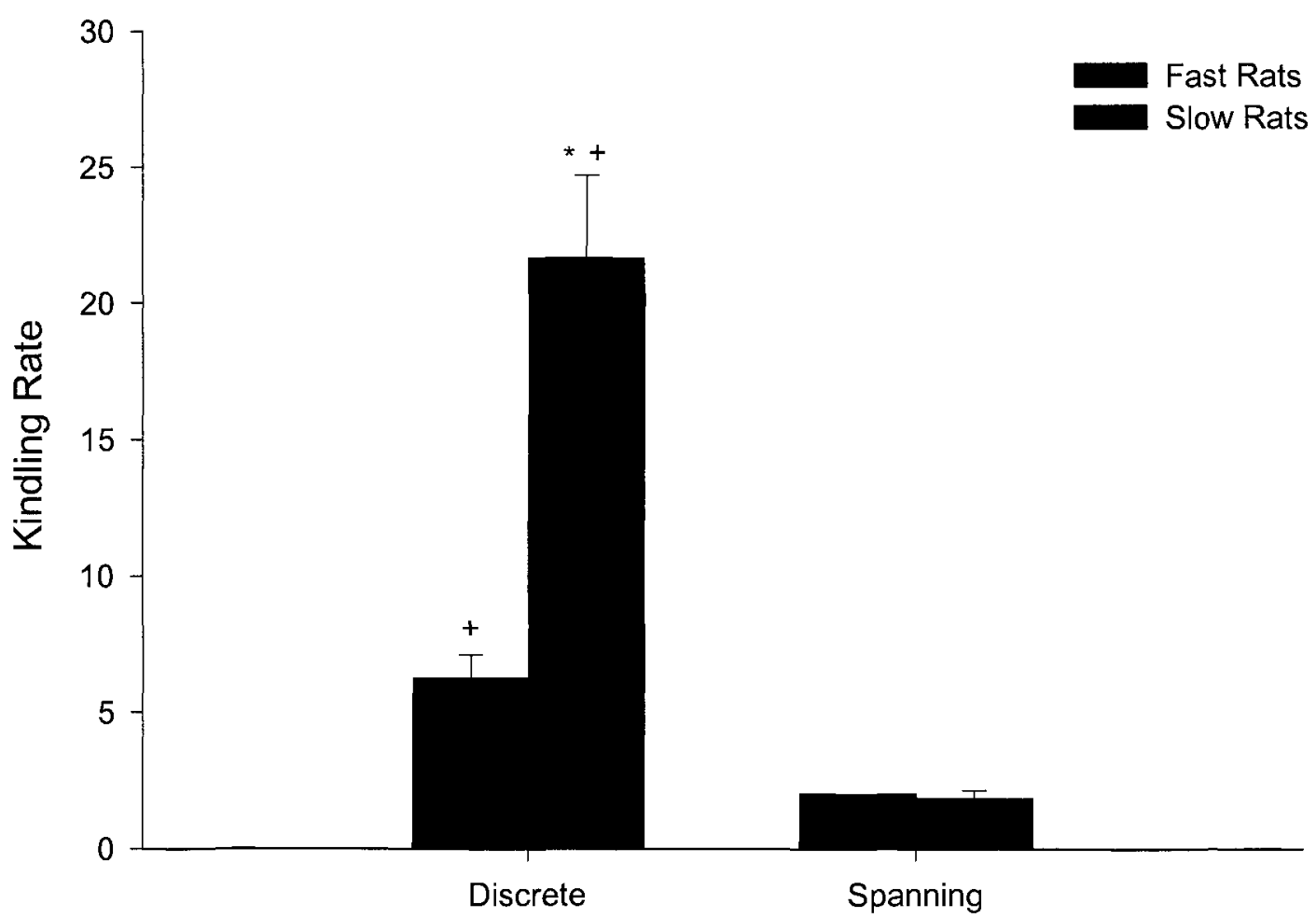


Analysis of the pre-kindled and post-kindled ADT conditions showed no significant strain effect, regardless of the area that was kindled. There was, however, a significant effect before and after kindling in the ADT measured from the discrete electrode $\left(\mathrm{F}_{1,8}=11.4, \mathrm{p}<\right.$ 0.01) (Figure 4). No such differences in pre- and post- kindling ADT were observed from the spanning electrode. Although there was a significant ADT drop $(\mathrm{p}<0.0016)$ from the prekindled to Day 1, post-kindled condition in both strains, no significant changes in the ADTs over the subsequent trials (i.e. Day 1 to Final Day 6) were observed (Figure 5). 
Figure 4 - Average ( \pm S.E.M.) changes in pre and post- kindling ADT of the left amygdala recorded from the discrete and spanning electrodes.

' $*$ ' = significant difference between the pre - vs. the post-kindled ADTs for the left discrete amygdala electrode, $\mathrm{p}<0.01$. 


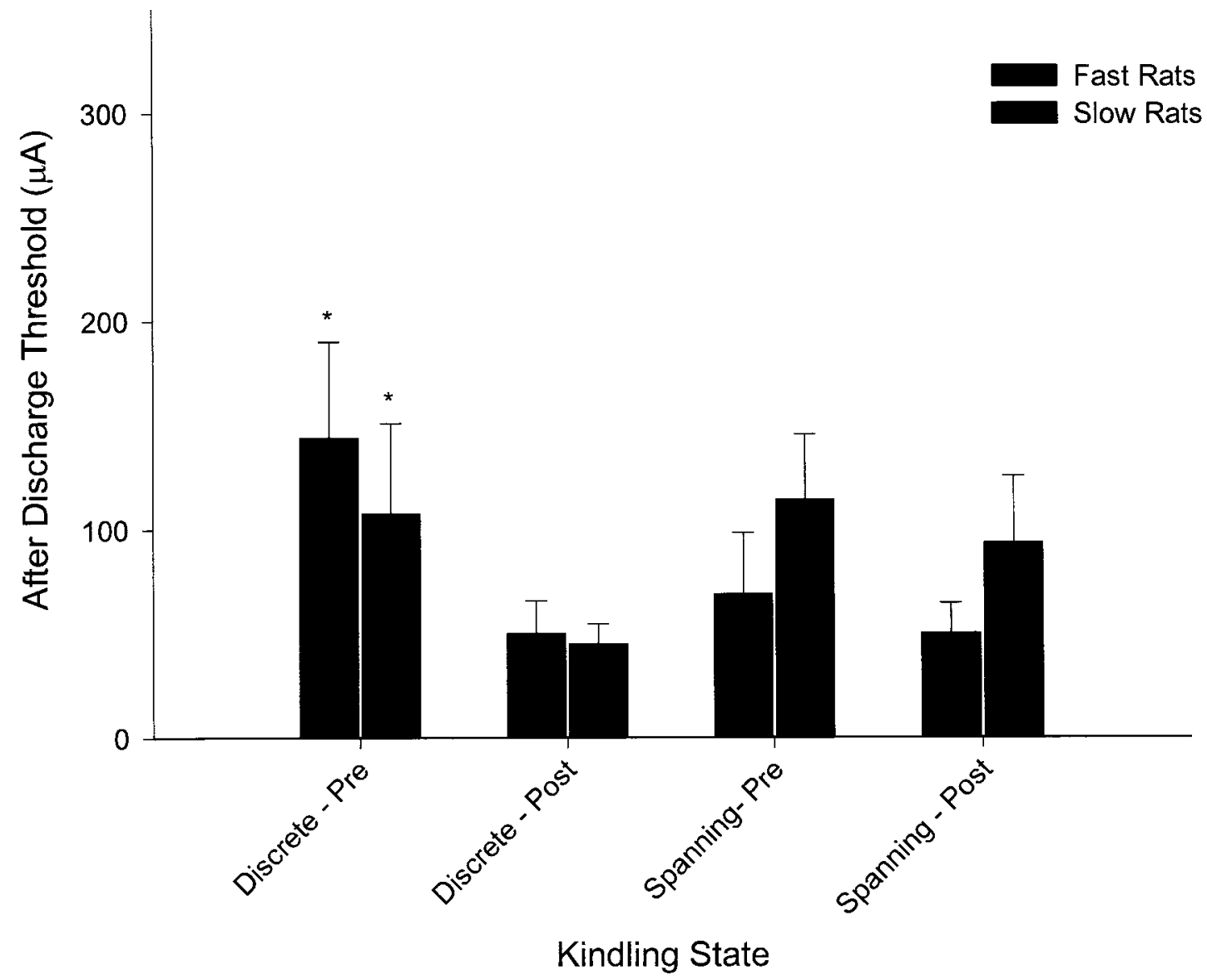


Figure 5 - Average ( \pm S.E.M.) percent change in the ADTs in the days following the establishment of the kindled state.

' $*$ ' = significantly different from the post kindled ADTs for the left discrete amygdala electrode for FAST and SLOW rats , $\mathrm{p}<0.0001$. 


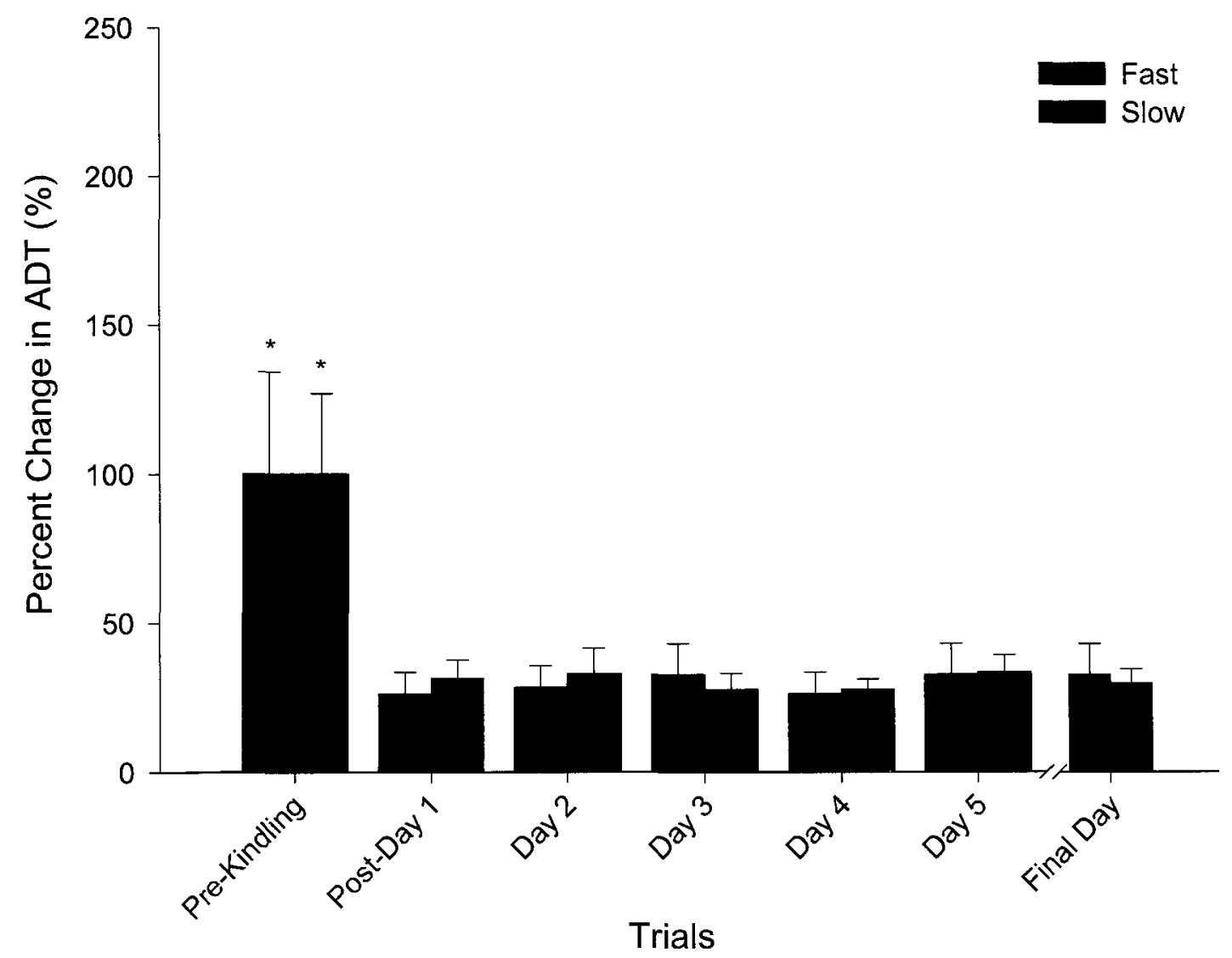




\section{Low Frequency Stimulation Manipulations}

1) LFS administered to kindled left amygdala with ADT tested on kindled left amygdala:

For the $30 \mathrm{sec}$ LFS there was no significant main effect or interaction effect of strain. The analysis of ADT for the days following the application of the LFS in the two strains showed a significant effect over trials $\left(\mathrm{F}_{1,7}=2.6, \mathrm{p}<0.02\right)$. The thresholds showed an immediate increase within 1 minute of the LFS stimulation. Follow-up comparisons, using the Newman-Keuls post-hoc test at $\alpha=0.05$ indicated a significant difference of the ADTs from baseline values on Day 1 and Day 2, p $<0.019$ (Figure 6a). The highest percent change from baseline in ADT over the days subsequent to the LFS occurred on Day $2(174.4 \pm 29.7$ \%) when collapsed over strain. By the final day of testing ADTs for the FAST rats were closer to the baseline values than the SLOW rats, which remained at a slightly elevated threshold $(123.8 \pm 22.3 \%)$ from their baseline value.

After the $90 \mathrm{sec}$ LFS stimulation administered to the kindled left amygdala via the stimulating discrete electrode, there were no significant interaction effects or main effects of strain in ADTs, as measured from the recording discrete electrode. However, there was a significant trial effect $\left(\mathrm{F}_{1,7}=3.39, \mathrm{p}<0.007\right.$, Figure $\left.6 \mathrm{~b}\right)$ within 1 minute post-LFS during which ADTs rose by $138.6 \pm 13.3 \%$ from baseline, followed by subsequent increases over the next 4 days of testing. The greatest percent change in ADTs occurred on Day 1 for FAST $(154.5 \pm 18.2)$ and SLOW $(172.6 \pm 48.1)$ rats. Thresholds returned to below baseline values for the FAST $(89.1 \pm 13.3 \%)$ on the last day of ADT testing whereas the SLOW group had a final ADT that remained above the baseline value $(138.7 \pm 35.5 \%)$. 
Figure 6 -a) Average ( \pm S.E.M.) percent change in the ADTs in the time following application of LFS $(1 \mathrm{~Hz} ; 100 \mu \mathrm{A} ; 30 \mathrm{~s})$ to the kindled discrete amygdala with ADTs measured from the kindled amygdala electrode.

'*' = significantly different from baseline ADTs for the left discrete amygdala electrode for the FAST and SLOW rats , $\mathrm{p}<0.0190$.

b) Average ( \pm S.E.M.) percent change in the ADTs in the time following application of LFS ( $1 \mathrm{~Hz} ; 100 \mu \mathrm{A} ; 90 \mathrm{~s})$ to the kindled discrete amygdala with ADTs measured from the kindled amygdala electrode.

'*' = significantly different from baseline ADTs for the left discrete amygdala electrode for the FAST rats, $\mathrm{p}<0.0075$. 

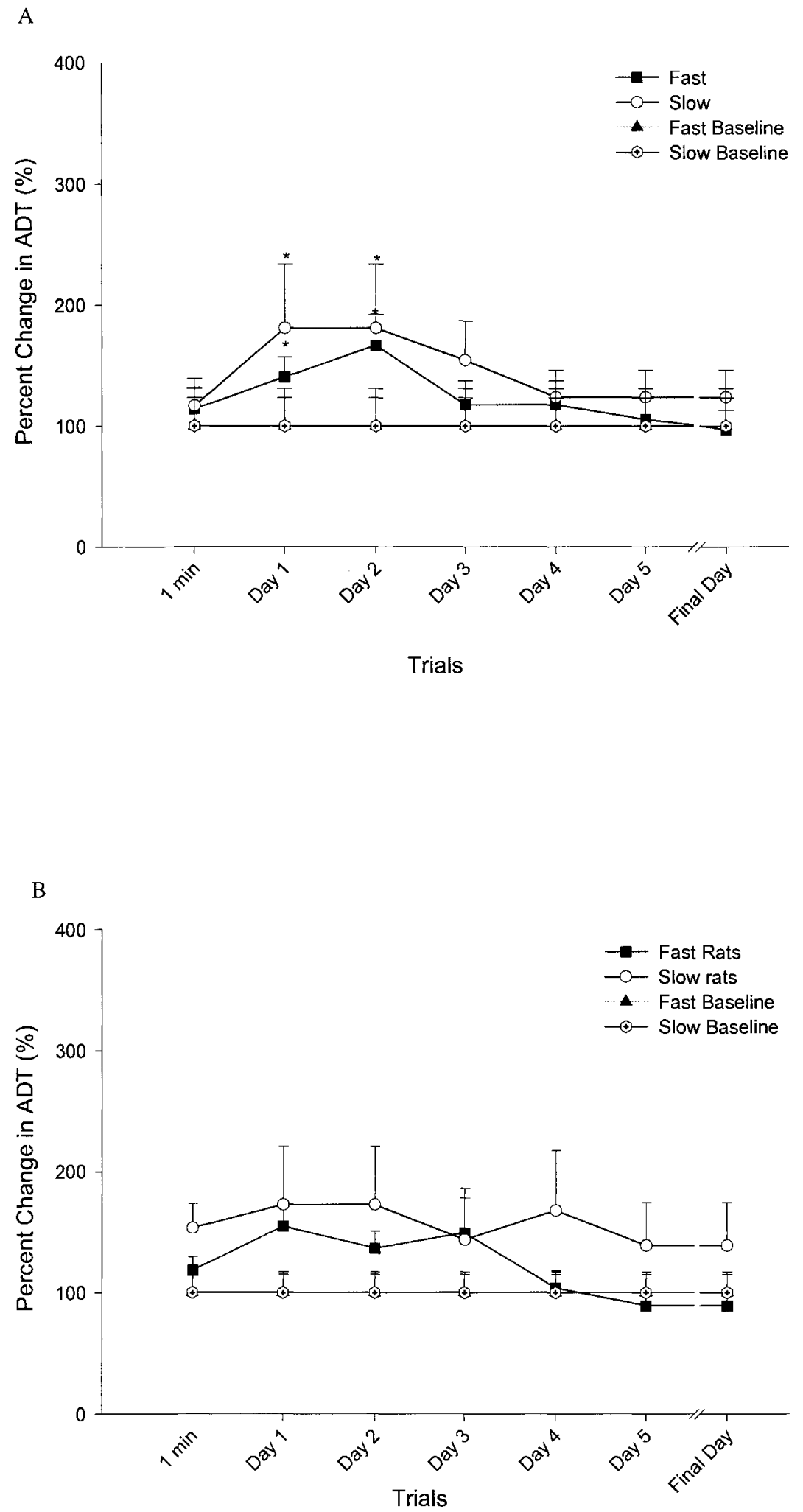
2) LFS administered to non-kindled left spanning area with ADT tested on kindled left amygdala:

After the application of LFS $(1 \mathrm{~Hz} ; 100 \mu \mathrm{A} ; 30 \mathrm{~s})$ to the spanning left amygdala electrode there was no significant effect on ADT as measured from the kindled left discrete amygdala electrode. Neither was there any main effect of strain. Effects over the daily repeated trials did not reveal any significant changes in ADT. The greatest percent change in ADTs for the FAST rats occurred on Day $3(116.0 \pm 35.7 \%)$ and on Day 1 for the SLOW rats $(117.0 \pm 87.3)$ (Figure 7 ). Furthermore, there were no significant strain, trial, or interaction effects upon ADTs, tested from the left amygdala discrete electrode, after the LFS $(1 \mathrm{~Hz} ; 100 \mu \mathrm{A})$ of the $90 \mathrm{sec}$ duration was administered to the electrode spanning the left amygdala. 
Figure 7 - Average ( \pm S.E.M.) percent change in afterdischarge thresholds (ADTs) following the application of the LFS $(1 \mathrm{~Hz} ; 100 \mu \mathrm{A} ; 30 \mathrm{~s})$ to the spanning electrode in the left amygdala with ADTs measured from the kindled amygdala electrode. 


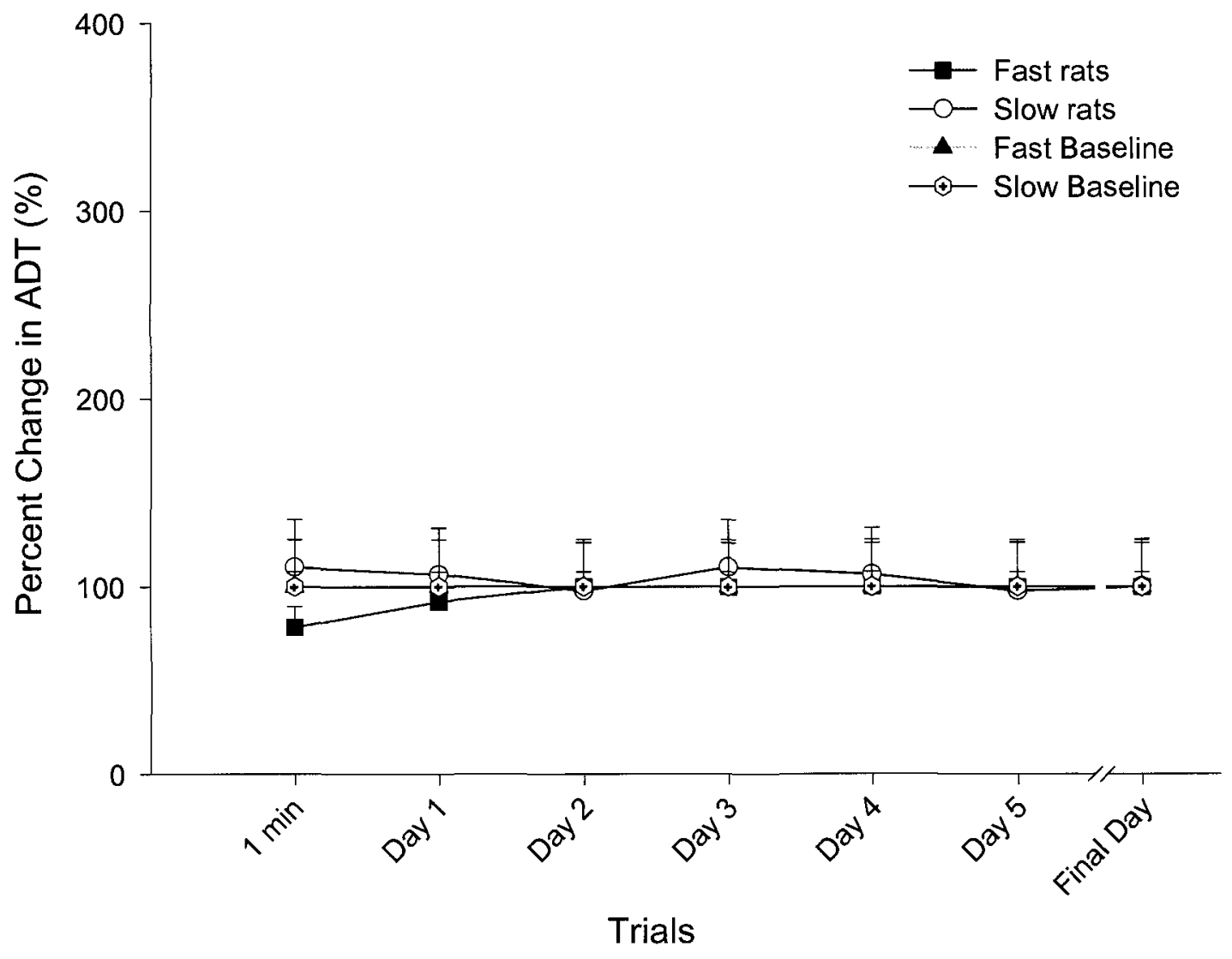


3) LFS administered to kindled left spanning area with ADT tested on kindled left spanning area:

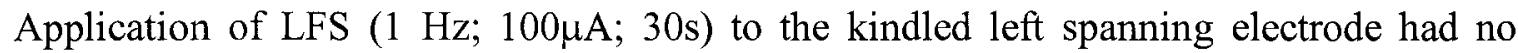
strain, trial and/or interaction effects upon the ADT, as was measured from this electrode. With a longer duration LFS $(1 \mathrm{~Hz} ; 100 \mu \mathrm{A} ; 90 \mathrm{~s})$ no significant strain effects and/or trial effects over the total number of days following the LFS were observed. However, the Newman-Keuls follow-up comparisons, which measured inter-trial intervals, showed a minimal effect $(p<0.08)$ from baseline to Day 3 and somewhat lager effect between days 2 and $6(\mathrm{p}<0.50)$

4) LFS administered to kindled left spanning area with ADT tested on the previously kindled discrete electrode:

There were no significant results obtained for percent change in the ADTs for this LFS manipulation.

\section{Histology}

Histological analysis showed that the electrode placements were in the targeted areas of the brain. The implantation sites were verified using light microscopy to determine the location of the discrete amygdala electrode being within the amygdaloid complex and the spanning electrode located in the amygdaloid-piriform area (Figure 8). In addition cresyl violet staining methods showed that any neuronal damage or lesioning that occurred was restricted to the electrode tip area. 
Figure 8 - A photomicrograph of the left basolateral amygdala of a FAST rat (Carys \#4) stained with Cresyl Violet showing necrosis around the point of insertion of a discrete electrode. 


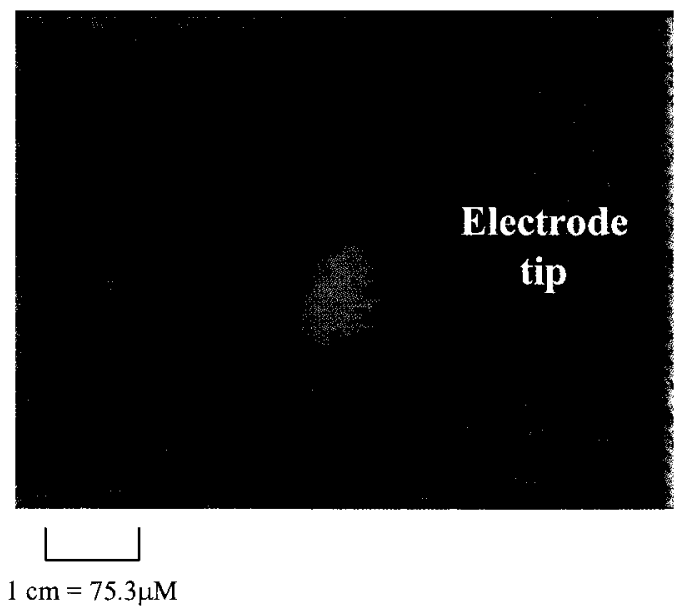




\section{EXPERIMENT 2 - LOW FREQUENCY STIMULATIONS APPLIED}

\section{CONTRALATERALLY}

\section{Animals}

Originally, 19 FAST and 20 SLOW male rats underwent the surgical procedure for electrode implantation; these animals were new subjects. Due to unexpected circumstances that occurred throughout the course of experimentation, 1 SLOW and 2 FAST rats were removed from the study due to a stroke, loss of a headplug assembly and a severe pain response on the kindled electrode. The animals were divided into the right amygdala kindled group, which consisted of 8 FAST and 9 SLOW rats and the left spanning kindled group, which was made up of 9 rats for each strain.

\section{Surgery}

The surgical protocol was identical to experiment 1 with the only differences being in the sites of electrode implantation. The discrete electrode was now placed in the right amygdala and the spanning electrode was implanted in a region spanning the left amygdala. The stereotaxic co-ordinates (Pellegrino et al., 1979) used for the electrode implantation for this experiment can be found in Appendix 1 and a schematic representation of the implanted electrodes can be seen in Figure 9. 
Figure 9 - Schematic representation, (Paxinos and Watson, 2005) of the electrode placements showing the targeted regions. The red circle corresponds to the ground electrode. The green circles correspond to the spanning electrode and the blue circles correspond to the amygdala electrode. The upper coronal section, at $2.0 \mathrm{~mm}$ anterior to bregma, represents the region where the first tip for the spanning electrode was inserted into the piriform area. The middle coronal section, at $0.0 \mathrm{~mm}$ bregma, represents where the electrode tip for the twisted, discrete electrode was placed into the amygdala complex. The bottom coronal section, at $2.0 \mathrm{~mm}$ posterior to bregma, represents where the second electrode tip for the spanning electrode was inserted within the amygdala/piriform cortex. The figure to the left shows where the electrodes were inserted in relation to the bregma on the rat skull. 


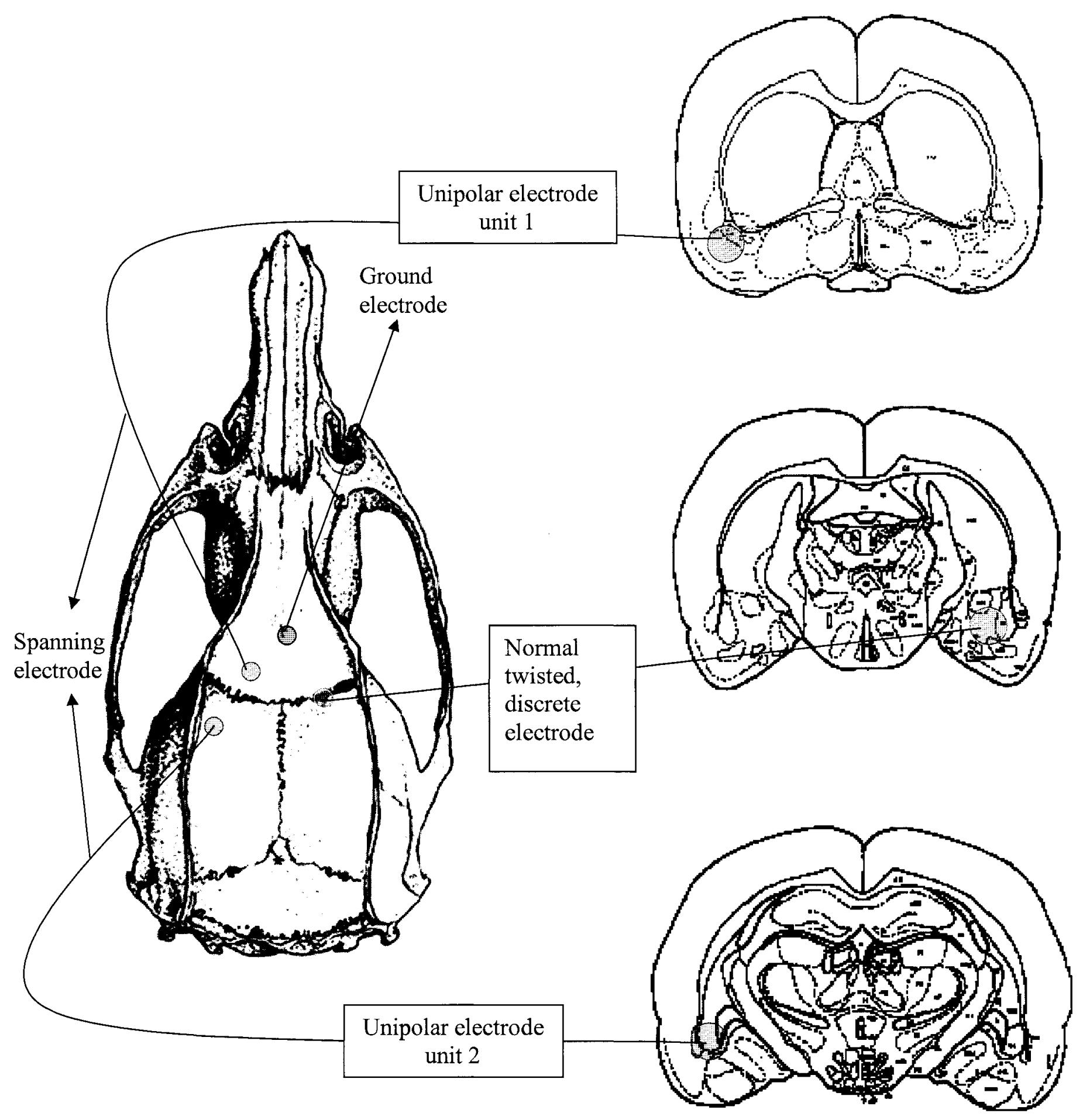




\section{LOW FREQUENCY STIMULATION MANIPULATIONS}

First, before the animals were separated into two experimental groups, the ADTs were found for both electrodes. While the first group was kindled on the right discrete amygdala electrode, the second group was kindled on the left spanning electrode. Once stable post-kindling ADTs were generated for each experimental condition, LFS manipulations were administered.

\section{Kindled right discrete amygdala electrode (Group 1)}

LFS $(1 \mathrm{~Hz} ; 100 \mu \mathrm{A})$ was first administered at $30 \mathrm{sec}$ duration to the kindled right amygdala via the discrete electrode. ADTs were measured from the same electrode 1 minute after the application of the electrical stimulation as well as daily until there was no variability in the value of the stimulus for at least three consecutive days. Once the ADTs were stable LFS $(1 \mathrm{~Hz} ; 100 \mu \mathrm{A})$ was applied at $90 \mathrm{sec}$ duration on the kindled discrete right electrode with ADTs measured from the right amygdala beginning 1 minute after application of the experimental stimulus and continuing daily until the stability of ADTs was achieved.

Following the aforementioned electrical manipulations upon the right discrete amygdala electrode, LFS $(1 \mathrm{~Hz} ; 100 \mu \mathrm{A})$ stimulations at $30 \mathrm{sec}$ and $90 \mathrm{sec}$ durations were then applied to the non-kindled spanning electrode in the left hemisphere and ADTs for both LFS durations were measured from the discrete electrode in the contralateral hemisphere using the procedure, described in the preceding paragraph. 


\section{Kindled left spanning electrode (Group 2)}

LFS $(1 \mathrm{~Hz} ; 100 \mu \mathrm{A} ; 30 \mathrm{~s})$ was administered to the kindled left spanning electrode with the ADTs being measured from the same electrode 1 minute after the application of the electrical experimental stimulus. ADTs were then measured daily until there was no variability in the value of the stimulus needed to elicit a convulsive seizure at least three consecutive days. Once the ADTs were stable another LFS test stimulus $(1 \mathrm{~Hz} ; 100 \mu \mathrm{A})$ was applied to the kindled left spanning electrode, but this time for $90 \mathrm{sec}$ duration. The ADTs were measured from the left spanning electrode beginning 1 minute after the application of the experimental stimulus and continuing daily until the stability of ADTs was achieved.

Following the electrical manipulations described above upon the left spanning electrode, LFS $(1 \mathrm{~Hz} ; 100 \mu \mathrm{A})$ administration at $30 \mathrm{sec}$ and $90 \mathrm{sec}$ durations were then applied to the non-kindled right amygdala discrete electrode. The ADTs for both LFS durations were measured from the spanning electrode in the left hemisphere using the procedure, described in the preceding paragraph.

\section{Histology and Data Analysis}

At the completion of all of the experimental manipulations, the animals were euthanized and their brains were subjected to the same histological procedures as outlined in experiment 1 . Also, the statistical treatment of the acquired data was carried out in a similar fashion to that described in Experiment 1. 


\section{RESULTS}

\section{Kindling Rates and Afterdischarge Thresholds}

The kindling rates showed a significant main effect of strain $\left(F_{1,16}=27.0, p<0.01\right)$ such that the SLOW rats had a significantly higher kindling rate than the FAST rats when the right amygdala was the primary site of kindling. The same trend was observed when the left spanning region was the primary site of kindling $\left(F_{1,14}=7.2, p<0.01\right)$ (Figure 10).

The ADTs showed no significant strain effect both in the pre- or post-kindling condition, regardless of the type of electrode used. However, both experimental conditions the right amygdala discrete electrode $(\mathrm{p}<0.0001)$ and the left spanning electrode $(\mathrm{p}<0.01)-$ demonstrated a significant decrease in ADT from the pre-kindling day to Day 1 (Figure 11).

However, there were no significant changes in the ADTs, as main effect of strain and/or trials, over the subsequent experimental days, following the initial reductions in the ADTs. This indicated that regardless of the experimental condition there was no variability in the ADTs in both the FAST and SLOW rats (Figure 11). 
Figure 10 - Average ( \pm S.E.M.) number of stimulations required to elicit the initial stage 5 seizure in the FAST and SLOW strains as a function of the kindling site (the right discrete amygdala vs. the left spanning region).

'*' = significantly higher kindling rates for the SLOW vs. the FAST rats via the right amygdala discrete electrode rats $\mathrm{p}<0.0001$.

' + ' = significantly higher kindling rates for the SLOW vs. the FAST rats via the left spanning electrode $\mathrm{p}<0.017$. 


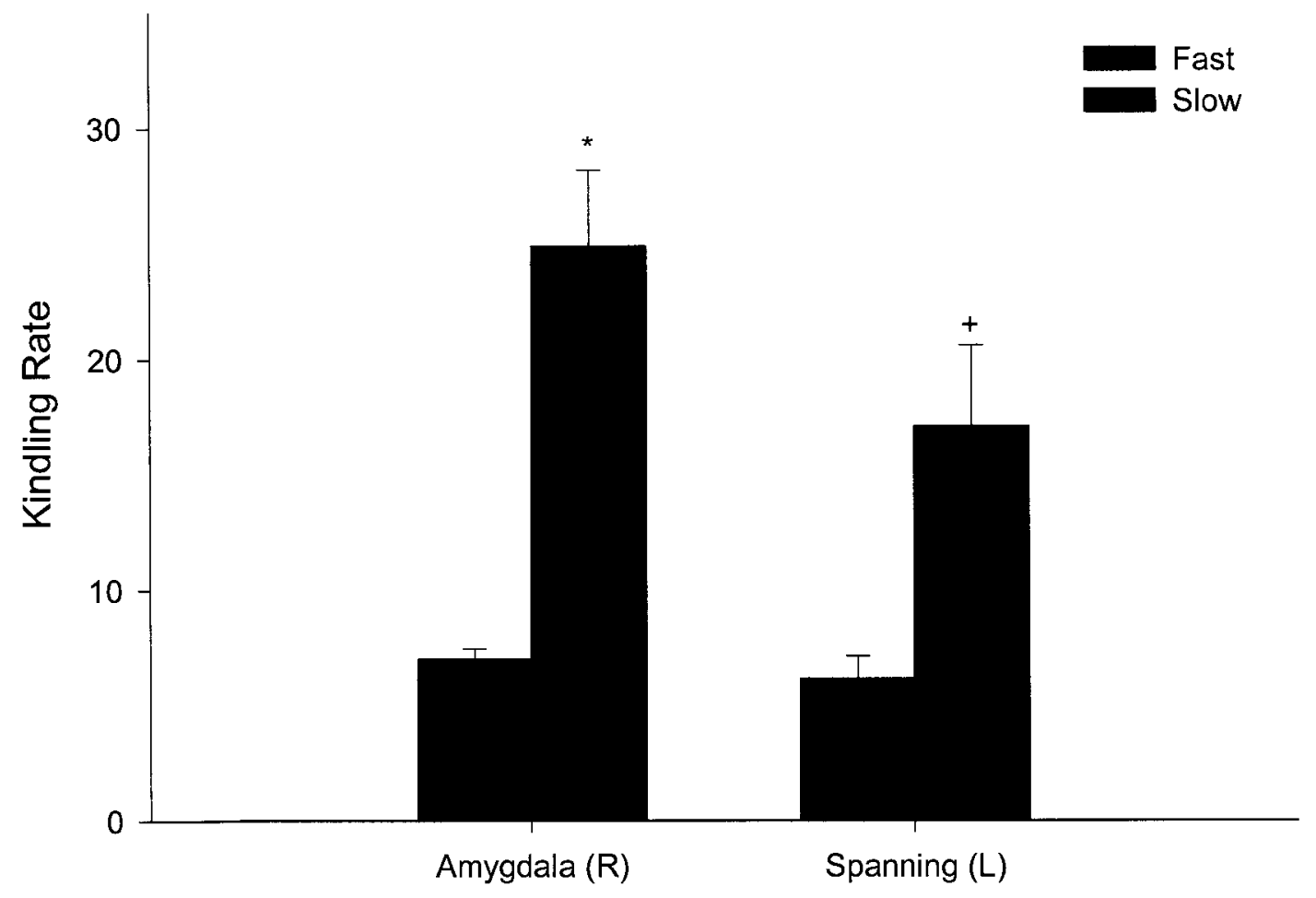


Figure 11 - Average ( \pm S.E.M.) percent change of ADTs of the FAST vs. the SLOW rats following the establishment of the kindled state through the right amygdala discrete electrode and the left spanning electrode.

' $*$ ' = significantly lower ADTs from the pre-kindled day to Day 1 for the right discrete amygdala, $\mathrm{p}<0.0001$.

'+' = significantly lower ADTs from the pre-kindled day to Day 1 for the left spanning electrode, $\mathrm{p}<0.0008$ 


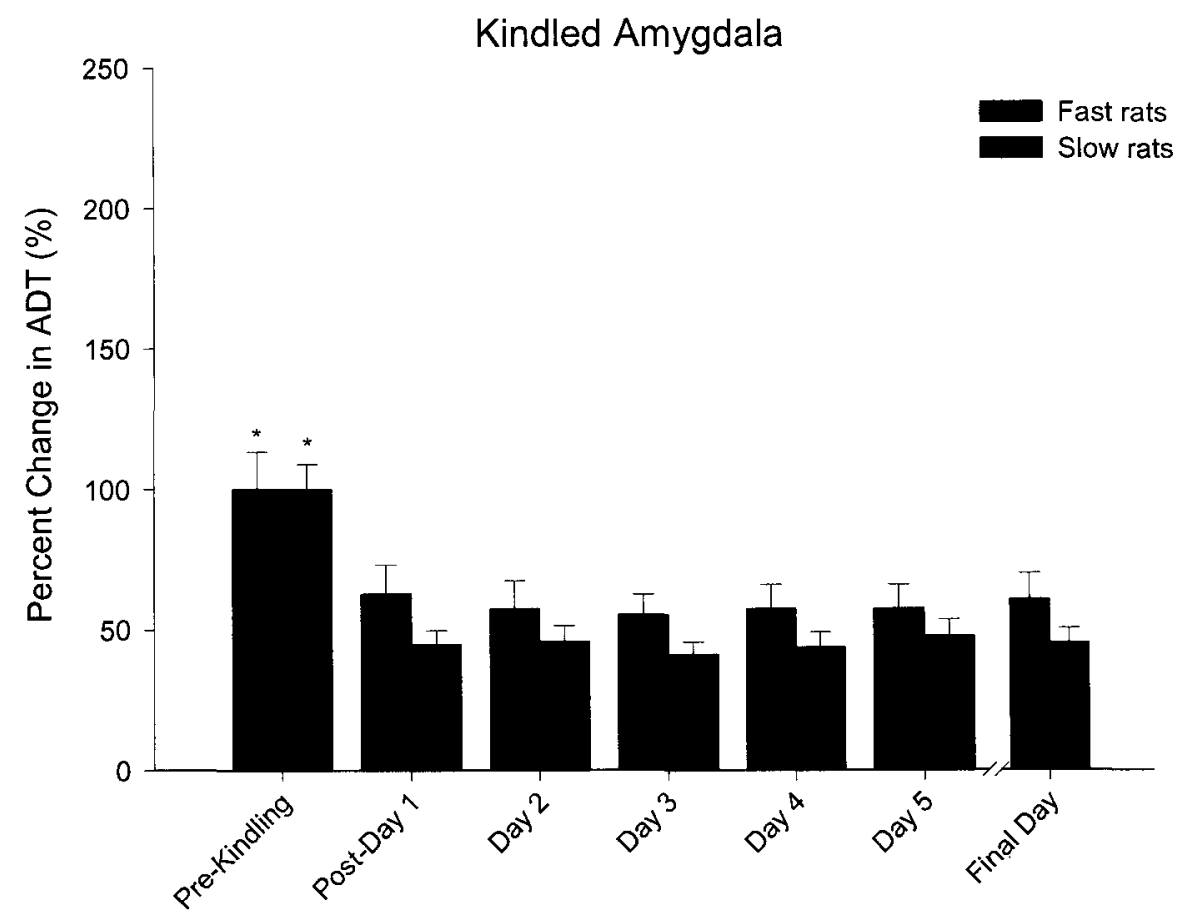

Trials

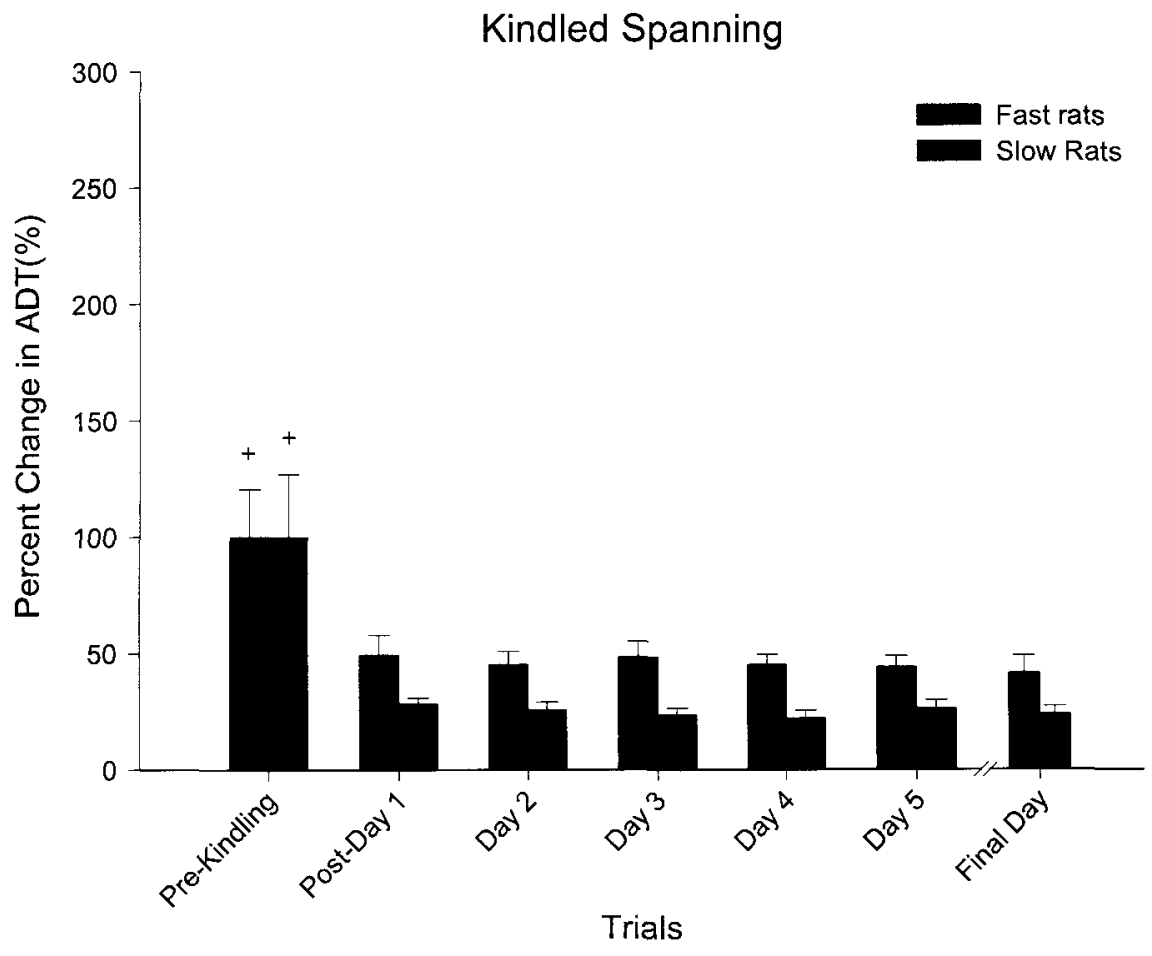




\section{LOW FREQUENCY STIMULATION MANIPULATIONS}

\section{Kindled right discrete amygdala electrode (Group 1)}

In the first LFS manipulation the electrical stimulus $(1 \mathrm{~Hz} ; 100 \mu \mathrm{A})$ was administered through the kindled right amygdala electrode for $30 \mathrm{sec}$ and the ADTs of the kindled right electrode was measured. There was a small significant strain effect in this experimental group ( $p>0.05$ ), as the SLOW rats exhibited a higher percent change in their ADTs compared with the FAST. These findings differ from those obtained in experiment 1, which didn't show any strain effect as a function of LFS. However, the difference between the FAST and SLOW rats was attributable to 3 SLOW rats whose thresholds were considerably higher compared with the other SLOW rats' thresholds, on the days following the application of the LFS. These three outliers had thresholds which were beyond the signal sensitivity settings of the oscilloscope with the upper measure of $1250 \mu \mathrm{A}$. Nevertheless, these data were included in the overall pool because over trials the ADTs of these animals became on par with the other subjects of the group.

There was a significant difference in the percent change of ADTs with respect to the trials $\left(\mathrm{F}_{1,7}=5.7, \mathrm{p}<0.0001\right)$ and follow-up comparisons at Newman-Keuls, $\alpha=0.05$ confirmed that these changes in the ADTs did, in fact, differ from Day 1 to Day 4 . The thresholds began to rise within 1 minute of the LFS with the greatest change occurring on Day $2(173.1 \pm 24.7 \%)$. Furthermore, the SLOW rats' thresholds $(151.4 \pm 30.4 \%)$ remained somewhat above the baseline (Figure 12A).

For the $90 \mathrm{sec}$ LFS $(1 \mathrm{~Hz} ; 100 \mu \mathrm{A})$ there were no significant effects with respect to strain. However, a definite trial effect $\left(\mathrm{F}_{1,7}=12.74, \mathrm{p}<0.01\right)$ was observed. Again, the 
thresholds began to rise immediately after the application of LFS and the greatest change in ADT $(273.9 \pm 37.9 \%)$ occurred on Day 1 following the administration of the stimulus. Thereafter, the ADTs of the two strains remained well above $(128.7 \pm 18.1 \%)$ the baseline value up to the final day (Figure 12B).

Applying the LFS $(1 \mathrm{~Hz} ; 100 \mu \mathrm{A})$ for the 30 and 90 second duration to the left spanning electrode with ADTs tested on the kindled right amygdala did not result in any significant alterations of the ADTs and neither main effect of strain or any of the interaction effect that involved these two variables (30s and 90s LFS) attained significance. 
Figure 12 - A) Average ( \pm S.E.M.) percent change in ADT in the time following application of a LFS (1 Hz; $100 \mu \mathrm{A} ; 30 \mathrm{~s})$ to the kindled amygdala with ADTs measured from the kindled discrete amygdala electrode.

B) Average ( \pm S.E.M.) percent change in ADTs in the time following application of a LFS $(1 \mathrm{~Hz} ; 100 \mu \mathrm{A} ; 90 \mathrm{~s})$ to the kindled amygdala with ADTs measured from the kindled discrete amygdala electrode. 
A

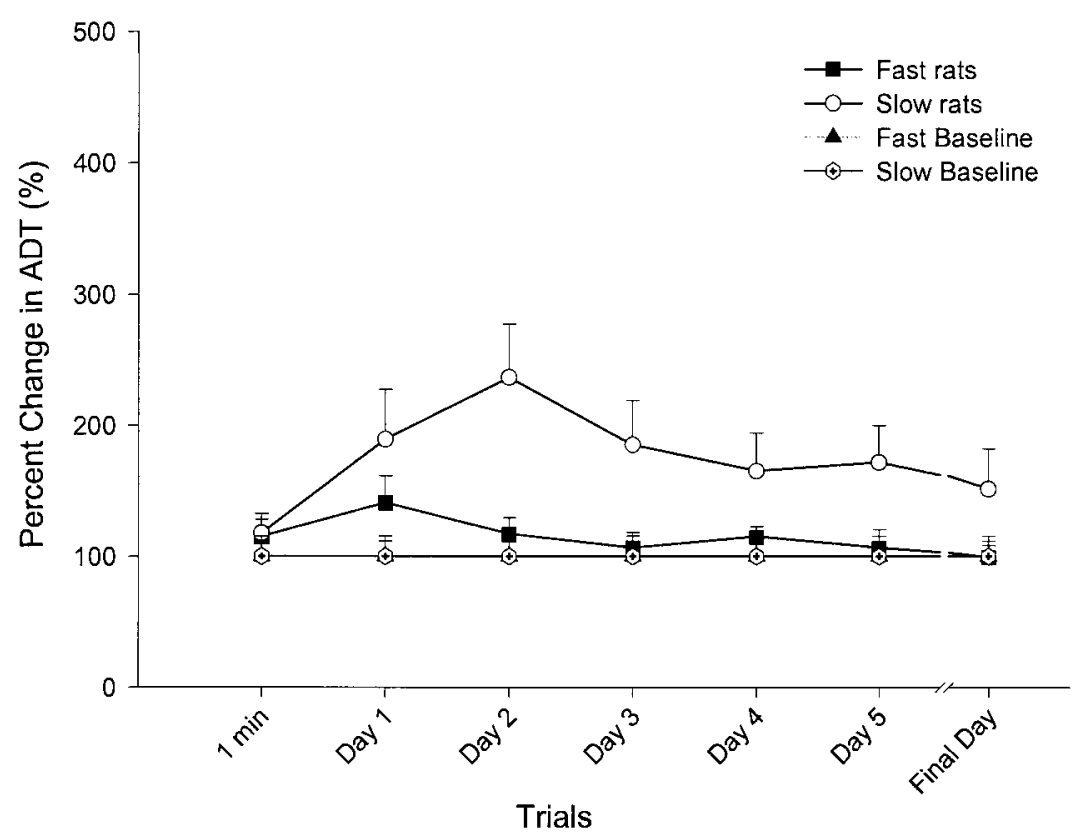

B

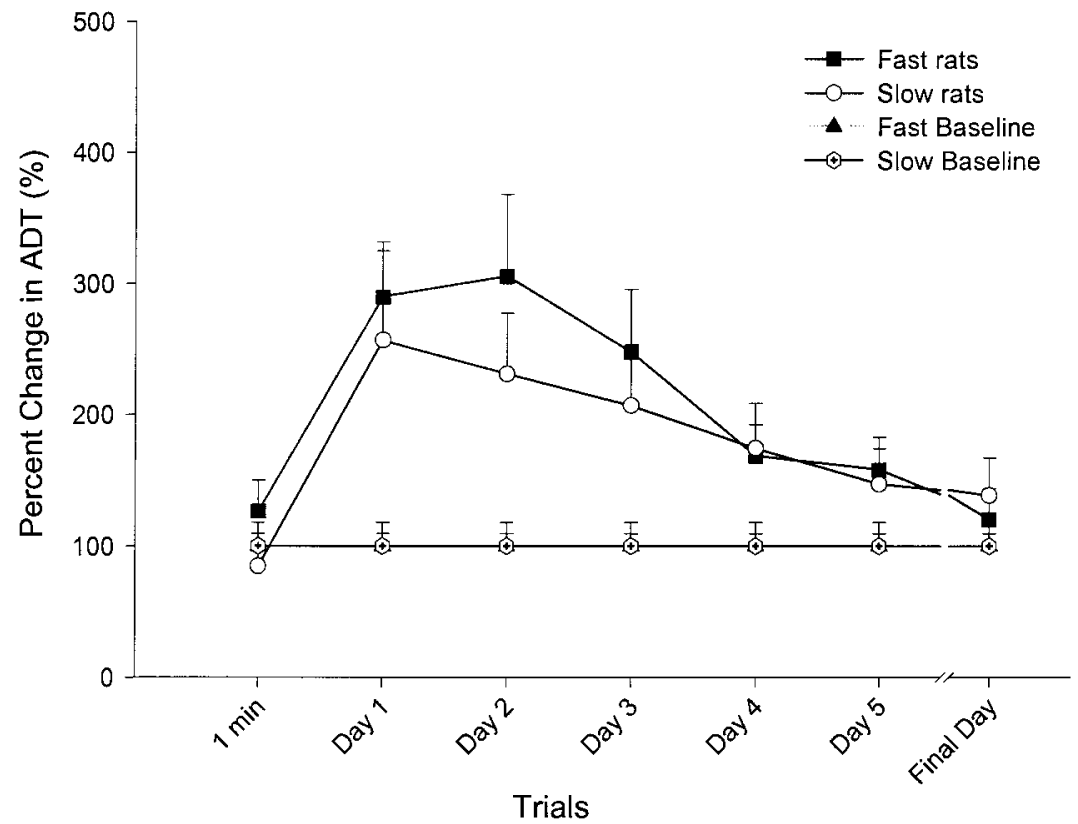

Reproduced with permission of the copyright owner. Further reproduction prohibited without permission. 


\section{Kindled left spanning electrode (Group 2)}

Applying LFS to the kindled spanning electrode, with the ensuing ADTs measured on the same electrode, produced no significant strain effects either for the $30 \mathrm{sec}$ or $90 \mathrm{sec}$ condition. However, there was a significant increase in the ADTs over the repeated trials for both the $30 \mathrm{sec}(\mathrm{p}<0.001)$ and the $90 \mathrm{sec}(\mathrm{p}<0.01)$ stimulation durations (Figure 13).

Applying LFS $(1 \mathrm{~Hz} ; 100 \mu \mathrm{A})$ to the right amygdala electrode at the 30 and 90 second durations, with ADTs tested on the kindled left spanning electrode, did not result in any significant changes in the ADTs. Neither main effect of strain nor of the experimental trials attained significance. 
Figure 13 - Average ( \pm S.E.M.) percent change in the ADTs in the time following the application of LFS $(1 \mathrm{~Hz} ; 100 \mu \mathrm{A})$ at $30 \mathrm{sec}$ and $90 \mathrm{sec}$ durations to the kindled spanning electrode with the ADTs measured from the same electrode. 


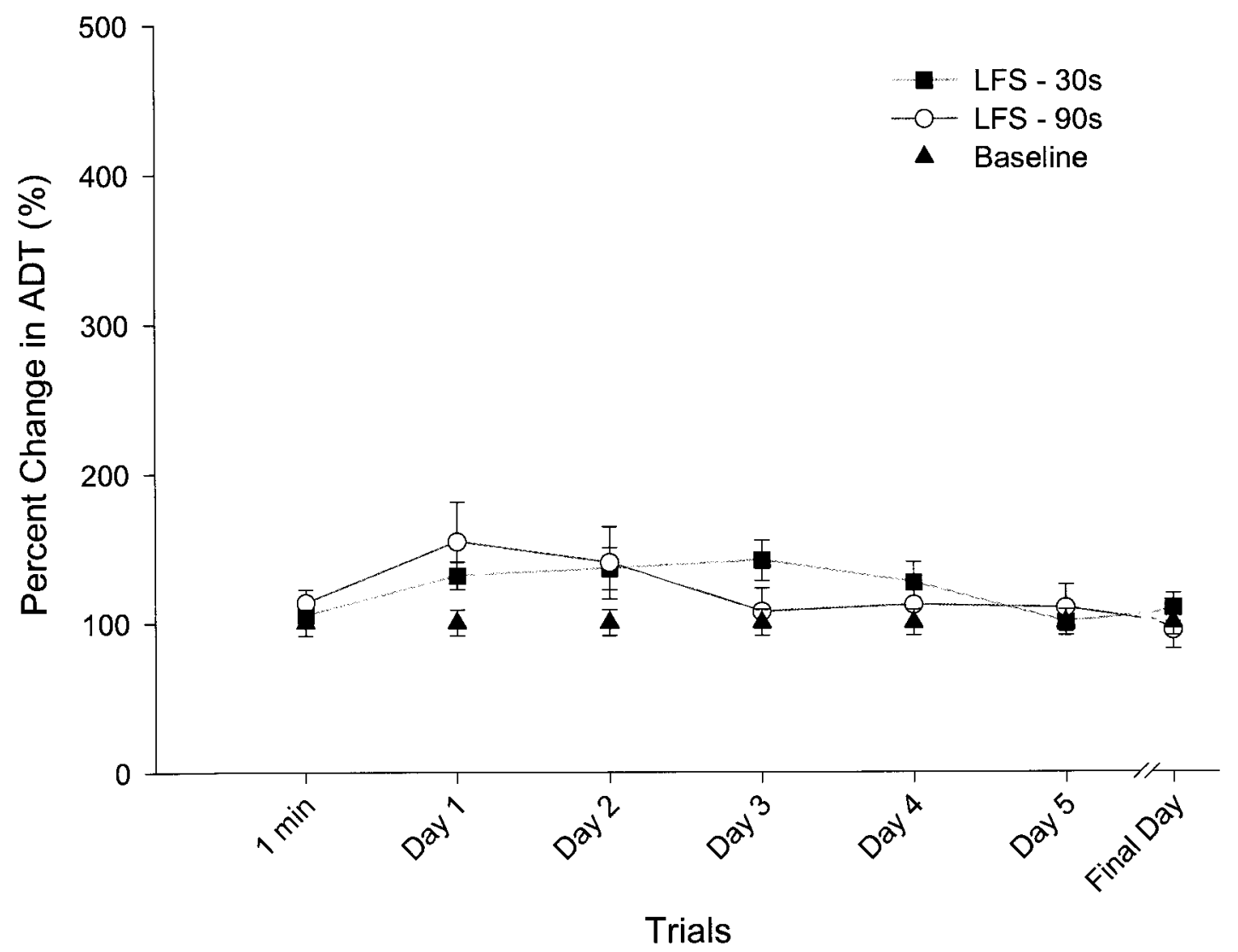




\section{Histology}

Results of cresyl violet histological staining verified that electrodes were implanted in the targeted brain regions. There was little pathology around the electrode tip and this was attributed to cell death occurring due to implantation. No other areas within the limbic system showed any gliosis or necrosis. 


\section{EXPERIMENT 3 - EFFECT OF ALTERING LFS PARAMETERS}

\section{Animals}

The previous experiments showed little or no strain difference between the FAST and SLOW rats with respect to the effects of LFS, regardless of the duration. Hence, only FAST rats $(\mathrm{N}=10)$ were used for this study, primarily due to their faster kindling rates; these animals were naïve to the protocol. Due to the loss of headplug assemblies two rats were removed from the study.

\section{Surgery}

The overall surgical protocol was identical to the preceding experiments, with the exception that the electrodes were inserted only in the right hemisphere (Figure 14). The stereotaxic co-ordinates used (Pellegrino et al., 1979) can be found in Appendix 1. The spanning electrode used in this experiment was slightly modified in that $2 \mathrm{~mm}$ of the Diamel insulation at the electrode tip was removed prior to the implantation (Figure 15). This allowed a greater surface area of the tip of the spanning electrode to be in contact with the brain tissue. 
Figure 14 - Schematic representation, (Paxinos and Watson, 2005) of the electrode placements showing targeted regions. The red circle corresponds to the ground electrode. The blue circle corresponds to the amygdala electrode and the green circles correspond to the spanning electrode. The upper coronal section, at $2.0 \mathrm{~mm}$ anterior to bregma, represents the region where the first tip for the spanning electrode was inserted into the piriform area. The middle coronal section, at $0.0 \mathrm{~mm}$ bregma, represents the electrode tip for the twisted, discrete electrode placed into the amygdaloid complex. The bottom coronal section, at $2.0 \mathrm{~mm}$ posterior to bregma, represents where the second electrode tip for the spanning electrode was inserted within the amygdala/piriform cortex. The figure to the left shows the insertion points of the electrodes in relation to the bregma on the rat skull 


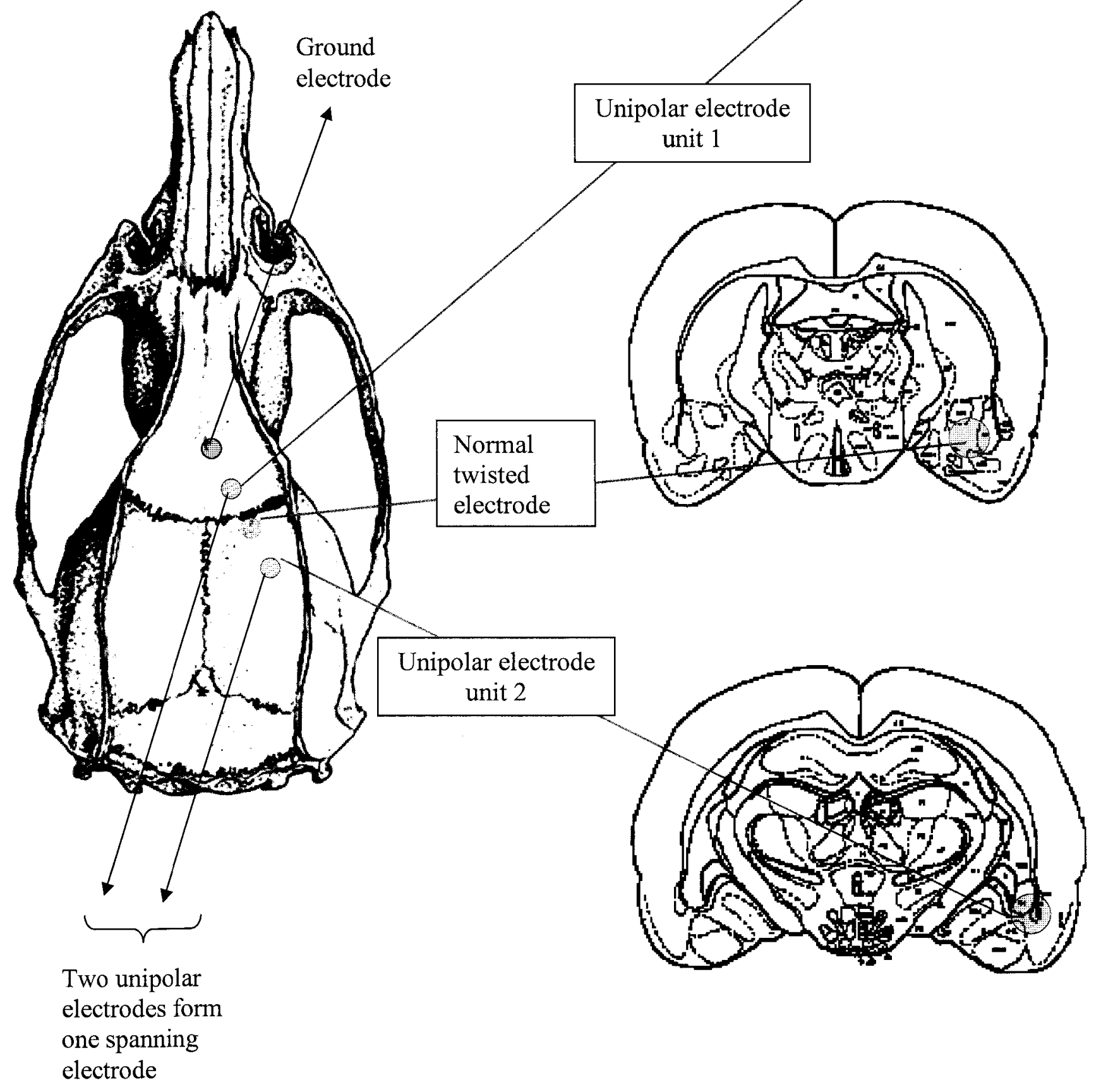


Figure 15 - Schematic representation of the modified spanning electrode. 


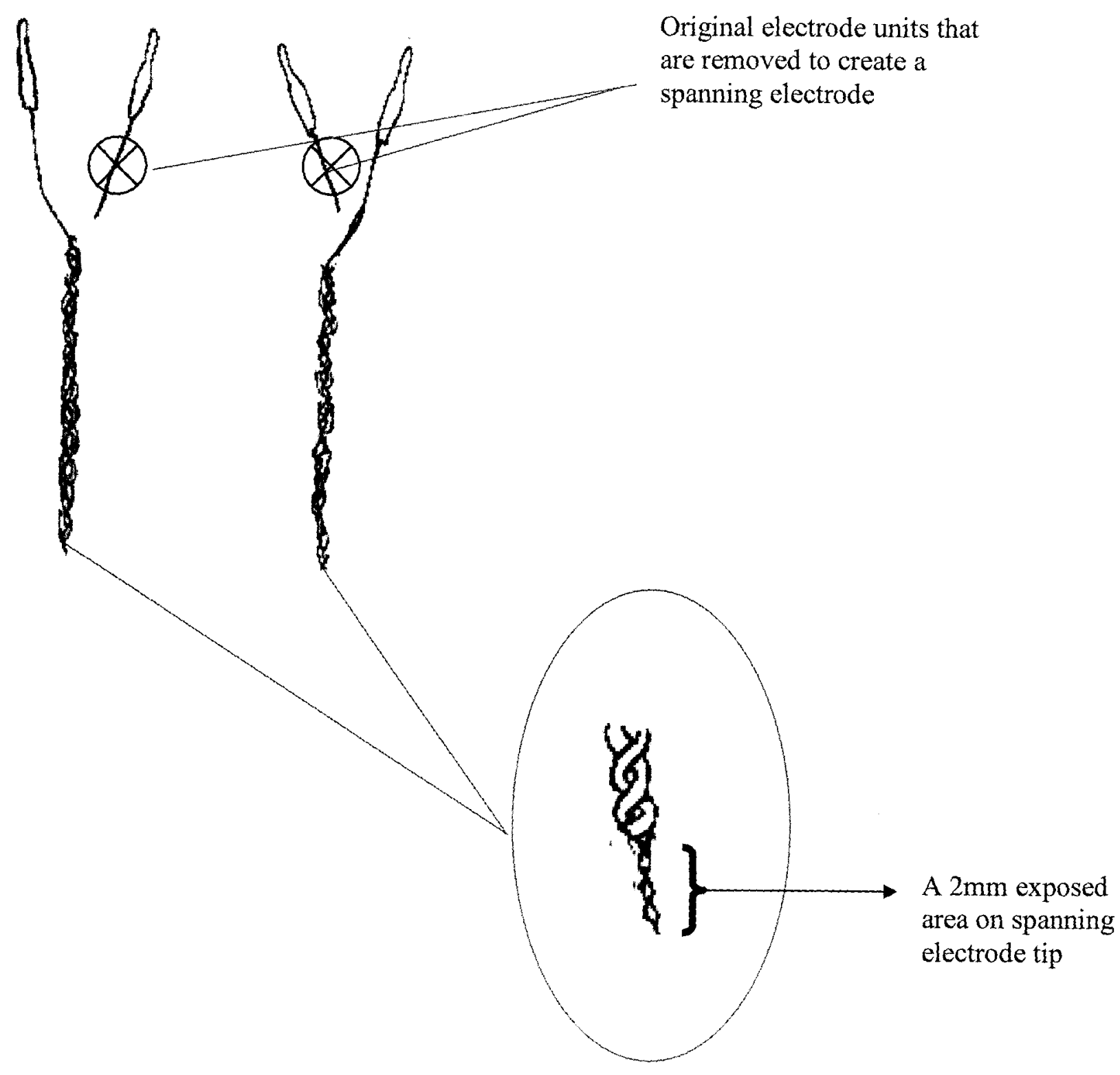

Reproduced with permission of the copyright owner. Further reproduction prohibited without permission. 


\section{LOW FREQUENCY STIMULATION MANIPULATIONS}

As in the previous two experiments LFS was administered following kindling. The kindling methodology used in this experiment was identical to the others, with the exception that only the discrete electrode was used. Also, in lieu of the fact that the $90 \mathrm{sec}$ duration LFS $(1 \mathrm{~Hz} ; 100 \mu \mathrm{A})$ produced significantly higher percent increases in the ADTs compared with the $30 \mathrm{sec}$ duration, the longer duration LFS was used.

\section{Low Frequency Stimulation - intensity and duration manipulations}

To replicate the effects of LFS upon the ADTs as seen in experiment 1, the electrical stimulation was first administered to the discrete amygdala electrode and ADT measurements were taken from the same electrode. The three subsequent LFS manipulations were all done via the spanning electrode with intensity of the stimulus being changed to 150,300 and 600 $\mu \mathrm{A}$, respectively. In addition, in the fourth treatment the intensity of the LFS stimulus was changed to $300 \mu \mathrm{A}$ and its duration to 300 seconds.

\section{Histology}

At the completion of the experiment the animals were perfused and the brains were sectioned and stained with cresyl violet. Also, as the histology for the previous experiments showed no abnormal necrosis, another stain fluoro-jade B, was used to determine if there was any neuronal degeneration after the LFS. The fluoro-jade B protocol involved passing the dried, mounted coronal sections through a series of steps in the following order: I. submersion in $100 \%$ absolute ethanol for 3 minutes; II. submersion for 1 minute in each of the following - $70 \%$ ethanol, $30 \%$ ethanol and distilled water; III. incubation in $0.06 \%$ 
potassium permanganate for 15 minutes at room temperature; IV. brief rinse in distilled water; V. incubation in $0.01 \%$ fluoro-jade B solution for 30 minutes at room temperature; VI. Rinse thrice in distilled water; VII. removal of excess water on slides; VIII. immersion in clearene and coverslipped with D.P.X. The fluorojade-B stained sections were examined under a Leica fluorescent microscope using the fluorescein isothiocyanate (FITC) filter.

\section{RESULTS}

\section{Low frequency stimulation at $100 \mu \mathrm{A}$ administered to discrete and spanning electrodes}

Application of the LFS $(1 \mathrm{~Hz} ; 100 \mu \mathrm{A} ; 90 \mathrm{~s})$ applied to the kindled amygdala, with ADTs measured off the discrete electrode, produced large elevations in the ADTs within 1 minute following the administration. The highest threshold percent increase $743.8 \pm 256.0 \%$ occurred on Day 4. Thereafter, the ADTs progressively decreased towards the baseline value, although always remaining somewhat above it $(398.3 \pm 137.8 \%)$ (Figure 16).

There was a significant trial effect $(p<0.0001)$ throughout the experiment. It is important to mention that two animals had thresholds, which were considerably greater than those of the other subjects, thereby introducing additional error on Day 2 through to Day 5. These animals were, nevertheless, kept within the subject pool as their ADTs sufficiently decreased over time (Figure 16).

LFS (1Hz; $100 \mu \mathrm{A} ; 90 \mathrm{~s})$ administered to the spanning electrode with ADTs measured from the discrete amygdala electrode produced similar effects. However, the findings were not as great as those obtained when LFS was delivered to the discrete amygdala electrode. Surprisingly, initial ADTs within the first minute of the electrical stimulation $(66.3 \pm 13.8 \%)$ were below the baseline values, but within twenty-four hours following LFS they were above 
it. The greatest positive percent change in ADT occurred on Day $2(150 \pm 44.8 \%)$ (Figure 16).

In comparison, the first LFS treatment condition (discrete to discrete) was significantly different $(\mathrm{p}<0.01)$ from the second (spanning to discrete) (Figure 16). The similarities between the treatments was in terms of the variability of the ADT percent change over trials being significant $(\mathrm{p}<0.001)$. The follow-up comparisons with Newman-Keuls set at $\alpha=0.05$ showed that this variation was greater for the first treatment $(p<0.0006)$ than the first (Figure 16). 
Figure 16 - Average ( \pm S.E.M.) percent change ADTs in the time following application of a LFS $(1 \mathrm{~Hz} ; 100 \mu \mathrm{A} ; 90 \mathrm{~s})$ to Treatment 1: the kindled amygdala with ADTs measured from the kindled discrete amygdala electrode.

Treatment 2: the spanning electrode with ADTs measured from the kindled discrete amygdala electrode.

( **" = significantly higher ADTs in Treatment 1 than Treatment 2 $\left.\mathrm{F}_{1,12}=8.182, \mathrm{p}<0.014\right)$ 


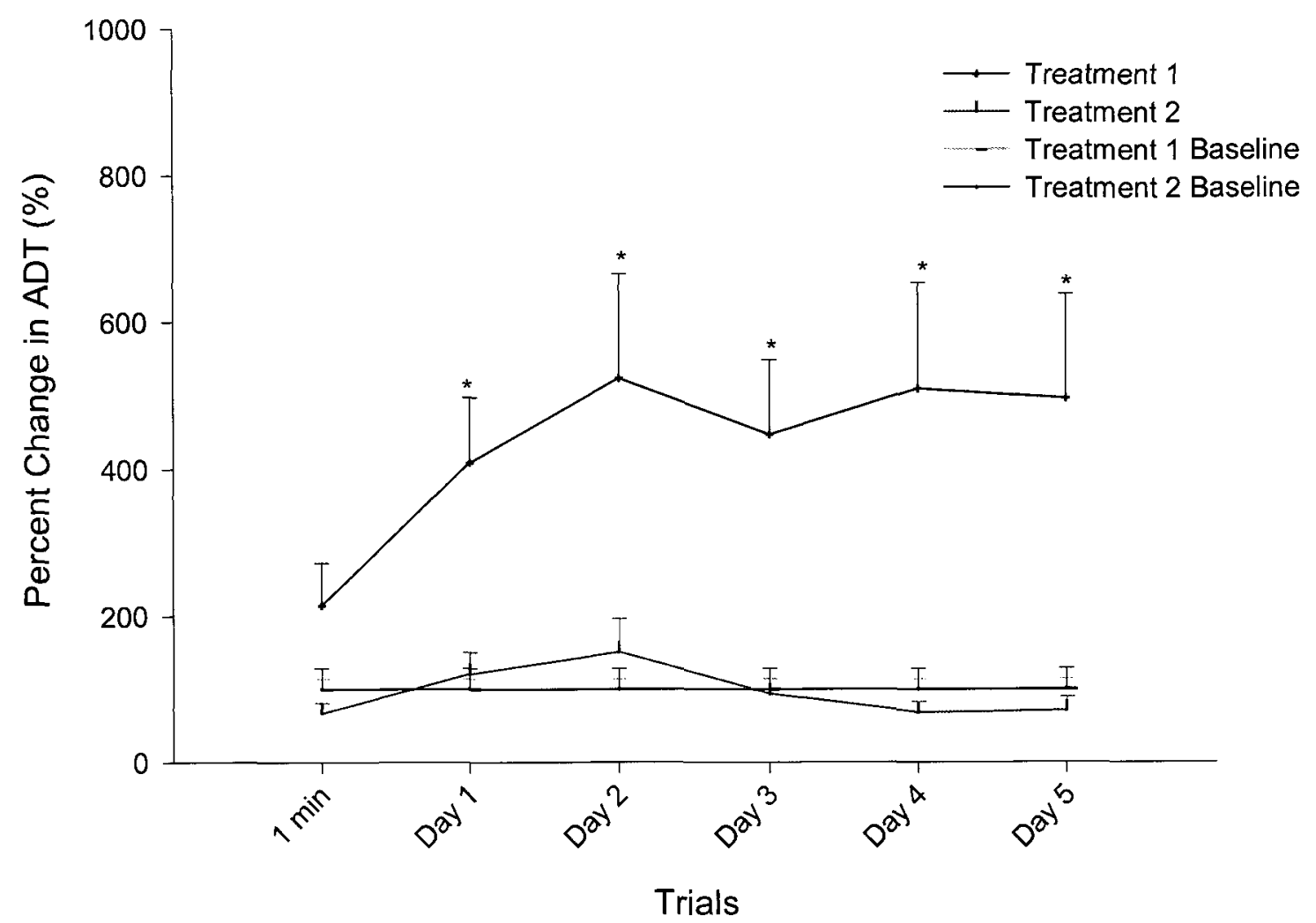




\section{Low frequency stimulation at varying intensities administered to the spanning electrode}

Altering the intensity of LFS had minimal effects on the percent change of ADT when applied to the spanning electrode. In fact, thresholds actually went below baseline values on most days following the application of the test stimulus. Analysis showed no significant differences between the three LFS treatments $(150,300,600 \mu \mathrm{A})$. Neither were the trial effects significant.

\section{Low frequency stimulation at long duration and moderate intensity}

When both the intensity and duration of LFS were altered to $300 \mu \mathrm{A}$ and 300 s, respectively and applied to the spanning electrode, the ADT percent changes were extraordinary. There was a significant trial effect $(p<0.001)$ from Day 1 through to Day 7 being significantly different $(\mathrm{p}<0.05)$ from the baseline ADT value (Figure 17).

ADTs initially dropped 1 minute following LFS but within 24 hours there was a large increase in the ADTs with a percent change of $415.4 \pm 171.7 \%$. The thresholds steadily rose and peaked on Day $4(633.3 \pm 151.6 \%)$ and then gradually decreased to $174.3 \pm 70.0 \%$, somewhat above the baseline value (Figure 17). As the large error bars indicate, there was a high degree of variability in the ADT percentage change between the individual subjects. The substantial changes in the ADTs were unprecedented by virtue of the fact that previously only LFS manipulations administered to the kindled amygdala through a discrete electrode demonstrated highly significant results. 


\section{Low frequency stimulation and mild-status effect}

The extraordinary increase in ADT was not the only unexpected result with the application of this treatment. Within 30 minutes after LFS stimulation all but one of the rats demonstrated a series of spontaneous seizures. On average $2.87 \pm 0.39$ seizures within the full hour of EEG recording was observed. One animal had 4 spontaneous fully-blown convulsive events within the 30 minutes. The rat that did not exhibit spontaneous seizures at within the first hour did so 24 hours later.

\section{Low frequency stimulation and seizure suppression}

Added to this was the fact that after the spontaneous seizures all of the rats had, to various degrees, shown "convulso-suppressive" effects. On stimulating the animals at their ADT values, the well-established relation between ADT and stage 5 seizure activity in fullykindled rats, was challenged in that a large portion of the subjects did not exhibit fully evolved convulsions.

This "convulso-suppressive" effect was seen in all of the treatment conditions in experiment 3 . However, only when LFS of $300 \mu \mathrm{A}$, administered at $300 \mathrm{sec}$ duration was used, was this effect more prominent. In the other cases, this phenomenon was rather sporadic and to a considerably lower extent. In fact, when LFS of longer duration was compared to the other LFS treatments in experiment 3 , there was a significantly $(p<0.05)$ higher incidence of seizure failure than in all the other treatments when ADT-equivalent stimulations were applied (Figure 18). 
Figure 17 - Average ( \pm S.E.M.) percent change ADTs in the time following application of LFS $(1 \mathrm{~Hz} ; 300 \mu \mathrm{A} ; 300 \mathrm{~s})$ to the spanning electrode with ADTs measured from the kindled discrete.

( $* * "$ = significantly higher ADTs than baseline, $\mathrm{p}<0.003$ ) 


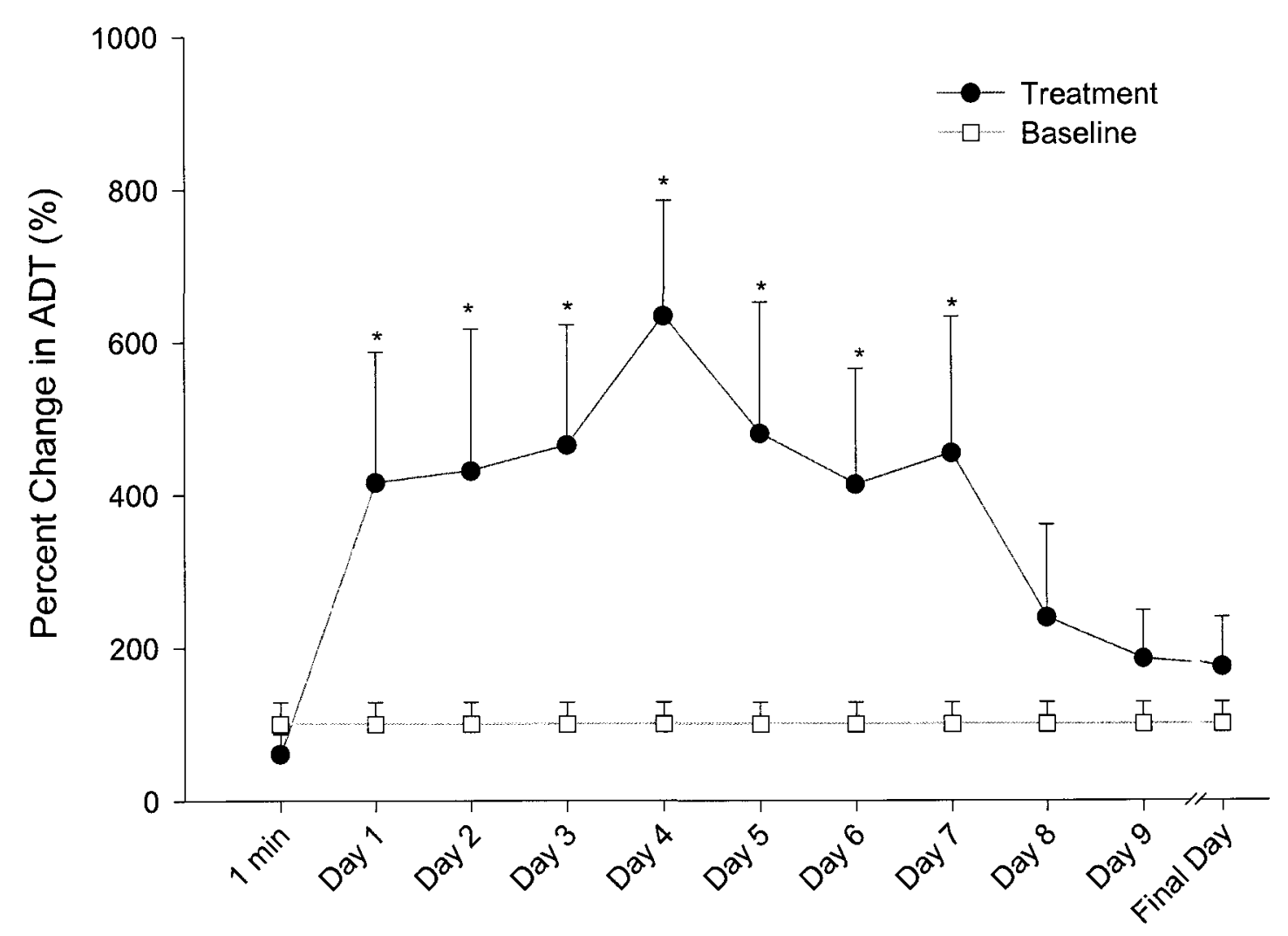

Trials 
Figure 18 - Average ( \pm S.E.M.) percent of seizure failure after the application of LFS in the following treatment conditions:

Treatment 1: LFS $(1 \mathrm{~Hz} ; 100 \mu \mathrm{A} ; 90 \mathrm{~s})$ applied to the kindled amygdala with ADTs measured from the kindled discrete amygdala electrode.

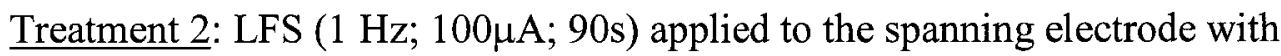
ADTs measured from the kindled discrete amygdala electrode.

Treatment 3: LFS (1 Hz; $150 \mu \mathrm{A} ; 90 \mathrm{~s})$ applied to the spanning electrode with ADTs measured from the kindled discrete amygdala electrode.

Treatment 4: LFS $(1 \mathrm{~Hz} ; 300 \mu \mathrm{A} ; 90 \mathrm{~s})$ applied to the spanning electrode with ADTs measured from the kindled discrete amygdala electrode.

Treatment 5: LFS $(1 \mathrm{~Hz} ; 600 \mu \mathrm{A} ; 90 \mathrm{~s})$ applied to the spanning electrode with ADTs measured from the kindled discrete amygdala electrode.

Treatment 6: LFS $(1 \mathrm{~Hz} ; 300 \mu \mathrm{A} ; 300 \mathrm{~s})$ applied to the spanning electrode with ADTs measured from the kindled discrete amygdala electrode.

'*'Treatment 6 was significantly different from all the other treatments $(\mathrm{p}<0.006)$ 


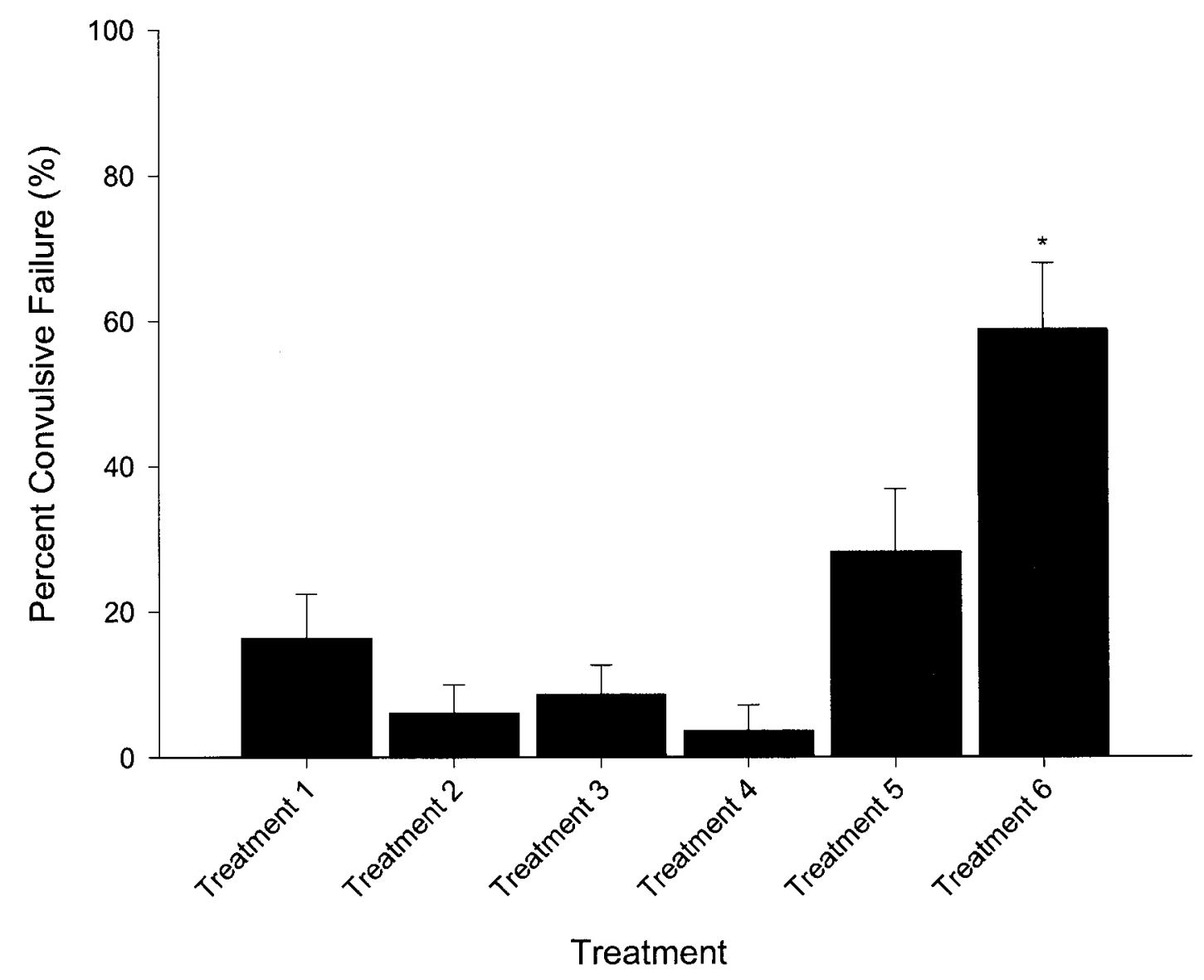




\section{Histology}

The generation of spontaneous seizures in the last treatment indicated that there might be some grave pathology within the brain. The spontaneous seizures observed in the experiment were akin to those commonly seen in animal models of status epilepticus which cause severe pathology in certain brain structures, especially the pyriform cortex, CA1 and CA3 regions of the ipsilateral hippocampus. However, both cresyl violet and fluorojade-B staining showed minimal necrosis around the electrode tip and that was attributed to electrode implantation (Figure 19). There was also no damage to the piriform cortex nor to the CA1 and CA3 region of the hippocampus. 
Figure 19 - Photomicrograph montage of histological staining of coronal sections after completion of LFS protocol showing:

A. Lack of pathology in the right pyriform cortex as shown with cresyl violet

B. Lack of pathology in the CA1 region of the hippocampus as shown with cresyl violet

C. Lack of pathology in the right pyriform cortex with as shown with fluorojade B stain

D. Small population of fluorojade B positive cells located around the electrode tip in the right basolateral amygdala nucleus.

E. Lack of pathology in the CA1 region of the hippocampus with Fluorojade B

F. Lack of pathology in the CA3 region of the hippocampus with Fluorojade B 

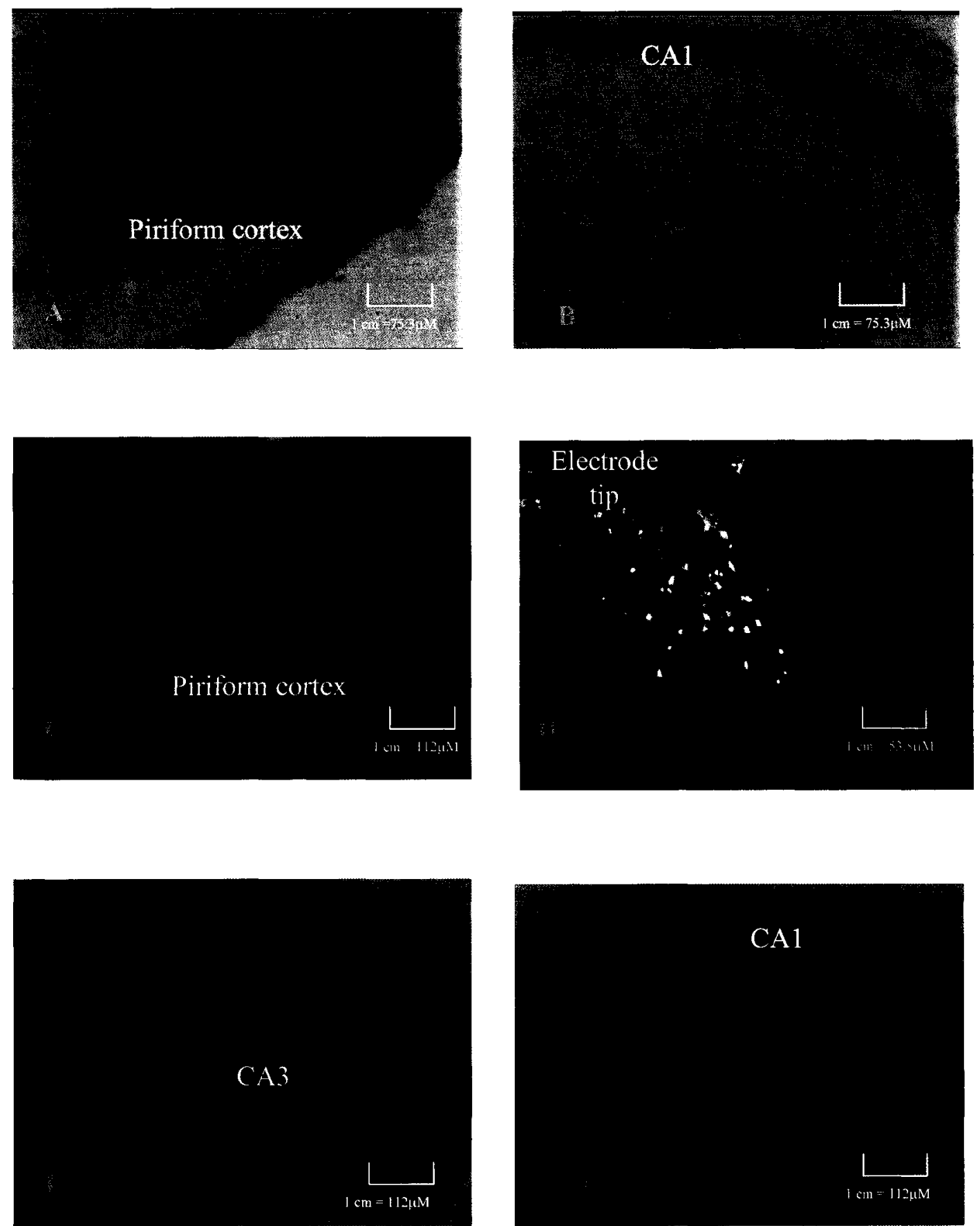


\section{EXPERIMENT 4: TRIGGERING SPONTANEOUS SEIZURES WITH LOW} FREQUENCY STIMULATION

In experiment 3 the application of LFS $(1 \mathrm{~Hz})$ of a moderate intensity $(300 \mu \mathrm{A})$ and long duration $(300 \mathrm{sec})$ not only resulted in the elevated ADT percent changes from their baseline values but also evoked a state of spontaneous convulsive seizures in all test subjects. Also, analysis of the effects of LFS at different intensities and durations in the previous experiment, demonstrated that the duration of a LFS stimulus might have been the more essential property in the generation of a mild-status effect. Therefore, the purpose of the present experiment was to further investigate this hypothesis.

\section{Animals:}

Twelve previously un-tested animals were used in the study. To determine if there was a strain effect of the "mild-status" state FAST $(n=6)$ and SLOW $(n=6)$ rats were used. In the second segment of the experiment the number of FAST and SLOW animals was reduced to 5 and 2, respectively, due to the loss of headplug assemblies.

\section{Surgery}

The surgical protocol was identical to that used in experiment 3 (see Figure 14) and the stereotaxic co-ordinates utilized in the present study can be found in Appendix 1 under the entry for experiment 4. 


\section{LOW FREQUENCY STIMULATION MANIPULATIONS}

All the rats were kindled on the discrete electrode located in the right amygdala. The study consisted of two LFS manipulations, following the stabilization of the ADTs. First, LFS $(1 \mathrm{~Hz} ; 300 \mu \mathrm{A} ; 300 \mathrm{~s})$ was applied to the kindled discrete electrode, with the ensuing ADTs being measured from the same electrode. Second, LFS $(1 \mathrm{~Hz} ; 300 \mu \mathrm{A} ; 300 \mathrm{~s})$ was administered to the spanning electrode and the thresholds were obtained from the discrete electrode in the kindled right amygdala. In both treatment conditions, the effects of LFS upon the ADTs were assessed 1 minute, $24 \mathrm{hrs}$ and every day thereafter, following the LFS stimulation.

\section{Histology}

At the completion of the experiments, the animals were sacrificed and the brain tissue preparations were stained with cresyl violet and fluoro-jade B to examine them for correct electrode placements and any neuropathology that might have occurred as a result of the insertion of the electrodes and/or experimental manipulations. The staining protocol used in the study was the same as in Experiment 3.

\section{RESULTS}

\section{Low Frequency Stimulation Manipulations}

1) LFS administration to kindled right amygdala with ADT tested on kindled right amygdala

LFS produced no significant effect of strain in the elevation of the ADTs. Therefore, the data were collapsed over the trials for statistical analysis, which produced a significant effect $\left(F_{1,9}=11.8, p<0.0001\right)$. The ADTs showed increases after the administration of the 
LFS, with the resultant percent change in the ADTs in this test condition being higher than those observed with the shorter duration stimulus in the earlier experiments (Figure 19). Within one minute of the LFS, ADTs rose by $481.0 \pm 154.3 \%$ of baseline values and continued rising until they peaked on Day 3 at $1151.0 \pm 157.1 \%$, well above baseline. The thresholds began declining but still stayed above baseline throughout the trials and finally stabilized at 552.4 185.2 (Figure 20). The high standard error was due to the high percentage of experimental subjects whose thresholds increased well above the calibration capacity of the oscilloscope (Table 1 ).

Unlike in experiment 3, all the animals did not exhibit spontaneous seizures. Only 2 FAST and 1 SLOW rat developed recurring spontaneous seizure activity on the day of the test stimulation. However, all the rats did show spindle activity and burst of seizure discharges after LFS administration (Table 2). There was a $13.2 \%$ and $1.6 \%$ seizure failure in the FAST and SLOW animals, respectively.

This indicated that the effects of LFS upon the fully-kindled brain were primarily "convulso-suppressive", as under normal circumstances a convulsive seizure would be reliably triggered by applying an ADT-equivalent stimulus. In this case, following LFS administration, some animals had a discharge but no recruitment of neurons to establish the stage 5. Further evidence for the ability of LFS to suppress seizure activity stems from the fact that both strains exhibited ADTs increases that were too high to measure and in 2 FAST and 2 SLOW animals the thresholds remained that high. 
Figure 20 - Average ( \pm S.E.M.) percent changes in the ADTs with LFS $(1 \mathrm{~Hz} ; 300 \mu \mathrm{A} ; 300 \mathrm{~s})$ applied to right amygdala electrode with ADT tested on the kindled amygdala (Note the increments on the Y-axis scale).

('*” = significantly higher ADTs than baseline, $\mathrm{p}<0.001)$ 


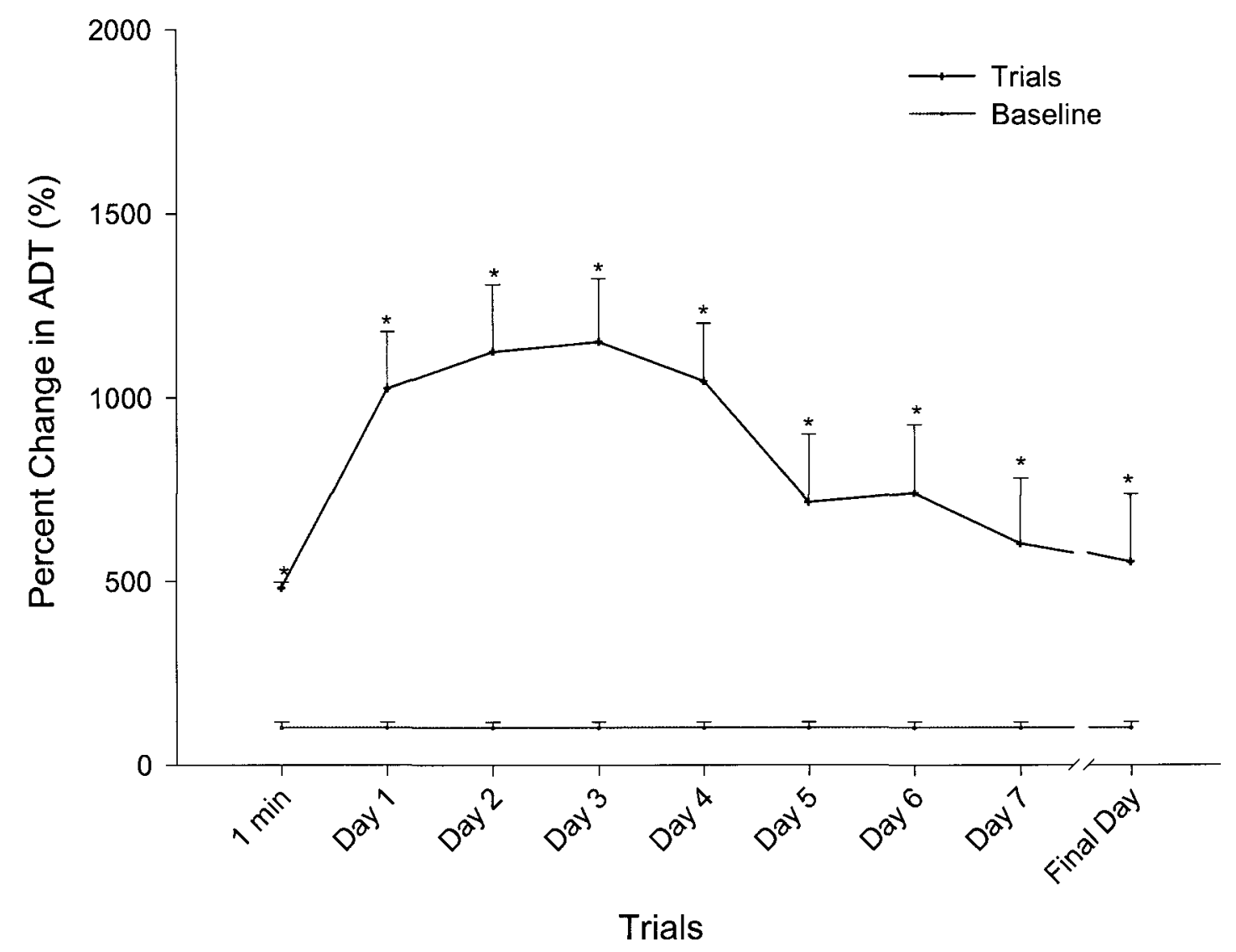


2) LFS administration to the non-kindled spanning electrode with ADT tested on the kindled right amygdala

Considering that there were no strain effects in this condition, data were collapsed over trials revealing a significant effect of percent change in the ADT over trials $(p<0.006)$. Within one minute of the LFS treatment ADTs rose by $257.1 \pm 111.6 \%$ above baseline values with a maximum change occurring on Day 5 with a $512.4 \pm 109.0 \%$ change from the baseline value. Also, the thresholds did not return to baseline but stabilized at $236.0 \pm$ $124.00 \%$ above baseline (Figure 21).

Due to the small sample of the experimental subjects in this treatment condition, the variability in the data was substantial. Despite this both strains, $40 \%$ of the FAST and $50 \%$ of the SLOW rats, exhibited spontaneous seizure activity. The comparison of the two strains showed that the "convulso suppressive" effect of LFS was more frequently observed $(>10 \%)$ in the FAST than in the SLOW group (Table 1). 
Figure 21 - Average ( \pm S.E.M.) percent changes in ADT with LFS $(1 \mathrm{~Hz} ; 300 \mu \mathrm{A} ; 300 \mathrm{~s})$ applied to non-kindled spanning electrode with ADT tested on the kindled amygdala (Note large Y-axis scale).

( **" = significantly higher ADTs than baseline, $\mathrm{p}<0.001)$ 


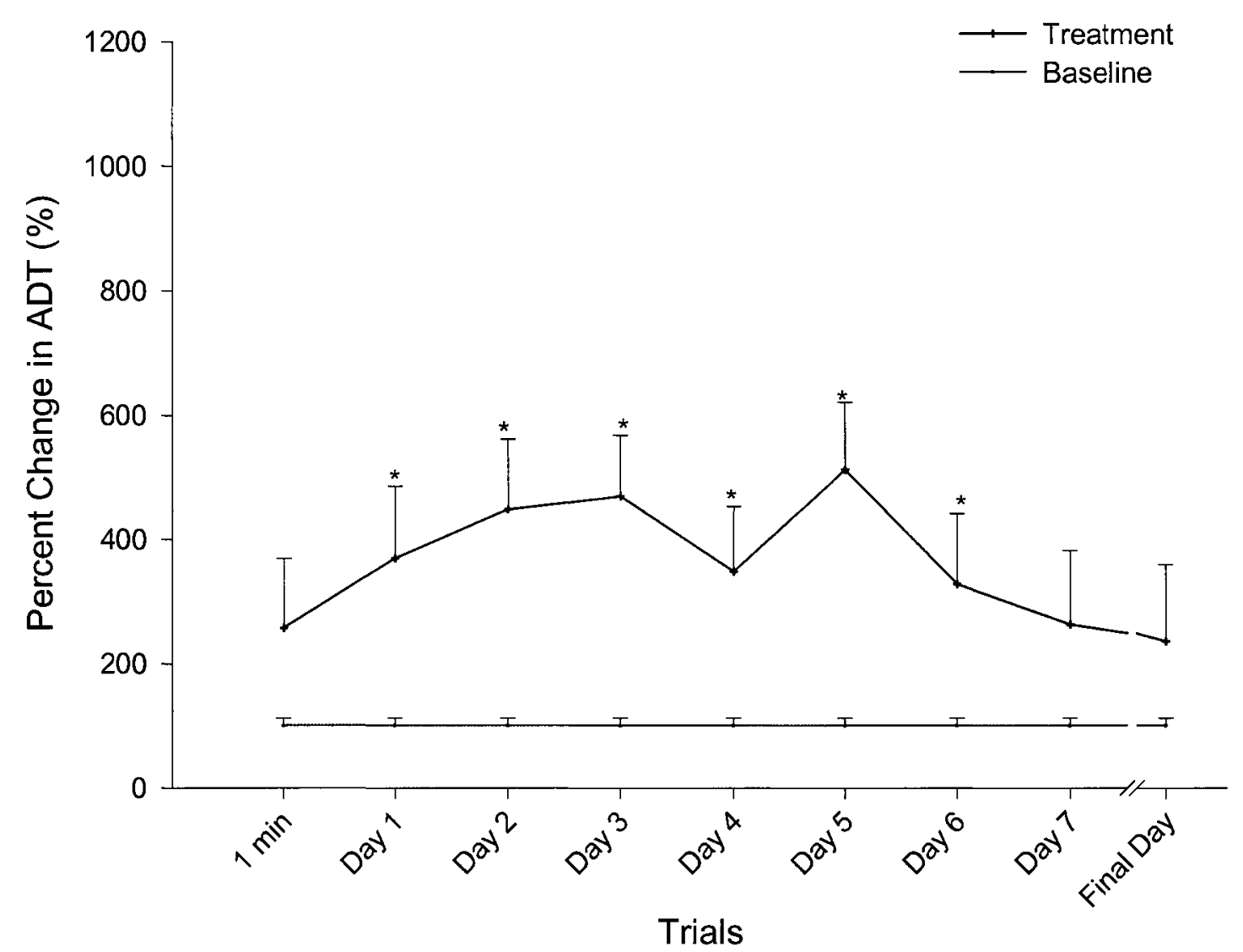


TABLE 1 - Average ( \pm S.E.M.) percent of Fast and Slow rats that experienced spontaneous seizures, seizure failures and immeasurable ADTs after the application of LFS on the kindled amygdala and spanning electrode with ADTs tested on the kindled amygdala. 


\begin{tabular}{|c|c|c|c|c|}
\hline & \multicolumn{2}{|c|}{$\begin{array}{l}\text { LFS administered to kindled } \\
\text { amygdala electrode with ADT } \\
\text { tested on kindled amygdala }\end{array}$} & \multicolumn{2}{|c|}{$\begin{array}{l}\text { LFS administered to spanning } \\
\text { electrode with ADT tested on } \\
\text { kindled amygdala }\end{array}$} \\
\hline & $\operatorname{Fast}(\mathrm{N}=6)$ & Slow $(\mathrm{N}=6)$ & Fast $(\mathrm{N}=5)$ & Slow $(\mathrm{N}=2)$ \\
\hline $\begin{array}{l}\text { Spontaneous } \\
\text { seizures }(\%)\end{array}$ & $\begin{array}{l}33.33 \\
(2 / 6)\end{array}$ & $\begin{array}{l}16.67 \\
(1 / 6)\end{array}$ & $\begin{array}{l}40.00 \\
(2 / 5)\end{array}$ & $\begin{array}{l}50.00 \\
(1 / 2)\end{array}$ \\
\hline $\begin{array}{l}\text { Seizure Failure } \\
(\%)\end{array}$ & $13.22 \pm 7.71$ & $1.58 \pm 0.56$ & $10.37 \pm 3.88$ & $2.08 \pm 1.13$ \\
\hline $\begin{array}{l}\text { Rats with seizure } \\
\text { Failure (\%) }\end{array}$ & $\begin{array}{l}83.33 \\
(\mathrm{~N}=5 / 6)\end{array}$ & $\begin{array}{l}16.76 \\
(N=1 / 6)\end{array}$ & $\begin{array}{l}60.00 \\
(N=4 / 5)\end{array}$ & $\begin{array}{l}50.00 \\
(\mathrm{~N}=1 / 2)\end{array}$ \\
\hline $\begin{array}{l}\text { Trials with ADTs } \\
>1250 \mu \mathrm{A}(\%)\end{array}$ & $24.24 \pm 7.67$ & $41.76 \pm 12.15$ & $\begin{array}{l}21.59 \pm \\
6.80\end{array}$ & $42.87 \pm 2.129$ \\
\hline $\begin{array}{l}\text { Rats with ADTs } \\
>1250 \mu \mathrm{A}(\%)\end{array}$ & $\begin{array}{l}83.33 \\
(\mathrm{~N}=5 / 6)\end{array}$ & $\begin{array}{l}83.33 \\
(\mathrm{~N}=5 / 6)\end{array}$ & $\begin{array}{l}60.00 \\
(\mathrm{~N}=3 / 5)\end{array}$ & $\begin{array}{l}100.00 \\
(\mathrm{~N}=2 / 2)\end{array}$ \\
\hline $\begin{array}{l}\text { Rats with } \\
\text { spindles, } \\
\text { discharges }(\%)\end{array}$ & $\begin{array}{l}100.00 \\
(N=6 / 6)\end{array}$ & $\begin{array}{l}100.00 \\
(\mathrm{~N}=6 / 6)\end{array}$ & $\begin{array}{l}100.00 \\
(\mathrm{~N}=5 / 5)\end{array}$ & $\begin{array}{l}100.00 \\
(\mathrm{~N}=2 / 2)\end{array}$ \\
\hline
\end{tabular}




\section{Histology}

Histological analysis established that the electrodes were placed within the specified regions. Further investigations with the use of cresyl violet and fluorojade B staining showed no neuronal damage or lesioning outside the area of the electrode tip. 


\section{EXPERIMENT 5: KINDLED BRAIN AND THE "MILD-STATUS" EFFECT}

Studies have shown that the induction of a self-sustaining status epilepticus through prolonged electrical stimulation of the amygdaloid region is possible (McIntyre et al., 1982; Racine et al., 1973a). Furthermore, Brandt and associates (2003) showed, using their experimental protocol of electrically-induced status epilepticus, that spontaneous seizure activity can be achieved in non-kindled animals. Thus far, in the present study kindling was involved in all of the previous experiments. However, it is unclear whether the kindled state was a necessary prerequisite for the mild-status effect, which was observed in experiments 3 and 4. The present experiment was, therefore, designed to determine whether LFS-induced spontaneous seizures would occur in a nonkindled brain.

\section{Animals:}

Only naïve Fast rats were used for this investigation with the animals being split into two groups - Kindled $(n=4)$ and Non-kindled $(n=4)$. Due to the loss of headplug assemblies the non-kindled sample was reduced to only 2 animals.

\section{Surgery and Kindling}

The surgical protocol and electrode implantation was identical to that in the Experiment 3. That is, all animals were implanted with a discrete electrode in the right amygdala region and with a spanning electrode in the right hemisphere (see Figure 14 and Appendix 1). The kindled group received stimulations on the discrete electrode 
located in the right amygdala. During kindling, the non-kindled group was exposed to the kindling procedure, but without actually being stimulated.

\section{Histology}

Once experimentation was completed the animals were sacrificed and histological staining was done with cresyl violet and fluorojade B to affirm electrode placement and any possible neuropathology as a result of electrode insertion and/or experimental manipulation.

\section{LOW FREQUENCY STIMULATION (LFS) MANIPULATIONS}

Both experimental groups were administered LFS $(1 \mathrm{~Hz})$ at $300 \mu \mathrm{A}$ for $300 \mathrm{sec}$ on the spanning electrode, with subsequent ADTs recorded from the discrete electrode located in the right amygdala one minute and daily thereafter.

\section{RESULTS}

For this treatment no interaction or trial effects reached significance. However, spontaneous seizures occurred in $75 \%$ of the Kindled and $50 \%$ of the Non-kindled rats (Table 2). 
TABLE 2 - Average ( \pm S.E.M.) percent of Kindled and Non-kindled rats that experienced spontaneous seizures and seizure failures. 
Kindled

Spontaneous Seizures $(\%) \quad 75.00$

$(\mathrm{N}=3 / 4)$

Seizure Failures (\%)
75.00

$(\mathrm{N}=3 / 4)$
Non-kindled

50.00

$\mathrm{N}=(1 / 2)$

100.00

$(\mathrm{N}=2 / 2)$ 


\section{DISCUSSION}

The purpose of the study was to investigate whether LFS would produce antiepileptic effects against fully-kindled seizures in two strains, one genetically seizure-prone (FAST) and the seizure-resistant (SLOW). Although previous investigations studying possible seizure suppressive property of LFS showed a positive effect in altering seizure behavior, the findings were greatly confounded by poor experimental design and shortcomings in the presentations of the data. Therefore, it was not possible to obtain a definitive conclusion concerning the question at hand.

As a result, the present study was novel in a sense that it attempted to address the question in a more systematic and experimentally controlled manner. More specifically, the study consisted of 5 individually performed and logically related experiments to assess the effects of LFS upon ADTs in two rat strains with inherent differences in seizure disposition, through the manipulation of a number of experimental factors, such as anatomical locale of stimulating and recording electrodes, electrode-specific properties, LFS properties (i.e. frequency, intensity and duration), its temporal and spatial parameters, as well as pre-LFS state of the brain (i.e., kindled vs. non-kindled).

The results of the earlier investigators (Gaito et al.,1980; Weiss et al., 1995) of the effects LFS upon seizure activity were limited by the flaws in the experimental design, such as lack of controls, use of stimulation parameters that were commonly associated with necrosis (e.g., d.c. stimulation), confusing manipulation of various experimental parameters of LFS, poor presentation of the results (i.e., confounds of the experimental paradigm) and absence of histological analyses, all of which undermined the veracity of the experimenters' conclusions. 
One of the most salient features of Experiment 1 was the usage of two electrode configurations - traditional twisted, discrete and novel spanning, both located ipsilaterally. The discrete electrode was designed to deliver electrical stimulations to a very circumscribed region of the amygdala, which has been found to be highly susceptible to the kindling effect. The spanning electrode, on the other hand, was used to cover a larger area of the amygdalapiriform complex. The application of the spanning electrode stemmed from the recognition that in human temporal lobe epilepsy, the focus of seizure activity is often unknown and, therefore, an electrical treatment approach would have to involve electrical stimulation of a spanning area of the brain from where the epileptogenic activity is suspected to originate. Both strains of rats were used in Experiment 1.

The purpose of this experiment was to test the previously hypothesized ADTelevating effects of LFS, which has been rationalized to be the possible mechanism of seizure suppression in fully-kindled animals. Our findings showed significant elevations in the ADTs measured in percent change relative to the baseline values, which were established at the end of the kindling protocol. These LFS effects were observed when the stimulations were applied to the previously kindled electrode, regardless of the type of the electrode and when the ADTs were recorded from the same electrode. In contrast, there was no significant LFS effect upon the ADTs when the LFS was administered to the non-kindled/kindled spanning electrode and with the ensuing ADT measurements were obtained from the adjacent kindled, discrete electrode. Thus, clearly LFS applied to the kindled electrode itself was capable of dramatically elevated thresholds for several days - a very antiepileptic effect, but not when the LFS was applied to the adjacent spanning electrode. 
At first sight these results may appear somewhat contradictory. However, there were a number of possible explanations for the findings. The lack of or reduced LFS effect upon the ADTs when the stimulation was given to the spanning electrode, regardless of whether it was kindled or not, might have been due to the following: First, the current strength from the spanning electrode might have had a smaller stimulatory effect upon the nervous tissue by virtue of it being less concentrated than the current applied to the target kindled area through a discrete electrode. Second, the strength of the LFS current across the spanning area might have been dissipated, as it flowed from the small, exposed stimulating electrode tips in the general region of the amygdala where the discrete electrode was located. Thus its impact on the discrete electrode's local network would by minimal. Third, the construction of the spanning electrode might have rendered it less effective at delivering LFS current at its full strength. That is, because it consisted of two pieces of wire with one being directly connected to the source of stimulation and the other being wrapped around it simply for structural integrity without connection to the stimulation source, it might have absorbed (like a sink) some of the current reaching the brain tissue. Fourth, the sheer number of the electroconductive items implanted in and/or over the brain (i.e. the electrodes, jewelers screws) might have further dampened the strength of the LFS stimulus from the spanning electrode in addition to those previously mentioned. Lastly, the effects of LFS through the spanning electrode might have been altered in an as yet unknown manner due to previous electrical manipulations through the discrete electrode.

Despite some limitations, these increases in the ADTs were remarkable given the fact that the post-kindling ADTs, as was shown in ours and previously conducted investigations of the kindling effect (Racine, 1972a, b; Goddard et al., 1969), are normally highly stable. 
Our findings also demonstrated that the increases in the thresholds were significantly greater for the longer duration of the LFS $(90 \mathrm{sec})$. This meant that the stimulus duration might have been an essential stimulation parameter necessary for producing the ADT elevating effects of LFS.

Although, there was no statistically significant strain effect, the SLOW rats did show greater elevations in the ADTs, relative to the FAST across the trials. Furthermore, although the FAST rats' ADTs ultimately returned to their baseline values, the ADTs of the SLOW rats remained above their baseline values. It is conceivable that the lack of strain effect in the first experiment was due to the small sample sizes and the excessive pre-LFS kindling, which otherwise masked pre-existing strain differences.

In the second experiment we followed up on the significant findings of the first experiment, while at the same time attempting to reduce and/or eliminate some of the confounding effects inherent to that experiment. This study controlled for the potential confounding effects of the electrical manipulations performed through the kindled discrete electrode upon those that were administered through the kindled spanning electrode in the same amygdala by now using two experimental groups - one with a spanning electrode and one with a discrete electrode in different amygdalae, i.e., the rats in each condition were implanted with unilateral electrodes to reduce or eliminate any possible dampening effect on the strength of the LFS stimulus by the bilateral electrode arrangement in experiment 1 . By so doing we hoped to obtain more definitive results of LFS upon the ADTs administered through the two types of electrodes. We also attempted to further investigate the possibility of strain differences as the previous experiment did not generate conclusive evidence to that effect. 
Our findings further substantiated the ADT-elevating effect of LFS when the stimulations were applied to the previously kindled electrode, regardless of the type of the electrode, and when the ADTs were recorded from the same electrode. Also, the pattern in which no effects of LFS upon the thresholds were obtained using the non-kindled electrode with the ADTs recorded from the kindled electrode held true. This implied that it was unlikely that the reduced effects of LFS through the spanning electrodes obtained in Experiment 1 were due to the possible stimulatory limitations of the spanning current, the dissipation of the current at distal regions of the brain from the points of stimulation, destructive interference effects of the converging sine current waves, the stimulus strength dampening due to electrode construction and/or the electroconductive properties of the electrodes and the related hardware. Therefore, it appeared that the likely determining variable of the effects of LFS upon the elevation of the ADTs was the kindled state of the brain.

In relation to the question of strain differences, the results were less clear. A small, yet statistically significant strain effect was obtained with $30 \mathrm{sec}$ LFS stimulation only when it was administered through the discrete electrode inserted in the previously kindled amygdala. No such strain differences were seen in the $90 \mathrm{sec}$ treatment, regardless of the electrode type.

A possible explanation for these findings can be made in light of the fact that the FAST rats usually have lower ADTs than the SLOW rats post-kindling (Racine et al., 1999; McIntyre et al., 1999), and therefore a longer LFS stimulation might be required to generate substantial ADT changes in the FAST as compared with the SLOW rats. The fact that this difference was not observed when LFS stimulation was administered to the previously 
kindled spanning electrode may be due to the trade off between stimulation duration and the surface area that was exposed to the stimulation. That is, by stimulating a greater area of the brain, even if the stimulation duration is shorter, it is possible that a sufficiently large area of the targeted amygdaloid-piriform region is recruited by LFS to produce increases in the ADT. This would result in it being resistant enough to the subsequently high frequency stimulations used to test for the ADTs, which would otherwise bring down the ADTs to the baseline value if LFS was administered through the discrete electrode.

However, this explanation for the seemingly effective property of the spanning electrode in eliciting ADT increases in both strains regardless of stimulation duration must be further evaluated against the magnitude of the percent changes that were obtained when LFS was administered though the discrete electrode. Namely, this condition produced substantially higher percent changes in ADT in the SLOW rat and even a small effect on the ADTs of the FAST rat was substantial in the context of the post-kindling ADT being highly stable.

The effectiveness of the discrete electrode in producing large increases in the magnitude of the $\mathrm{ADT}$ was further substantiated when the $90 \mathrm{sec}$ treatment was compared to that obtained through the spanning electrode whose ADT elevations, although statistically significant, were much lower. Therefore, taking all the factors into consideration, it appears that the discrete electrode is a better choice for the elicitation of ADT elevations. On the other hand, to apply LFS as a treatment in the human condition of TLE, it would be desirable to have produced a clearer picture of seizure threshold elevation using the spanning electrode. 
The fact that $90 \mathrm{sec}$ LFS produced percent change increases in the ADTs in both strains and that these changes were significantly greater than in the $30 \mathrm{sec}$ condition, further supported our assumption made in the first experiment that stimulus duration was an essential stimulation parameter necessary for the ADT-elevating effects of LFS.

Thus far, the application of LFS produced a much greater elevation on the kindled discrete electrode than on the kindled spanning electrode. LFS of longer durations also produced better ADTs elevations. A possible reason for the modest effect observed on the spanning electrode was that maybe the small area exposed at the tip of the spanning electrode was not a sufficiently large enough area for the LFS current to express its effects. Therefore by increasing the surface area of the spanning electrode tip, we hoped to increase the magnitude of the LFS effect through the spanning electrode, as in experiment 3 . Using a modified spanning electrode with very exposed tips, we first re-established that LFS applied to the usual kindled discrete electrode with ADT tested on this kindled electrode resulted in a significant elevation in thresholds as seen in our earlier experiments. Then we tested the effects of LFS applied to the modified spanning electrode on the ADTs of the kindled discrete electrode. With application of the LFS on the spanning electrode and ADTs tested on the kindled discrete electrode, we again got modest effects in our ADT measures. However, considering that we calculated the baseline values and subsequent changes in the ADT in this treatment from the last stable thresholds found in the previous treatment where there were great increases in ADTs, which remained well above baseline, did we as a consequence reduce the LFS effect via the spanning electrode? Namely, in using this previously elevated value as the new baseline for the later experiment was a spanning effect masked? The small effect found in using the spanning electrode to delivery the LFS could have been a result of 
the already elevated thresholds from the previous conditions. None of these effects however, in our various experimental paradigms with LFS were associated with any neuropathology, unlike other studies in which lesions were caused by the direct currents (DC) used to administer the LFS. (Weiss et al., 1998)

So far, our results indicate that both the kindled site and duration of the LFS plays a prominent role in the rise of the ADT. The intensity was only now being tested in experiment 3, but the effects of increasing the intensity of the LFS applied to the spanning electrode and tested on the discrete did not appear to cause any significant changes in the subsequent ADTs. However, manipulating both the intensity and duration of LFS applied to the spanning electrode with ADTs tested on the discrete electrode produced extreme elevations in the ADTs beginning 1 minute after the stimulation and remaining elevated for a long duration of time. This combination was of great importance, as ADTs measured on the discrete when the LFS was administered on the spanning, have so far, yielded only modest results.

Studies have shown the elevation of seizure thresholds by increasing the LFS duration (Gaito, 1980) and we have also already addressed the efficacy of long duration LFSs on the elevations in ADT, but the changes seen in experiment 3 were unprecedented. We can only surmise that by altering both the stimulus intensity and duration somehow facilitated these large changes in the ADT of the kindled discrete electrode by way of LFS applied to the spanning electrode. However, these large elevations in ADT beginning within one minute of the ADT also had associated with them the exhibition of spontaneous recurrent seizures.

Consequently it was very important to recognize that the ADTs likely increased at the kindled discrete electrode because that LFS applied for 5 minutes just one minute earlier set the stage for discrete electrode to trigger a mild form of status epilepticus (a state of 
continuous or intermittent seizure activity lasting for 30 minutes or more without regaining consciousness; Fountain, 2000). The subsequent days of elevated thresholds were possibly a reflection of the consequence of developing status epilepticus, and might simply reflect significant neurodegeneration within the limbic system. Experimental studies have shown that prolonged status epilepticus could result in considerable cell loss in the CA1 and CA3 region of the hippocampus and the piriform cortex (Heinemann, 2004; Sutula and Pitkanen, 2003). However, both histological stains (cresyl violet and fluoro-jade B) that were carried out that would expose such pathology indicated no necrotic cells in any of these areas. Thus, clear evidence of brain damage associated with status epilepticus (SE) was absent.

Hence, we have in effect generated a non-pathological form of SE by an electrical stimulation. Previous studies have shown that low to high intensity electrical stimulations $(400-1000 \mu \mathrm{A})$, at high frequencies $(60 \mathrm{~Hz})$, administered continuously or in pulses for a protracted period of time (25-60 mins) to a kindled focus can result in the development of SE (Brandt et al., 2003; Hanforth \& Treiman, 1994; McIntyre et al., 1982). Nonetheless, we achieved a SE-like effect by using a low frequency $(1 \mathrm{~Hz})$ and moderate intensity $(300 \mu \mathrm{A})$, but without the associated neuropathology. Therefore, in using LFS therapeutically or clinically, one should be extremely careful about the parameters of the LFS, as a parametric change could result in alteration to the neural network producing some form of SE. Thus, within limits, LFS can have an anti-epileptic effect of elevating seizure thresholds, yet it also has the potential, with alterations of the parameters of the stimulus, of triggering a protracted seizure, not just a single discrete seizure, but status epilepticus.

Also in conjunction with the generation of a mild form of SE, another effect produced was the high incidence of failed convulsive seizures during the ADT tests. Could it be that 
this raising the threshold also dampened the recruiting power of the neuronal network? This would be an area that needs to be further studied. Conceivably, the SE effect that we created may have been due in a large part to the many previous stimulation procedures that were applied to the spanning electrode before the SE effect appeared. However, in experiment 4, we showed that with minimal previous stimulation experience, LFS for 5 minutes, created heightened elevations in ADTs similar to those in experiment 3 as well as setting the stage for the generation of spontaneous seizures in some of the animals.

We don't know what neurological condition is provided by the LFS to facilitate the appearance of spontaneous seizures; certainly the discrete kindled seizure triggers it, but some previous change in activity must encourage the synchrony necessary to provoke SE. One hypothesis that we tested was whether the initial kindling at the discrete electrode primed the neural network to be more susceptible for this LFS sensitive SE effect. Other works have shown that kindling is not necessary to generate stimulation-based SE, as SE can be triggered from the amygdala or piriform cortex with high-frequency electrical stimulation in normal rats (Brandt et al., 2003; Handforth and Treiman, 1994), although kindled tissue is more sensitive (McIntyre et al., 1982). This was verified in experiment 5 where we generated the SE effect in both kindled and non-kindled groups. Thus kindling is not absolutely necessary to show either the LFS threshold elevation effects or the development of SE. 


\section{CONCLUSION:}

The many adverse effects associated with the use of these pharmacologic agents and their inability to treat certain forms of intractable, pharmacoresistant epilepsy, which creates a need to further investigate non-pharmacological treatment options with little or no adverse effects observed in the patient. Current therapeutic use of the old technique of electrical stimulation is actively being explored. The findings of this study indicated that the use of LFS has the potential to be incorporated into the catalog of therapeutic procedures for the treatment of temporal lobe epilepsy, especially in the case of intractability. This anticonvulsant effect occurs mainly because LFS elevates the ADT, but further can suppress seizure spread and truncate convulsion expression. Paradoxically, however, LFS can set the stage for triggering a mild form of SE. This is further associated with vastly elevated ADTs, the significance or mechanism of which is yet unknown. It might be based on long-term depression (LTD) which can be induced by low frequency stimulation (Mutoh et al., 2005), but further studies are necessary to test this hypothesis. 


\section{APPENDIX 1 - TYPE AND LOCATION OF IMPLANTED ELECTRODES USING BREGMA AS A REFERENCE FOR ALL EXPERIMENTAL PROTOCOLS}

\begin{tabular}{|c|c|c|c|c|}
\hline Electrode & Hemisphere & $\begin{array}{l}\text { Anterior } \\
(\mathrm{mm})\end{array}$ & $\begin{array}{l}\text { Posterior } \\
(\mathrm{mm})\end{array}$ & $\begin{array}{l}\text { Lateral } \\
(\mathrm{mm})\end{array}$ \\
\hline
\end{tabular}

\begin{tabular}{|c|c|c|c|c|c|c|}
\hline $\begin{array}{l}\text { All } \\
\text { Expts. }\end{array}$ & Ground & Right & 10.0 & - & 2.0 & On dura \\
\hline \multirow[t]{3}{*}{$\begin{array}{l}\text { Expt } \\
1\end{array}$} & Amygdala & $\begin{array}{l}\text { Left } \\
\text { Right }\end{array}$ & $\begin{array}{l}0.0 \\
0.0\end{array}$ & $\begin{array}{l}0.0 \\
0.0\end{array}$ & $\begin{array}{l}4.5 \\
4.5\end{array}$ & $\begin{array}{l}8.5 \\
8.5\end{array}$ \\
\hline & $\begin{array}{l}\text { Spanning } \\
\text { Electrode } \\
\text { Unit } 1\end{array}$ & $\begin{array}{l}\text { Left } \\
\text { Right }\end{array}$ & $\begin{array}{l}2.0 \\
2.0\end{array}$ & - & $\begin{array}{l}4.5 \\
2.0\end{array}$ & $\begin{array}{l}8.0 \\
8.0\end{array}$ \\
\hline & $\begin{array}{l}\text { Spanning } \\
\text { Electrode } \\
\text { Unit } 2\end{array}$ & $\begin{array}{l}\text { Left } \\
\text { Right }\end{array}$ & - & $\begin{array}{l}2.0 \\
2.0\end{array}$ & $\begin{array}{l}4.5 \\
6.5\end{array}$ & $\begin{array}{l}9.0 \\
9.0\end{array}$ \\
\hline \multirow[t]{3}{*}{$\begin{array}{l}\text { Expt } \\
2\end{array}$} & Amygdala & Right & 0.0 & 0.0 & 4.5 & 8.5 \\
\hline & $\begin{array}{l}\text { Spanning } \\
\text { Electrode } \\
\text { Unit } 1\end{array}$ & Left & 2.0 & - & 4.5 & 8.0 \\
\hline & $\begin{array}{l}\text { Spanning } \\
\text { Electrode } \\
\text { Unit } 2\end{array}$ & Left & - & 2.0 & 4.5 & 9.0 \\
\hline \multirow[t]{3}{*}{$\begin{array}{l}\text { Expt } \\
3,45\end{array}$} & Amygdala & Right & 0.0 & 0.0 & 4.5 & 8.5 \\
\hline & $\begin{array}{l}\text { Spanning } \\
\text { Electrode } \\
\text { Unit } 1\end{array}$ & Right & 2.0 & - & 4.5 & 8.0 \\
\hline & $\begin{array}{l}\text { Spanning } \\
\text { Electrode } \\
\text { Unit } 2\end{array}$ & Right & - & 2.0 & 4.5 & 9.0 \\
\hline
\end{tabular}

* Depth of electrode was measured from the dura surface 


\section{REFERENCES:}

Albright, P.S. and Burnham, W.M. (1980). Development of a new pharmacological seizure model: effects of anticonvulsants on cortical- and amygdala-kindled seizures in the rat. Epilepsia. 21: $681-689$.

Ben-Ari, Y. and Cossart, R. (2000). Kainate, a double agent that generates seizures: two decades of progress. Trends in Neurosciences. 23: $580-567$.

Benbadis, S.R. and Tatum, W.O. (2001). Advances in the treatment of epilepsy. American Family Physician. 64:91 - 98.

Boon, P., Vonck, K., Reuck, J. De, Caemaert, J. (2001).Vagus nerve stimulation for refractory epilepsy. Seizure. 10(6):448 - 455

Brandt, C., Glien, M., Potschka, H., Volk, H., Löscher, W. (2003). Epileptogenesis and neuropathology after different types of status epilepticus induced by prolonged electrical stimulation of the basolateral amygdala in rats. Epilepsy Research. 55: 83-103.

Burchfiel J.L., Applegate C.D. (1989). Stepwise progression of kindling: perspectives from the kindling antagonism model. Neuroscience Biobehavioural Review. 13(4):28999

Chavel, S. M., Westerveld, M., Spencer, S. (2003). Long-term outcome of vagus nerve stimulation for refractory partial epilepsy. Epilepsy and Behavior. 4(3): $302-309$

Coulter, D.A. and DeLorenzo, R.J. Basic mechanisms of status epilepticus. Advances in Neurology. 79: $725-33$.

Coulter, D.A., McIntyre, D.C., Löscher, W. (2002). Animal Models of Limbic Epilepsies: What can they tell us? Brain Pathology. 12: $240-256$.

Delgado, J.M.R. and Sevillano, M. (1961). Evolution of repeated hippocampal seizures in the cat. Electroencephalography and Clinical Neurophysiology. 13: $722-727$.

Dreifuss, F. E. (1997). Classification of Epileptic Seizures. In Epilepsy: A Comprehensive Textbook. Philadelphia: Lippincott-Raven Publishers. (ed. J. Engel and T.A. Pedley), pp $517-524$.

Durand, D.M. and Bikson, M. (2001). Suppression and control of epileptiform activity by electrical stimulation: A review. Proceedings of the IEEE. 89(7):1065 - 1082.

Fanselow, E.E., Reid, A.P., Nicolelis, M.A.L. (2000). Reduction of pentylenetetrazoleinduced seizure activity in awake rats by seizure-triggered trigeminal nerve stimulation. Journal of Neuroscience. 20(21): $8160-8168$ 
Fisher, R.S. and Handforth, A. (1999). Reassessment: Vagus nerve stimulation for epilepsy. A report of the therapeutics and technology assessment subcommittee of the American Academy of neurology. Neurology. 53: $666-669$

Fisher, R.S., van Emde Boas, W., Blume, W., Elger, C., Genton, P., Lee, P., Engel Jr., J. (2005). Epileptic seizures and epilepsy: Definitions proposed by the International League Against Epilepsy (ILAE) and the International Bureau fro Epilepsy (IBE). Epilepsia. 46(4): $470-471$.

Freitas, R.M., Sousa, F.C, Vasconcelos, S.M., Viana, G.S., Fonteles, M.M. (2004). Pilocarpine-induced status epilepticus in rats: lipid peroxidation level, nitrite formation, GABAergic and glutamatergic receptor alterations in the hippocampus, striatum and frontal cortex. Pharmacology Biochemistry \& Behavior. 78(2):327 - 332 .

Fountain, N.B. Status epilepticus: risk factors and complications. Epilepsia. 41(Suppl 2): $23-30$.

Gaito, J. (1984). Suppression of kindling behavior. Journal of Psychology. 118:113125 .

Gaito, J. (1981). The effect of low frequency and direct current stimulation on the kindling phenomenon in rats. Canadian Journal of Neurological Science. 8(3):249-53.

Gaito, J. (1980a). Gradient of interference by various frequencies on $60 \mathrm{~Hz}$ kindling behavior. Canadian Journal of Neurological Science. (3): 223 - 226.

Gaito, J. (1980b). The effect of variable duration one hertz interference on kindling. Canadian Journal of Neurological Science. 7(1):59 - 64.

Gaito, J. and Gaito, S.T. (1981). The effect of several intertrial intervals on the $1 \mathrm{~Hz}$ interference effect. Canadian Journal of Neurological Science. 8(1):61 - 65.

Gaito, J., Nobrega, J.N., Gaito, S.T. (1980). Interference effect of $3 \mathrm{~Hz}$ brain stimulation on kindling behavior induced by $60 \mathrm{~Hz}$ stimulation. Epilepsia. 21(1): $73-84$.

Goddard, G., McIntyre, D. C., Leech, C. (1969). A permanent change in brain function resulting from daily electrical stimulation. Experimental Neurology. 25: $295-330$

Goodman, J.H., Berger, R.E., Tcheng, T.K. (2005). Preemptive low-frequency stimulation decreases the incidence of amgdala-kindled seizures. Epilepsia. 46(1): 1 - 7

Handforth, A. and Treiman, D. M. (1994). A new, non-pharmacologic model of convulsive status epilepticus induced by electrical stimulation:

Behavioral/electroencephalographic observations and response to phenytoin and phenobarbital. Epilepsy Research. 19: 15-25. 
Hauser, W.A. and Kurland, L.T. (1975). The epidemiology of epilepsy in Rochester, Minnesota, 1935 through 1967. Epilepsia. 16(1): $1-66$.

Heinemann U. (2004).Basic mechanisms of partial epilepsies. Current Opinion in Neurology. 17(2):155-9.

Henry T.R., Votaw, JR., Pennell, P.B., Epstein, C.M., Bakay, R.A., Faber, T.L., Grafton, S.T., Hoffman, J.M. (1999). Acute blood flow changes and efficacy of vagus nerve stimulation in partial epilepsy. Neurology. 52(6):1166 - 1173.

Jackson, J.H. (1890).The Lumleian lectures on convulsive seizures. British Medical Journal. 1: 765-771

Jaser, H.H., Ward, A.A., Pope, A. (eds) Basic Mechanisms of the Epilepsies. Boston, Little, Brown, 1969)

Kerrigan, J.F., Litt, B., Fisher,R.S., Cranstoun, S., French, J.A., Blum, D.E., Dichter, M., Shetter, A., Baltuch, G., Jaggi,J. Krone, S., Brodie, M. Rise, M., Graves, N. (2004). Electrical Stimulation of the Anterior Nucleus of the Thalamus for the Treatment of Intractable Epilepsy. 45 (4): $346-354$.

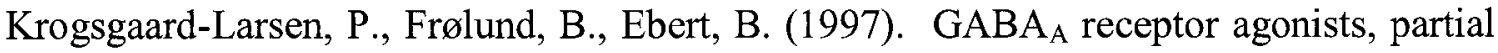
agonists, and antagonists. In: Enna, S. J. and Bowery, N. G. (Eds.) The GABA receptors. Humana Press, New Jersey.

Kullman, D.M. (2005). Genetics of Epilepsy. Journal of Neurology, Neurosurgery and Psychiatry. 73(Suppl II):ii32 - ii35.

Kulkarni, S.K. and George, B. (1994). Kindling Model of Epilepsy. Methods Find Experimental Clinical Pharmacology. 16(10): 735 - 745.

Löscher, W. (1999). Animal models of epilepsy and epileptic seizures. In: Eadie, M.J., Vajda, F. (Eds.), Antiepileptic drugs. Handbook of experimental pharmacology. Springer, Berlin, pp. $19-62$.

Löscher, W. (2002). Current status and future directions in the pharmacotherapy of epilepsy. Trends in Pharmacological Science. 23:113 - 118.

Löscher, W., Czuczwar, S.J., Wolff, G.L. (1986). AE mice: an inbred mouse strain with interesting features for epilepsy research. Epilepsia. 27: 657-664.

Löscher, W., Jäckel, R., Czuczwar, S.J. (1986). Is amygdala kindling in rats a model for drug-resistant partial epilepsy? Experimental Neurology. 93: $211-226$. 
Löscher, W. \& Schmidt, D. (1988). Which animal models should be used in the search for antiepileptic drugs? A proposal based on experimental and clinical considerations. Epilepsy Research. 2: 145-181.

Lothman, E.W., Bertram, E.H., Stringer, J.L. (1991). Functional anatomy of hippocampal seizures. Progress Neurobiology. 37: $1-82$.

Manno, E.M. (2003). New management strategies in the treatment of status epilepticus. Mayo Clinic Proceedings. 78(4): 508-18.

Maynert, E.W. and Kusek J.C. (1980). Phenobarbital: chemical-biophysical effects. Advances in Neurology. 27: 541 - 52.

McIntyre, D.C. and Goddard, G.V. (1973). Transfer, interference and spontaneous recovery of convulsions kindled from the rat amygdala. Electroencephalography and Clinical Neurophysiology. 35: 533 - 543.

McIntyre, D.C., Nathanson, D., Edson, N. (1982). A new model of partial status epilepticus based on kindling. Brain Research. 250(1): $53-63$.

McIntyre, D.C., Kelly, M.E. Dufresne, C. (1999). FAST and SLOW amygdala kindling rat strains: Comparison of the Amygdala, Hippocampal, piriform and perirhinal cortex kindling. Epilepsy Research. 35: 197-209.

McIntyre, D.C., Poulter, M.O., Gilby, K. (2002). Kindling: some old and some new. Epilepsy Research. 50(1-2):79 - 92.

McNamara, J.O. and Wada, J.A. (1997). Kindling Model. On: Epilepsy: A Comprehensive Textbook. Engel, J. and Pedley, T.A. (Eds.). Lippincott-Raaven Publishers, Philadelphia. pp. $419-425$.

Michalakis, M, Holsinger, D, Ikeda-Douglas, C., Cammisuli, S., Ferbinteanu, J., DeSouza, C., DeSouza, S., Fecteau, J., Racine R.J., Milgram N.W. (1998) Development of spontaneous seizures over extended electrical kindling. I. Electrographic, behavioral, and transfer kindling correlates. Brain Research.793 (1-2):197-211.

Mohapel, P. and McIntyre, D,C. (1998). Amygdala kindling-resistant (SLOW) or -prone (FAST) rat strains show differential fear responses. Behavioural Neuroscience. 112(6): 1402-13

Molino, A. and McIntyre, D.C. (1972). Another inexpensive headplug for electrical recording and or stimulation of rats. Physiology and Behaviour. 9: $273-275$.

Morimoto, K., Fahnestock, M., Racine, J. (2004). Kindling and status epilepticus models of epilepsy: rewiring the brain. Progress in Neurobiology. 73: $1-60$. 
Mutoh, H., Yuan, Q., Knöpfel. (2005). Long-Term Depression at Olfactory Nerve Synapses. Journal of Neuroscience. 25(17): 4252-4259.

Nilsson, M.H., Tornqvist, A.L., Rehncrona, S. (2005). Deep-brain stimulation in the subthalamic nuclei improves balance performance in patients with Parkinson's disease, when tested without anti-parkinsonian medication. Acta Neurologica Scandinavica. 111(5):301 - 308.

Paxinos G. and Watson, C. (2005). The Rat Brain in Stereotaxic Cordinates. Elsevier Academic Press, London

Pelligrino, L.J., Pelligrino, A.S., Cushman, A.J. (1979). A stereotaxic atlas of the rat brain. Plenum Press, New York.

Pinel, J.P.J. (1981). Kindling-induced experimental epilepsy in rats: cortical stimulation. Experimental Neurology. 72: $559-569$.

Pitkanen, A. and Sutula, T.P. (2002). Is epilepsy a progressive disorder? Prospects for new therapeutic approaches to temporal lobe epilepsy. Lancet Neurology.(3): 172 - 181.

Poulter M.O., Brown, L.A., Tynan, S, Willick, G., William, R., McIntyre, D.C. (1999). Differential expression of $\alpha_{1}, \alpha_{2}, \alpha_{3}$, and $\alpha_{5}$ GABA $_{\mathrm{A}}$ receptor subunits in seizure-prone and seizure-resistant rat models of temporal lobe epilepsy. Journal of Neuroscience. 19(11): $4654-61$.

Racine, R. (1972a). Modification of seizure activity by electrical stimulation. I: Afterdischarge threshold. Electroencephalography and Clinical Neurophysiology. 32: $269-279$.

Racine, R. (1972b). Modification of seizure activity by electrical stimulation. II: Motor Seizure. Electroencephalography and Clinical Neurophysiology. 32: 281 - 294.

Racine, R., Okujava, V., Chipashvili, S. (1972). Modification of seizure activity by electrical stimulation. III: Mechanisms. Electroencephalography and Clinical Neurophysiology. 32: 295 - 299.

Racine, R. (1991). The Kindling Phenomenon: Epilepsy Model or Memory Model? (and other areas of controversy). In: Memory Mechanisms: A Tribute to G.V. Goddard. (Eds. Abraham, W.C., Corballis, M., White, G.) Erlbaum, N.J, pp. $105-125$.

Racine, R.J., Burnham, W.M., Gartner, J.G., Levitan, D. (1973a) First Trial Motor Seizure Development in Rats Triggered by Amygdaloid stimulation in the Rat. Electroencephalography and Clinical Neurophysiology. 35: 487 - 494.

Racine, R.J., Steingart, M., McIntyre, D.C. (1999). Development of kindling-prone and kindling-resistant rats: selective breeding and electrophysiological studies. Epilepsy Research. 35:183-189. 
Sarkisian, M.R. (2001).Overview of the Current Animal Models for Human Seizure and Epileptic Disorders. Epilepsy and Behaviour. 2:201 - 216.

Sato, M., Racine, R.J., McIntyre, D.C. (1990). Kindling: basic mechanisms and clinical validity. Electroencephalography and Clinical Neurophysiology. 76: $459-472$.

Schwartzkrion, P.A. and Wyler, A.R. (1980). Mechanisms Underlying Epileptiform Burst Discharge. Annals of Neurology. 7: $95-107$.

Scott, R.C., Surtees, R.A., Neville, B.G. (1998). Status epilepticus: pathophysiology, epidemiology, and outcomes. Archives of Disease in Childhood. 79(1): $73-77$.

Seyfried T.N. and Glaser G.H. (1985). A review of mouse mutants as genetic models of epilepsy. Epilepsia. 26(2): $143-50$.

Sperk, G., Lassmann, H., Baran, H., Seitelberger, F., Hornykiewicz, O. (1985) Kainic acid-induced seizures: dose-relationship of behavioural, neurochemical and histopathological changes. Brain Research. 338(2):289 - 95.

Sun, B., DeSalles, A.A.F., Medin, P.M., Solberg, T.D., Hoebel. B., Felder-Allen, M., Krahl, S.E., Ackerman, R.F. (1998). Reduction of Hippocampal-Kindled Seizure Activity in Rats by Stereotactic Radiosurgery. Experimental Neurology. 154:691 - 695.

Sutula T.P., Hagen J., Pitkanen, A. (2003). Do epileptic seizures damage the brain? Current Opinion in Neuroogyl. 16(2):189-95.

Theodore, W.H. and Fisher, R.S. (2004). Brain stimulation for epilepsy. The lancet Neurology. 3: $111-118$.

Treiman, D. (2001). GABAergic Mechanisms in Epilepsy. Eplepsia.42:8 - 12

Tu, B. Timofeeva, O., Jiao, Y., Nadler J.V. (2005). Spontaneous release of neuropeptide $\mathrm{Y}$ tonically inhibits recurrent mossy fiber synaptic transmission in epileptic brain. Journal of Neuroscience. 25: 1718 - 1729.

Velasco, M., Velasco, F., Velasco, A.L. (2001). Centromedian-thalamic and hippocampal electrical stimulation for the control of intractable epileptic seizures. Journal of Clinical Neurophysiology. 18(6): $495-513$.

Velasco ,A.L., Velasco, M., Velasco, F., Menes, D., Gordon, F., Rocha, L., Briones, M., Marquez, I. (2000). Subacute and chronic electrical stimulation of the hippocampus on intractable temporal lobe seizures: preliminary report. Archives of Medical Research. 31(3):316-328. 
Velišek, L., Velíšekova, J., Stanton, P.K. (2002). Low-frequency stimulation of the kindling focus delays basolateral amygdala kindling in immature rats. Neuroscience Letters. 326(1):61 -63.

Vonk, K., Boon, P., Achten, E., de Reuck, J., Caemaert, J. (2002). Long term amygdalohippocampal stimulation for refractory temporal lobe epilepsy. Annals of Neurology. 52: $556-565$.

Wada, J.A. and Osawa, T. (1976). Spontaneous recurrent seizure state induced by daily electrical amygadaloid stimulation in Senegalese baboons (Papio papio). Neurology. 26: $273-286$.

Wada, J.A. and Tsuchimochi, H. (1995). Cingulate kindling in Senegalese baboons, Papio papio. Epilepsia. 36(11):1142 - 1151.

Weiss, S.R.B., Eidsath, A., Li, X, L., Heynen, T., Post. R.M. (1998). Quenching revisited: low level direct current inhibits amygdala-kindled seizures. Experimental Neurology. 154: 185 - 192

Weiss S.R., Li, X.L., Rosen, J.B., Li, H., Heynen, T., Post, R.M. (1995). Quenching: inhibition of development and expression of amygdala kindled seizures with low frequency stimulation. Neuroreport. 6(16):2171 - 2176 . 